https://helda.helsinki.fi

Defect identification in semiconductors with positron annihilation: Experiment and theory

\title{
Tuomisto, Filip
}

2013-11-14

Tuomisto , F \& Makkonen , I 2013 , ' Defect identification in semiconductors with positron annihilation: Experiment and theory ' , Reviews of Modern Physics, vol. 85 , no. 4 , pp. 1583-1631 . https://doi.org/10.1103/RevModPhys.85.1583

http://hdl.handle.net/10138/306582

https://doi.org/10.1103/RevModPhys.85.1583

publishedVersion

Downloaded from Helda, University of Helsinki institutional repository.

This is an electronic reprint of the original article.

This reprint may differ from the original in pagination and typographic detail.

Please cite the original version. 


\title{
Defect identification in semiconductors with positron annihilation: Experiment and theory
}

\author{
Filip Tuomisto* \\ Department of Applied Physics, Aalto University School of Science, Espoo, Finland \\ Ilja Makkonen ${ }^{\dagger}$ \\ COMP Centre of Excellence, Helsinki Institute of Physics and Department of Applied Physics, \\ Aalto University School of Science, Espoo, Finland
}

(published 14 November 2013)

\begin{abstract}
Positron annihilation spectroscopy is particularly suitable for studying vacancy-type defects in semiconductors. Combining state-of-the-art experimental and theoretical methods allows for detailed identification of the defects and their chemical surroundings. Also charge states and defect levels in the band gap are accessible. In this review the main experimental and theoretical analysis techniques are described. The usage of these methods is illustrated through examples in technologically important elemental and compound semiconductors. Future challenges include the analysis of noncrystalline materials and of transient defect-related phenomena.
\end{abstract}

DOI: 10.1103/RevModPhys.85.1583

PACS numbers: 61.72.J-, 78.70.Bj, 71.60.+z, 81.05.-t

\section{CONTENTS}

I. Introduction

A. Defects in semiconductors

1584

1. Role and formation of defects in semiconductors

2. Studying defects in semiconductors

B. Positron annihilation spectroscopy

1. Background

2. Positron annihilation methods

II. Experimental Techniques

A. Positrons in solids

1. Implantation, thermalization, and diffusion

2. Positron states and trapping

3. Trapping model

B. Positron lifetime spectroscopy

1. Experimental details

2. Data analysis

3. Information revealed by the positron lifetime

C. Doppler broadening spectroscopy

1. Experimental details

2. Data analysis

3. Chemical information contained in Doppler spectra

III. Theory and Computational Methods
A. Two-component electron-positron density-functional theory
B. Modeling localized positrons
C. Positron annihilation parameters
1. Annihilation rate and lifetime
2. Momentum density of annihilating electron-positron pairs

\footnotetext{
*filip.tuomisto@aalto.fi

†ilja.makkonen@aalto.fi
}

D. Functionals for electron-positron correlation effects

E. The atomic superposition method

F. Numerical approaches for self-consistent calculations

1603

IV. Results

1605

A. An overview of results obtained in the past two decades

1. Elemental semiconductors $\mathrm{Si}, \mathrm{Ge}$, and $\mathrm{C}$

2. Traditional III-V and II-VI semiconductors

3. Novel semiconductors: III-N, $\mathrm{SiC}$, and $\mathrm{ZnO}$

1607

B. Vacancy-(multi)donor complexes in highly $n$-type doped silicon

1608

C. The vacancy-fluorine complex in silicon and silicon-germanium alloys

D. The EL2 defect in gallium arsenide

E. The gallium vacancy-tellurium complex in gallium arsenide

F. The gallium vacancy and its complexes in gallium nitride

G. Metal vacancy-nitrogen vacancy complexes in III-nitrides and their alloys

$\mathrm{H}$. The substitutional lithium-on-zinc-site defect in zinc oxide

A. Materials with complex crystal structures

1620

C. Positron thermalization and trapping in nanocrystalline, amorphous, and molecular systems

D. Pump-probe experiments with positron annihilation spectroscopy

E. Toward higher slow-positron beam intensity 1623

VI. Summary

References 


\section{INTRODUCTION}

Positron annihilation spectroscopy has been widely used for studying defects in semiconductors since the early 1980s, while the first reports dealing with radiation damage in silicon and germanium had been published already in the 1970s (Cheng and Yeh, 1973; Arifov, Arutyunov, and Ilyasov, 1977). The early developments of both experimental and theoretical approaches applicable to semiconductor studies were reviewed by Schultz and Lynn (1988) and Puska and Nieminen (1994). An introductory book on positron annihilation studies of defects in semiconductors has also been written by Krause-Rehberg and Leipner (1999). Our aim in writing this review is twofold. First, we want to introduce the basic concepts behind the experimental and theoretical methods of positron annihilation and review the latest developments that have led to the possibility of identifying defects in semiconductors with a high level of detail. Second, by going through a variety of examples in both elemental and compound semiconductors, we want to illustrate how these methods can be applied to improve our understanding of the physics of defects in semiconductors.

The organization of this review is as follows. First, we give an introduction to defects in semiconductors and the history and methods of positron annihilation. In the second part, after briefly explaining the necessary concepts related to the behavior of positrons in solids, we delve into the details of the experimental methods most used in semiconductor studies: positron lifetime spectroscopy and Doppler broadening spectroscopy. Here our aim is to give a frank account of the strengths and weaknesses of the experimental setups and analysis methods, hoping to provide useful reference material for the specialist and at the same time provide the nonpractitioner additional means to assess positron results and interpretations. The same approach is applied in the third part where the theoretical methods are presented. In Secs. II and III we go through examples where both experimental and theoretical positron methods have been applied to study various semiconductor materials and defects therein. The focus of these sections is, in addition to showing how the positron methods work in practice, on the interpretations that can be made about the defects identified in these technologically relevant materials. The results are systematically compared to the knowledge obtained by other experimental and theoretical methods in order to give a frame of reference. Finally we discuss the present challenges and possible future directions in semiconductor research with positrons.

It is important to note that we do not attempt to make an exhaustive review of all positron work on defects in semiconductors. To cover most of the published works on positron annihilation in solids, we refer the interested reader to the reviews by Berko and Hereford (1956), Ferrell (1956), Schultz and Lynn (1988), Asoka-Kumar, Lynn, and Welch (1994), Puska and Nieminen (1994), Krause-Rehberg et al. (1998), and Saarinen, Hautojärvi, and Corbel (1998). A number of books have been published on the subject of positron annihilation in solids, as well as chapters in various edited volumes. For detailed accounts see the conference proceedings of the ICPA (International Conferences on Positron Annihilation), SLOPOS (International Workshops on Slow Positron Beams), and PSSD/PSD (Positron Studies on Semiconductors and Defects) and to references therein.

\section{A. Defects in semiconductors}

Lattice defects in semiconductors are like spices in your food: too much is disgusting, too little is worthless, while just the right kind and amount makes the day. Another common feature is that both are typically present in amounts much smaller than the host. There exists a wide variety of review articles and books on defects in semiconductors. For a detailed picture of the field one is strongly advised to browse the proceedings volumes of the ICDS (International Conferences on Defects in Semiconductors). A theoretical perspective can be found in the book by Lannoo and Bourgoin (1981), while a recent volume covers many practical issues easily accessible to the newcomers to the field (McCluskey and Haller, 2012).

\section{Role and formation of defects in semiconductors}

Defects in crystalline solids are static interruptions to the periodicity of the crystal. They can be classified by their spatial extent into point defects that are zero dimensional and extended defects that can be one dimensional (e.g., dislocations), two dimensional (e.g., stacking faults), and three dimensional (e.g., aggregates of impurities). It is not unusual to have important densities of more than one of these kinds of defects in a given crystalline material, such as an elemental (e.g., silicon or germanium) or a compound (e.g., gallium arsenide or zinc oxide) semiconductor. Quite typically they also affect each other's properties and presence, e.g., the formation of stacking faults in a crystal may induce vacancy defects. In this review, the emphasis is on point defects in general and on vacancy defects, in particular, as the positron methods are most sensitive to defects with extra open volume.

In contrast to metals, in semiconductors very dilute concentrations (e.g., less than ppm) of defects may have important effects on the electrical and optical properties. This is due to the electronic states created by the defects in the typically $(0.5-5 \mathrm{eV})$ wide band gap of the semiconductor. Depending on the position in the gap, electrons can be excited to or from these states (from or to the bands or other states in the gap) thermally, electrically, or optically. In practice, the electrical and optical properties of semiconductors are defined by controlled introduction of impurities in the host lattice, but often it is not possible to completely eliminate the formation of other defects, such as vacancy defects, atoms on interstitial lattice sites, extra impurities, or antisite defects (the latter exist only in compound semiconductors). Either these defects have a detrimental effect on the targeted property or sometimes they can assist in obtaining the desired functionality of the material. It is also possible for some defects to be neutral from the point of view of the property to be controlled.

Understanding of the properties of a semiconductor requires (i) identification of the defects present in the lattice, (ii) their quantification, and (iii) knowledge of the nature of the states they introduce in the band gap (i.e., their effects on the properties). Examples of defect properties are subband-gap light absorption and emission, and introduction or removal of electrons to or from the conduction or the valence 
band. Control of the semiconductor properties requires in addition that the formation and introduction mechanisms of these defects are understood, as well as their other physical properties such as how they interact with other defects in the lattice and whether they can be made to move with the hope of them getting trapped at a neutralizing location or driven out of the region of interest. It should not be a surprise that many different experimental and theoretical methods need to be employed in order to obtain even a small part of the required knowledge. Finally, after all this understanding, one needs to be able to manufacture the semiconductor material in such a way that desired defects are introduced but the harmful ones are not. Quite often this is very challenging.

Usually it is rather straightforward to control the introduction of the desired impurities in the semiconductor matrix. Dopants can be added to the growth environment in various ways or they can be diffused in or implanted after the growth process. The most important limitations are solubility in the case of in situ or diffusion doping, while implantation is mostly applicable to close-to-the-surface doping profiles. However, while introduction of dopants is controllable and requires active measures, other kinds of point defects are formed either thermally, due to kinetic or chemical effects, or as radiation damage in the case of implantation processing. In addition, the growth environment may contain some unwanted impurities that are difficult to control: typical omnipresent elements are oxygen and hydrogen. Further, for example, in the case of wide-band-gap semiconductors such as the III-nitrides, where native substrates are not easily available, the lattice mismatch between the thin film and substrate causes initial stresses and strain that are most often relaxed through the generation of dislocations and other extended defects. There are many ways to try to avoid the formation of the unwanted defects or to try to remove them by postprocessing, such as thermal treatments. Even though many defect-related problems have been identified and solved over the past 60 years of semiconductor research, the constant quest for faster, cheaper, less power consuming, and new kinds of electronics generates the need for new materials properties and hence creates new defect-related challenges.

\section{Studying defects in semiconductors}

As the existence of defects is what makes semiconductors such useful materials, defects in semiconductors have been studied for as long as semiconductors have been known. The wide variety of methods can be roughly divided into electrical measurements, optical spectroscopy, particle beam methods, microscopy, and theoretical calculations. Detailed reviews on these methods can be found in the literature [see, e.g., Stavola (1998)]. In the following we briefly go through the defect detection, identification, and quantification capabilities of the most used methods in semiconductor defect studies.

Measuring electrical properties from the defect point of view typically leads to the determination of resistivity (conductivity), free-carrier concentration and mobility, concentrations of ionized donors and acceptors, and deep carrier traps. By definition, these properties can be considered the most basic properties of a semiconductor. The most popular methods employed are Hall effect experiments and deep-level transient spectroscopy (DLTS) [see, e.g., Svensson, Ryden, and Lewerentz (1989), Dobaczewski et al. (1994), and Look, Hemsky, and Sizelove (1999)]. Optical spectroscopies give access to another set of basic properties of semiconductors, namely, the optical absorption and emission that are particularly important in optoelectronic device applications such as light-emitting diodes or laser diodes. Absorption and luminescence spectroscopies provide detailed information on the optical transitions between the valence and conduction bands and on the positions and nature of defect-induced electronic states in the band gap.

The above techniques provide detailed information on the electrical and optical properties generated by the defects, but usually they do not allow for direct identification of the defects in question, and in optical spectroscopy the determination of defect concentrations is challenging (Reshchikov and Morkoç, 2005). Optical absorption by local vibrational modes in the infrared (IR) wavelengths can be used to identify defects through their vibrational frequency fingerprints (Bergman et al., 1988; Gotz et al., 1996). This method is particularly useful in the case of hydrogen-related defects in semiconductors because of the very distinct frequencies originating from the low atomic mass of hydrogen. There is a set of techniques based on photon spectroscopy in the presence of a magnetic field that are very sensitive to the detailed atomic structure thanks to the hyperfine interactions. These methods employ the electron spin resonances (ESRs), and require the defect to be studied to have a paramagnetic ionized state that can be excited by an external field. Variations of these experiments include electron paramagnetic resonance (EPR), optically detected magnetic resonance, and electron nuclear double resonance (Watkins and Corbett, 1964). The ESR methods are sensitive to the number of the active centers (instead of the concentration) and give a very detailed atomic structure of the defects that are detected. Challenges are encountered with samples with high freecarrier concentrations due to efficient microwave absorption, while thin films often have too few active centers in total even if their concentration is high.

The electrical and optical defect spectroscopy methods are intrinsically nondestructive, i.e., the semiconductor samples and their properties are not altered during the measurements. There is a wide variety of methods based on the use of ion beams that in turn are destructive, but provide crucial data on the defect properties. Most common of these are Rutherford backscattering and nuclear reaction analysis which are very efficient for detecting and identifying atoms that are not in correct lattice positions (Wahl et al., 1997; Yu et al., 2002). Nondestructive particle beam methods include muon spin rotation and positron annihilation spectroscopies, of which the first is particularly useful for modeling behavior of hydrogen in semiconductors (Stavola, 1998), while the latter is selectively sensitive to vacancy-type defects. Electron microscopy methods have already reached (sub-)atomic resolution; this holds especially for transmission electron microscopy (TEM). In addition, the latest advances in the so-called $Z$ contrast allow the identification of atomic species as well (Pennycook, 2012). Hence exact positions of atoms can be imaged in sample cross sections, providing direct experimental identification of extended defects and 
impurities, given the fact that the concentrations (densities) are high enough, as the typical size of atomic-resolution images is of the order of $10 \times 10 \mathrm{~nm}^{2}$. Another challenge in imaging intrinsic point defects is that they may be created in the preparation of cross-sectional samples.

Calculations of the electronic structure of semiconductors and their defects is possible from first principles. By far the most popular method is the density-functional theory (supercell calculations) with the electron-electron exchange and correlation described through the local-density approximation (LDA) or semilocal generalized-gradient approximations (GGA). The computing power of modern supercomputers allows for efficient calculations with relatively large supercells (up to 1000 atoms) of the formation enthalpies and charge transition levels of point defects with these methods [for reviews, see Van de Walle and Neugebauer (2004), Drabold and Estreicher (2007), Janotti and Van de Walle (2009), and Van de Walle, Lyons, and Janotti, (2010)]. However, both the LDA and GGA suffer from predicting incorrect band gaps and hence the reliability of the predicted defect levels is often debated. Atomic structures of the defects seem to be less affected by the different approximations. Rather recently so-called hybrid functionals (Becke, 1993; Perdew, Ernzerhof, and Burke, 1996; Adamo and Barone, 1999; Heyd, Scuseria, and Ernzerhof, 2003) have been applied, where part of the exchange and correlation is calculated within the Hartree-Fock approach to improve the description of nonlocal effects. This approximation has significantly improved the predicted band gaps for semiconductors and allowed for new interpretations for some defect levels. At the time of writing this review, the computational complexity of the hybrid functionals limits the supercell sizes to roughly 100 atoms; hence especially in the case of charged defects so-called supercell corrections need to be considered (Makov and Payne, 1995; Schultz, 2000; Freysoldt, Neugebauer, and Van de Walle, 2009). With constantly improving computing power, more accurate approaches, such as the $G W$ quasiparticle approximation and quantum Monte Carlo methods, are becoming more and more applicable in defect calculations [see, e.g., Ertekin et al. (2012) and Rinke et al. (2012)].

\section{B. Positron annihilation spectroscopy}

Positron annihilation spectroscopy is a characterization method for probing the local electron density and atomic structure at the site chosen by the electrostatic interaction of the positron with its environment. The information on the structure can be measured in the time and energy spectra of the positron annihilation radiation. It is thus possible to investigate experimentally local structures embedded in the bulk of the material, such as missing atoms (vacancies), clustering of atoms, superlattices and device structures, quantum dots, as well as free volume, and void sizes in polymers or even biological materials. These imperfections often determine the crucial properties of the materials, such as mechanical properties, electrical conductivity, diffusivity, or light emission. The positron annihilation methods have had a significant impact on defect spectroscopy in solids by introducing an experimental technique for the unambiguous identification of vacancies. Native vacancies have been observed at high concentrations in many semiconductors, and their role in doping and compensation can be quantitatively discussed.

\section{Background}

The existence of the antiparticle of the electron, the positron, was predicted by Dirac (1928), and its first experimental observation came in 1932 (Anderson, 1933). Positronelectron annihilation was eagerly studied throughout the 1940s and 1950s, and experimental methods were developed. In the late 1960s it was understood that positrons were sensitive to lattice defects in metals (MacKenzie et al., 1967; Bergersen and Stott, 1969; Connors and West, 1969; Hodges, 1970). The development of variable-energy slowpositron beams (Schultz and Lynn, 1988) and of the theory of positrons in semiconductors and defects in the 1980s (Puska and Nieminen, 1994) made research on thin films and coatings accessible to positron spectroscopy and led to an ongoing growth in interest in these methods for materials research since the early 1990s (Fig. 1). Positron annihilation spectroscopy is nowadays applied in $\sim 200$ research laboratories worldwide, while there are $\sim 40$ operational slow-positron beams in $\sim 30$ research laboratories.

As described above, many techniques are applied to identify defects in semiconductors on the atomic scale. The advantage of the positron annihilation method lies in its ability to selectively detect vacancy-type defects. This is based on two special properties of the positron: it has a positive charge and it annihilates with electrons. An energetic positron which has penetrated into a solid rapidly loses its energy and then lives for a few hundred picoseconds in thermal equilibrium with the environment. During its thermal motion the positron interacts with defects, which may lead to trapping into a localized state. Thus the final positron annihilation with an electron can happen from various states. Energy and momentum are conserved in the annihilation process, where two photons of about $511 \mathrm{keV}$ are emitted into opposite directions. These photons carry information on the state of the annihilated positron. The positron lifetime is inversely proportional to the electron density encountered by

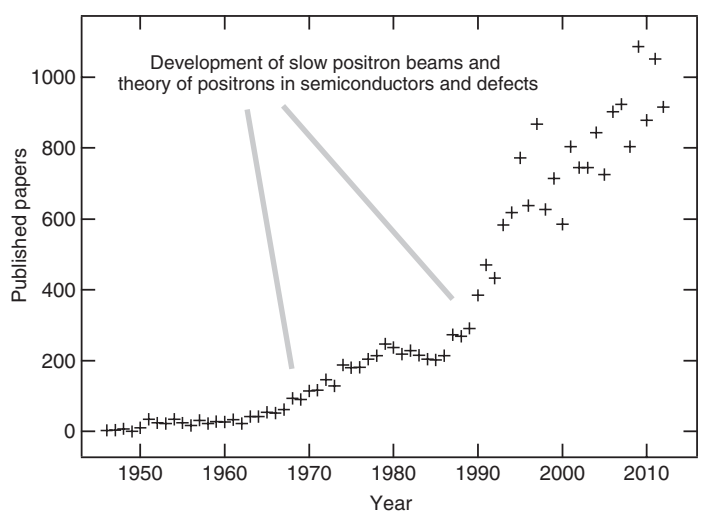

FIG. 1. The number of papers published per year on positrons in condensed matter physics and materials research. The development of slow positron beams and of the theory of positrons in semiconductors and defects during the 1970s and 1980s predates the strong increase in research activity. Data from ISI Web of Science. 
the positron. The momentum of the annihilated electron causes an angular deviation from the $180^{\circ}$ straight angle between the two $511 \mathrm{keV}$ photons and creates a Doppler shift in their energy. Thus the observation of positron annihilation radiation gives experimental information on the electronic and defect structures of solids. For more detailed accounts on the positron annihilation in solids in general, see, e.g., West (1973) and Hautojärvi (1979).

The sensitivity of positron annihilation spectroscopy to vacancy-type defects is easy to understand. The free positron in a crystal lattice experiences strong repulsion from the positive ion cores. An open-volume defect like a vacant lattice site is therefore an attractive center where the positron gets trapped. The reduced electron density at the vacant site increases the positron lifetime. In addition, the missing valence and core electrons cause substantial changes in the momentum distribution of the annihilated electrons. Two positron techniques have been efficiently used in defect studies in semiconductors, namely, the positron lifetime and the Doppler broadening of the $511 \mathrm{keV}$ line. There are three main advantages of positron annihilation spectroscopy which can be listed as follows. First, the identification of vacancytype defects is straightforward. Second, the technique is strongly supported by theory, since the annihilation characteristics can be calculated from first principles. Finally, positron annihilation can be applied to bulk crystals and thin layers of any electrical conduction type.

A special feature is that the positron can form a bound state with an electron in a system with low enough (local) electron density (Mohorovicic, 1934; Deutsch, 1951; Mogensen, 1995; Charlton and Humberston, 2001). This hydrogenlike quasiatom is called positronium (Ps), with a mass of $1.022 \mathrm{MeV} / c^{2}$ and a diameter of $1.06 \AA$ in its ground state in vacuum. The binding energy of Ps is $6.8 \mathrm{eV}$ in vacuum, i.e., half of the ionization energy of the hydrogen atom. Depending on the spin of the positron relative to the electron, Ps is in either the singlet (antiparallel spins, parapositronium, p-Ps) or triplet (parallel spins, orthopositronium, o-Ps) state. The self-annihilation properties of these two states are very different (Charlton and Humberston, 2001), and the so-called pick-off annihilation of o-Ps, in which the positron in Ps annihilates with an electron from the surroundings, prevails in matter (Brandt, Berko, and Walker, 1960). Importantly, the interaction between Ps and matter is predominantly repulsive due to the electron-electron repulsion. These properties are useful when porous media or soft condensed matter are studied with positron annihilation (Mogensen, 1995; Jean, Mallon, and Schrader, 2003).

\section{Positron annihilation methods}

Positrons can be created in several ways, of which the most common, in the case of laboratory-scale facilities, is using radioactive $\left(\beta^{+}\right)$isotopes, such as ${ }^{22} \mathrm{Na}$, which has relatively low intensity (up to $10^{9}$ positrons/s), but practical half-life of 2.6 years allowing reasonable use of the same source for 6-10 years. High-intensity sources (up to $10^{12}$ positrons/s) at large-scale facilities make use of pair production with the high-energy gamma flux created by a nuclear reactor (Hugenschmidt et al., 2004; Schut et al., 2004; Hawari et al., 2009) or a particle accelerator (Cassidy et al., 2009;
Krause-Rehberg et al., 2011). In both cases, the positrons have a wide and continuous energy spectrum with mean energies in the hundreds of $\mathrm{keV}$. These fast positrons can be used directly to probe the bulk (several hundreds of microns) of a material. In order to study thin films and coatings, the positrons need to be slowed down and if possible monochromated. Many crystal surfaces, such as those of heavy metal elements (e.g., W), have a negative work function for positrons, resulting in the fact that thermalized positrons within the solid can be emitted to the vacuum with an energy of a few eV if they reach the surface (Tong, 1972). These slow positrons can be magnetically guided and electrostatically accelerated to form a variable-energy beam allowing for, e.g., depth profiling.

The two most used methods in defect studies with positron annihilation are the positron lifetime spectroscopy and Doppler broadening (of the positron-electron annihilation radiation) spectroscopy. These techniques are very efficient in giving important information on vacancy defects in metals and semiconductors: the vacancy defects can be identified (sublattice in compounds, size in the case of vacancy clusters, and decoration by impurities), their charge states (in the case of semiconductors) can be determined, and their concentrations ${ }^{1}$ can be evaluated in the technologically important range from $10^{15}-10^{19} \mathrm{~cm}^{-3}$. Thanks to recent developments in theoretical calculations, computational studies can be directly compared with positron experiments, providing possibilities for detailed interpretations of experimental data. The positron lifetime and Doppler broadening techniques are also widely used in free-volume studies of molecular materials. Angular correlation of annihilation radiation (ACAR) (Beringer and Montgomery, 1942; Berko, Haghgooie, and Mader, 1977) is used to detect essentially the same phenomenon as Doppler broadening, namely, the momentum distribution of electronpositron annihilation radiation. The resolution of this technique is superior to Doppler broadening, but count rates are correspondingly lower. The better resolution of ACAR allows for detailed studies of the electronic structure (e.g., Fermi surfaces in metals), but does not bring significant improvements in the case of defect studies in semiconductors. It should be noted that these three techniques are easily used in bulk materials employing fast positrons and can be used with slow-positron beams, although requiring high intensity (except for Doppler broadening).

Positron-induced Auger electron spectroscopy (PAES) (Weiss et al., 1989; Soininen, Schwab, and Lynn, 1991) and reflection high-energy positron diffraction (RHEPD) (Ichimiya, 1992; Kawasuso and Okada, 1998) are extremely surface-sensitive positron-using techniques that require the use of a slow-positron beam, but do not require the measurement of positron-electron annihilation radiation. These techniques have important benefits compared to their "traditional" electron counterparts. In PAES the secondary-electron background is completely suppressed and the sensitivity is enhanced to only the first atomic layer (Weiss et al., 1989;

\footnotetext{
${ }^{1}$ Here a note on vocabulary is warranted: in the case of defects in semiconductors, it is typical to speak about concentrations when densities are meant. Hence concentrations are given in the units of $\mathrm{cm}^{-3}$ instead of ppm or ppb.
} 
Jensen and Weiss, 1990; Soininen, Schwab, and Lynn, 1991; Hugenschmidt, Mayer, and Schreckenbach, 2010). In RHEPD the positron crystal potential and the positive charge of the positron give rise to total reflection below a critical angle, again resulting in enhanced sensitivity to only the topmost atomic layer at the surface (Kawasuso and Okada, 1998; Kawasuso et al., 2003; Fukaya, Mochizuki, and Kawasuso, 2012). The drawback of these techniques is the necessity for a high-intensity positron beam as the measurement times with laboratory-scale low-intensity beams are far too long.

The main technological issues limiting further development of laboratory-scale experimental techniques are the poor efficiency of the moderation process when creating a slow-positron beam (resulting in the necessity for large-scale facilities with high-intensity sources) and the directional dispersion of the moderated positrons. The latter is partly responsible for the small number of scanning positron microprobes (SPMs), comparable to a scanning electron microscope (SEM), as the focusing of the beam with reasonable intensity even at a large-scale facility results in spot sizes of the order of $5 \mu \mathrm{m}$ (Greif et al., 1997; Triftshäuser et al., 1997). Another limitation for the SPM is the lateral straggling and the positron diffusion length of several hundreds of nanometers in a perfect crystal that will limit the spot size even if the focus is improved. The moderator efficiencies are in the $10^{-5}-10^{-4}$ range for passive crystalline heavy metal (e.g., W) moderators and in the $10^{-3}$ range for solid $\mathrm{Ne}$ moderators (Mills and Gullikson, 1986). As the latter need to be regenerated weekly, they are somewhat complicated to use. This holds, in particular, when the source and moderator are floated at a high voltage in order to have the sample grounded for easy manipulation. Sample manipulation (temperature, illumination, and bias control) is essential for sophisticated thin-film studies, and hence in many cases the $\mathrm{W}$ moderator is better suited.

Further technological limitations arise when the most powerful technique, positron lifetime spectroscopy, is used with slow-positron beams. The traditional technique depends on the existence of a start signal (given by the ${ }^{22} \mathrm{Na}$ source which emits practically simultaneously with the positron a high-energy 1.27 MeV photon). The moderation process strongly limits the usefulness of this start signal. Hence, either a positron-fly-by-detecting sensor must be installed or the beam must be tagged (e.g., by detecting secondary electrons ejected from the sample surface by positron impact) or modulated in time in order to retrieve a timing signal. The modulation of the positron beam has been shown to be the approach of choice, but requires radio-frequency beam bunching and chopping that have their own complications (Mills, 1980; Schödlbauer et al., 1987; Suzuki et al., 1992; Tashiro et al., 2001; Reurings and Laakso, 2007). The advantage is a time resolution good enough for studying semiconductors and metals where the positron lifetimes are an order of magnitude shorter than in molecular matter. In principle, the beam could be modulated also by trapping the positrons into a magnetic trap and releasing them at given time intervals. This approach is, however, better suited for applications where bunches containing a large amount of positrons are required, such as in experiments studying positron-positron interactions or molecular positronium (Cassidy and Mills, 2007). It should be noted that the defect-spectroscopic techniques based on the detection of positron-electron annihilation radiation rely on the nonexistence of positron-positron interactions. In these measurements, there is only one positron in the sample at any given time. As positrons in crystalline solids annihilate within a time frame of a few nanoseconds, a maximum intensity of about $10^{8}$ positrons/s is imposed.

\section{EXPERIMENTAL TECHNIQUES}

\section{A. Positrons in solids}

\section{Implantation, thermalization, and diffusion}

For a full description of the physics of positrons in solids, see Schultz and Lynn (1988) and Puska and Nieminen (1994). In the following we briefly describe the necessary concepts and models needed to analyze and interpret the experimental data.

The stopping profile of energetic positrons emitted by a radioactive $\left(\beta^{+}\right)$source is exponential (Brandt and Paulin, 1977):

$$
P(x)=\alpha \exp (-\alpha x), \quad \alpha \approx 16 \frac{\rho\left[\mathrm{g} / \mathrm{cm}^{3}\right]}{E_{\max }^{1.4}[\mathrm{MeV}]} \mathrm{cm}^{-1},
$$

where $\rho$ is the density of the solid and $E_{\max }$ is the maximum energy of the continuous $\beta^{+}$radiation spectrum. The most common isotope for positron experiments is ${ }^{22} \mathrm{Na}$ with $E_{\text {max }}=0.54 \mathrm{MeV}$. Hence for this isotope the characteristic penetration depth $1 / \alpha$ is, e.g., $110 \mu \mathrm{m}$ in $\mathrm{Si}$ and $40 \mu \mathrm{m}$ in $\mathrm{GaN}$. This means that positrons implanted directly as emitted from the source probe the bulk of a solid. It should be noted that the average energy of positrons emitted by ${ }^{22} \mathrm{Na}$ is $E_{\mathrm{av}}=$ $0.18 \mathrm{MeV}$. The (electronic) interactions during the stopping are the same for positrons as for electrons (Lennard et al., 1995), and hence in the case of semiconductors there is essentially no lattice damage caused by positron implantation. Additionally, a typical total fluence of at most $10^{12} \mathrm{~cm}^{-2}$ is implanted in the samples during an experiment. This is several orders of magnitude less than typically used in electron irradiation experiments with the purpose of creating lattice damage (Saarinen et al., 1995; Tuomisto, Ranki et al., 2007; Chen, Betsuyaku, and Kawasuso, 2008).

For monoenergetic positrons obtained from a low-energy positron beam (typical energies are below $50 \mathrm{keV}$ ), the stopping profile can be described by (Valkealahti and Nieminen, 1984)

$$
P(x)=-\frac{d}{d x} \exp \left[-\left(x / x_{0}\right)^{2}\right]
$$

where $x_{0}$ gives the peak position of the profile while the mean stopping depth is $\bar{x} \approx 0.886 x_{0}$ due to the asymmetry of the profile. The mean stopping depth is given as $\bar{x}=A E^{n}[\mathrm{keV}]$, where $A \approx 4 \times 10^{-6} / \rho\left[\mu \mathrm{g} / \mathrm{cm}^{2}\right]$ and $n \approx 1.6$. This description is very closely matched to the Monte Carlo simulations for stopping profiles of low-energy electrons (Shimizu and Ze-Jun, 1992; Ghosh and Aers, 1995; Dapor, 1996; Denison and Farrell, 2004; Nykänen et al., 2012). The mean stopping depth varies from a few nanometers up to a 
few micrometers. Hence low-energy positrons can be used to study near-surface layers and thin films.

The stopping and the ensuing thermalization of the positrons are fast processes, taking only $1-3$ ps at room temperature in both metals (Jensen and Walker, 1990) and semiconductors (Jorch, Lynn, and McMullen, 1984). This is considerably less than typical positron lifetimes of the order of $150-300$ ps. ACAR experiments have shown that the momentum distribution of annihilating positrons follows the sample temperature down to $10 \mathrm{~K}$ (Hyodo, McMullen, and Stewart, 1986). In a few cases incomplete thermalization may be important, e.g., positrons implanted at a very low energy can escape the sample nonthermally through the surface [see Gullikson and Mills (1986), Nielsen, Lynn, and Chen (1986), Huomo et al. (1987), and Lynn and Nielsen (1987) and there are also related problematics in measurements made for nanocrystalline matter; see Sec. V.C]. This needs to be taken into consideration when interpreting data from near-surface layers. After implantation and stopping the transport of thermalized positrons can be quite efficiently described by diffusion theory developed for free carriers (Bergersen et al., 1974). The positron diffusion coefficient in semiconductors at room temperature is typically $D_{+}=1-2 \mathrm{~cm}^{2} / \mathrm{V} \mathrm{s}$. The characteristic diffusion length during the positron lifetime $\tau$ is $L_{+}=\left(D_{+} \tau\right)^{1 / 2}=100-200 \mathrm{~nm}$.

\section{Positron states and trapping}

After implantation and thermalization the positron in a semiconductor is in a Bloch-like state in a perfect periodic crystal lattice. The thermalized positron at its ground state can be described to a good approximation (see Sec. III) by a single-particle Schrödinger equation

$$
-\frac{1}{2 m^{*}} \nabla^{2} \psi_{+}(\mathbf{r})+V(\mathbf{r}) \psi_{+}(\mathbf{r})=E_{+} \psi_{+}(\mathbf{r}),
$$

where the positron potential consists of an electrostatic Coulomb potential and a term that takes into account the electron-positron correlation effects. Because of the Coulomb repulsion from positive ion cores, the positron wave function is concentrated in the interstitial space between the atoms in the lattice. The positron energy band is parabolic and free particle like (Boev, Puska, and Nieminen, 1987). The effective mass of the positron is $m^{*} \approx 1.5 m_{0}$ due to phonons and the screening cloud of electrons (Bergersen and Pajanne, 1969).

The positron lifetime and the Doppler broadening of the annihilation radiation can also be calculated once the corresponding electronic structure of the solid system is known. The positron annihilation rate $\lambda$, the inverse of the positron lifetime $\tau$, can be thought to be proportional to the overlap of the electron and positron densities:

$$
\frac{1}{\tau}=\lambda=\pi r_{e}^{2} c \int\left|\psi_{+}(\mathbf{r})\right|^{2} n_{-}(\mathbf{r}) d \mathbf{r},
$$

where $r_{e}$ is the classical electron radius, $c$ is the velocity of light, and $n_{-}(\mathbf{r})$ is the electron density. The momentum distribution $\rho(\mathbf{p})$ of the annihilation radiation is a nonlocal quantity and requires knowledge of all electron wave functions $\psi_{i}$ overlapping with the positron. In the simplest approximation it can be written in the form

$$
\rho(\mathbf{p})=\pi r_{e}^{2} c \sum_{i}\left|\int d \mathbf{r} e^{-i \mathbf{p} \cdot \mathbf{r}} \psi_{+}(\mathbf{r}) \psi_{i}(\mathbf{r})\right|^{2},
$$

where the summation goes over occupied electron states. It should be noted that the momentum distribution $\rho(\mathbf{p})$ of the annihilation radiation is mainly that of the annihilating electrons "seen by the positron," because the momentum of the thermalized positron is negligible. The theoretical methods used to determine the positron's ground state and the annihilation parameters are reviewed in Sec. III.

In analogy to free carriers, the positron also has localized states at lattice imperfections. At vacancy-type defects where ions are missing, the repulsion sensed by the positron is lowered and the positron experiences these kinds of defects as potential wells. As a result, localized positron states at open-volume defects are formed. The positron ground state at a vacancy-type defect is generally deep; the binding energy is about $1 \mathrm{eV}$ or more (Makkonen and Puska, 2007). In a vacancy defect the electron density is locally reduced. This is reflected in the positron lifetime which is longer than in the defect-free lattice. Hence the positron lifetime measurement is a probe of vacancy defects in materials. Positron annihilation at a vacancy-type defect also leads to changes in the momentum distribution probed by the Doppler broadening experiment. The momentum distribution arising from valence electron annihilation becomes slightly narrower due to a lower electron density. In addition, the localized positron at a vacancy has a reduced overlap with ion cores leading to a considerable decrease in annihilation with high-momentum core electrons. The localized positron has time to interact with the host lattice during its lifetime of $>150 \mathrm{ps}$ and enlarge the average open volume of the vacancy by repelling neighboring positive ion cores.

A negatively charged impurity atom or an intrinsic point defect can bind positrons at shallow states even if these defects do not contain open volume. Being a positive particle, the positron can be localized at the hydrogenic (Rydberg) state of the Coulomb field around a negatively charged center. The situation is analogous to the binding of an electron to a shallow donor atom. The positron binding energy at the negative ion can be estimated from simple effective-mass theory:

$$
E_{\text {ion }}=13.6 \mathrm{eV} \frac{m^{*}}{m_{0} \epsilon} \frac{Z^{2}}{n^{2}}=10-100 \mathrm{meV},
$$

where $\epsilon$ is the dielectric constant, $m^{*}$ is the effective mass of the positron, $Z$ is the charge of the negative ion, and $n$ is the quantum number. With $Z=1-3$ and $n=1-4$, Eq. (6) typically yields $E_{\text {ion }}=10-100 \mathrm{meV}$, indicating that positrons can be thermally excited from the Rydberg states at 100-300 K.

The hydrogenic positron state around a negative ion has a typical extension of $10-100 \AA$ and thus positrons probe the same electron density as in the defect-free lattice. As a consequence, the annihilation characteristics (positron lifetime, momentum density of annihilating pairs) are not different from those in the lattice. Although the negative ions cannot be identified with the experimental parameters, information on their concentration can be obtained in the positron lifetime and Doppler broadening experiments when they compete with vacancies in positron trapping. 
The positron transition from a free Bloch state to a localized state at a defect is called positron trapping. The trapping is analogous to carrier capture. However, it must be fast enough to compete with annihilation. The positron trapping rate $\kappa_{D}$ into a defect $D$ is proportional to the defect concentration $[D], \kappa_{D}=\mu_{D}[D] / N_{\text {at }}$, where $N_{\text {at }}$ is the atomic density of the host lattice. The trapping coefficient $\mu_{D}$ depends on the defect and the host lattice. Since the positron binding energy at vacancies is typically $>1 \mathrm{eV}$, the thermal escape (detrapping) of positrons from the vacancies can usually be neglected. Because of the Coulomb repulsion, the trapping coefficient at positively charged vacancies is so small that the trapping does not occur during the short positron lifetime of a few hundred picoseconds (Puska, Corbel, and Nieminen, 1990). Therefore, the positron technique does not detect vacancies or other defects in their positive charge states. The trapping coefficient at neutral vacancies is typically $\mu_{D} \approx 10^{14}-10^{15} \mathrm{~s}^{-1}$, independent of temperature. The positron trapping coefficient at negative vacancies is typically $\mu_{D} \approx 10^{15}-10^{16} \mathrm{~s}^{-1}$ at $300 \mathrm{~K}$. The experimental fingerprint of a negative vacancy is the increase of $\mu_{D}$ with decreasing temperature. The $T^{-1 / 2}$ dependence of $\mu_{D}$ is simply due to the increase of the amplitude of the free-positron Coulomb wave in the presence of a negative defect as the thermal velocity of the positron decreases. The temperature dependence of $\mu_{D}$ allows one to experimentally distinguish negative vacancy defects from neutral ones.

The positron trapping coefficient $\mu_{\text {ion }}$ at the hydrogenic states around negative ions is of the same order of magnitude as that at negative vacancies. Furthermore, the trapping coefficient exhibits a similar $T^{-1 / 2}$ temperature dependence. Unlike in the case of vacancy defects, the thermal escape of positrons from the negative ions plays a crucial role at usual experimental temperatures. The principle of detailed balance yields the following expression for the ratio of detrapping and trapping rates (Manninen and Nieminen, 1981):

$$
\frac{\delta}{\kappa}=\frac{1}{c_{\mathrm{ion}}}\left(\frac{m^{*} k_{B} T}{2 \pi \hbar^{2}}\right)^{3 / 2} e^{-E_{\mathrm{ion}} / k_{B} T}
$$

where $c_{\text {ion }}$ is the concentration of the negative ions. Typically the negative ions (shallow traps) influence positron annihilation at low temperatures $(T<100 \mathrm{~K})$, but the ions are not observed at high temperatures $(T>300 \mathrm{~K})$, where the escape rate is high.

It is worth noting that the determination of absolute defect concentrations based on positron experiments depends directly on the knowledge of the trapping coefficient, while the comparison of defect concentrations (say, in two differently doped semiconductor samples) gives accurate proportions even when the trapping coefficient is not known. In extensively studied semiconductors, such as GaAs, GaN, and ZnO (Saarinen et al., 1995; Oila et al., 2003; Tuomisto, Saarinen, Look, and Farlow, 2005), the cross correlation of optical, electrical, and positron experiments has narrowed down the trapping coefficient of negatively charged cation vacancies to $\mu_{V-} \approx(2-3) \times 10^{15} \mathrm{~s}^{-1}$. Theory (Puska and Nieminen, 1994) predicts that the neutral-vacancy trapping coefficient should be a factor of 2-3 lower than that of negatively charged vacancies; hence often the value of $\mu_{V} \approx 1 \times 10^{15} \mathrm{~s}^{-1}$ is used for neutral vacancies.

\section{Trapping model}

The practical situation during a measurement, where only one positron at a time is in the sample, can be described by a relatively simple kinetic rate model (time-dependent diffusion equation):

$$
\frac{\partial n(\mathbf{r}, t)}{\partial t}=D \nabla^{2} n(\mathbf{r}, t)-\mu_{+} \nabla \cdot[n(\mathbf{r}, t) \mathbf{E}]-\lambda n(\mathbf{r}, t)+S,
$$

where $n(\mathbf{r}, t)$ is the probability density of finding a delocalized (free) positron at the position $\mathbf{r}$ and time $t, D$ is the diffusion coefficient, and $\mu_{+}$is the positron mobility. In the sink term $\lambda$ is the sum of the "free"-positron annihilation rate $\lambda_{B}$ and the trapping rates to the defects in the system $\lambda=$ $\lambda_{B}(\mathbf{r})+\sum_{i} \kappa_{i}(\mathbf{r})$. In principle $D$ and $\mu_{+}$can also be functions of the position $\mathbf{r}$, but only in layered systems where they are constant throughout the layer (similar to $\lambda_{B}$ ), while $\kappa_{i}(\mathbf{r})$ is smooth and follows the defect profile. In the simplest case the source term $S(\mathbf{r})$ vanishes, but if positrons are allowed to escape from the trapped states at the defects, the source term is non-negligible $\left(S=\sum_{i} \delta_{i} n_{D, i}\right)$. In practice the three spatial dimensions can be reduced to just one $(x)$, as both the lateral straggling and the spot size of the implanted positrons essentially result in the experimental data being spatially averaged in the plane perpendicular to the main implantation direction.

The initial condition $n(x, 0)$ is given by the positron implantation profile $P(x)$ (initially the positron is free, so the probability of finding a positron in a trapped state at a defect is initially $\equiv 0$ ), while the boundary conditions can be usually thought of as being those of a semi-infinite system assuming thick enough samples so that positrons implanted from one side do not reach the other:

$$
\begin{aligned}
& n(x, 0)=P(x), \\
& \lim _{x \rightarrow \infty} n(x, t)=0, \\
& \left.D \frac{\partial n}{\partial x}\right|_{x=0}-\mu_{+} E(0)=\nu n(0, t) .
\end{aligned}
$$

Here $\nu$ represents the positron transition rate to states at the sample surface. The simplest kind of experiment, where positrons emitted by a radioactive source are directly injected into a sample, further simplifies the above expressions. This is because the data are essentially averaged over a wide spread of implantation depths, effectively removing the spatial dimension from Eq. (8). The situation can then be described by a set of rate equations ( $N$ different defects):

$$
\begin{aligned}
& \frac{d n_{B}}{d t}=-\left(\lambda_{B}+\sum_{j} \kappa_{j}\right) n_{B}+\sum_{j} \delta_{j} n_{D, j}, \\
& \frac{d n_{D, j}}{d t}=\kappa_{j} n_{B}-\left(\lambda_{D, j}+\delta_{j}\right) n_{D, j} \quad(j=1, \ldots, N) .
\end{aligned}
$$

Here the probability of a positron being in the free state is $n_{B}(t)$ and the probability of it being in a trapped state at defect $j$ is $n_{D, j}(t)$. The corresponding annihilation, trapping, and escape rates are given by $\lambda_{B}, \lambda_{D, j}, \kappa_{j}$, and $\delta_{j}$, respectively. In practice $\delta_{j} \neq 0$ only for shallow positron traps (negative-iontype defects) at sufficiently high temperatures. The boundary 
and initial conditions in Eq. (9) are simplified to $n_{B}(0)=1$ and $n_{D, j}(0)=0$. As an example, in the case where positrons are trapped at one kind of vacancy defect $(V)$ and one kind of shallow trap $(s t)$, the above set of equations becomes

$$
\begin{aligned}
& \frac{d n_{B}}{d t}=-\left(\lambda_{B}+\kappa_{V}+\kappa_{s t}\right) n_{B}+\delta_{s t} n_{s t}, \\
& \frac{d n_{V}}{d t}=\kappa_{V} n_{B}-\lambda_{V} n_{V}, \\
& \frac{d n_{s t}}{d t}=\kappa_{s t} n_{B}-\left(\lambda_{s t}+\delta_{s t}\right) n_{s t} .
\end{aligned}
$$

Applying the initial condition, the above equations can be solved and the probability of a positron to be alive at time $t$ is obtained as

$$
n(t)=n_{B}(t)+n_{V}(t)+n_{s t}(t)=\sum_{i} I_{i} \exp \left(\lambda_{i} t\right) .
$$

This means that exponential decay should be observed in experiments. The experimental lifetime spectrum in fact measures the probability of positron annihilation in the time interval $t \cdots t+d t$, and hence the lifetime spectrum $-d n(t) / d t$ is in this case composed of a sum of three components. The fractions of positron annihilations at various states are in this example given by

$$
\begin{aligned}
& \eta_{B}=1-\eta_{V}-\eta_{s t}, \\
& \eta_{V}=\frac{\kappa_{V}}{\lambda_{B}+\kappa_{V}+\left(\kappa_{s t} / 1+\delta_{s t} / \lambda_{s t}\right)}, \\
& \eta_{s t}=\frac{\kappa_{s t}}{\left(1+\delta_{s t} / \lambda_{s t}\right)\left[\lambda_{B}+\kappa_{V}+\left(\kappa_{s t} / 1+\delta_{s t} / \lambda_{s t}\right)\right]} .
\end{aligned}
$$

These expressions are useful as they can be compared with experimentally determined time-averaged quantities such as the average positron lifetime and parameters describing the Doppler broadening of annihilation radiation, as these parameters $(P)$ measure the superposition of the annihilations over all positron states: $P=\eta_{B} P_{B}+$ $\sum_{j} \eta_{D, j} P_{D, j}$. Depth-resolved analysis of the latter is possible when using a conventional slow positron beam (Schultz and Lynn, 1988). Then one can employ the steady-state version of Eq. (8). Often, especially in the case of thin semiconductor epilayers where the vacancy defect concentrations tend to be high, the diffusion can be neglected altogether and the positron implantation profile in Eq. (2) provides a sufficient approximation of the depth distribution of the positron signal. However, in many cases solving the steadystate version of Eq. (8) and fitting it (van Veen et al., 1991) to data measured in layered structures provides additional insight to the experimental observations. For more detailed discussions about the trapping model, see, e.g., Saarinen, Hautojärvi, and Corbel (1998) and Krause-Rehberg and Leipner (1999).

\section{B. Positron lifetime spectroscopy}

\section{Experimental details}

A positron lifetime experiment can be performed in a relatively simple way by using a radioactive ${ }^{22} \mathrm{Na}$ positron source. ${ }^{22} \mathrm{Na}$ decays through the $\beta^{+}$process, producing a positron and a neutrino, leaving an excited ${ }^{22} \mathrm{Ne}$ nucleus that rapidly decays through the emission of a $1.2745 \mathrm{MeV}$ $\gamma$ photon. This photon can be used as a start signal for the positron lifetime measurement, while the stop signal is given by one of the two $511 \mathrm{keV}$ annihilation $\gamma$ photons.

In practice the positron source material is in the form of $\mathrm{NaCl}$ which is typically stored as a water solution. The experiment is performed by sandwiching the positron source between two identical sample pieces. This can be done either by depositing some of the $\mathrm{NaCl}$ directly on one of the samples, and then placing the other on top of it, or by first making a sealed positron source through packaging some of the $\mathrm{NaCl}$ in thin foil. Common foil choices are $\mathrm{Al}, \mathrm{Ni}$, and sometimes a polymer such as Kapton. The packaging solution is preferable for reuse of source material, while the metal foils allow for a wider range of measurement temperatures. The source package needs to be made as thin as possible in order to ensure that a maximal fraction of positrons emitted by the source enters the samples and that as few positrons as possible annihilate in the source itself: e.g., typical Al-foil thickness that is used is $1.5 \mu \mathrm{m}$, and at most two layers of foil are on each side of the deposited $\mathrm{NaCl}$. Typical activity of such a source is $10-30 \mu \mathrm{Ci}\left[(0.3-1) \times 10^{6} \mathrm{~Bq}\right]$. Such a sample-source-sample sandwich can then be placed on a sample holder connected to a temperature control system, and the experiment can be designed in such a way as to allow for, e.g., sample illumination.

The lifetime experiment itself is performed by detecting the $\gamma$ photons serving as start and stop signals with two relatively large scintillating detectors (lateral dimensions and thicknesses of the order of centimeters) coupled with photomultiplier tubes [see, e.g., Nissilä et al. (2001)], each of which is tuned and optimized for one of the two photons. Detector geometry is optimized with respect to overall efficiency (covering as wide a fraction of the solid angle as possible), taking into account restrictions imposed by different scintillator materials. As an example, plastic scintillators can be used in a simple collinear geometry where the sample-source-sample sandwich is placed on the axis defined by the two detectors and the detectors are put as close to each other as possible. On the other hand, special care needs to be taken when using $\mathrm{BaF}_{2}$ scintillators, which benefit from a significantly higher detection efficiency and enable better resolution, but whose high- $Z \mathrm{Ba}$ causes strong $\gamma$ scattering (Becvar et al., 2000).

In a conventional lifetime measurement the two detector signals are analyzed with analog nuclear instrumentation electronics: constant fraction discriminators to choose photons of correct energy, a time-to-amplitude converter, letting through only pulses spaced close enough in time, and a multichannel analyzer (MCA) connected to a measurement computer. The result is a histogram of annihilation events as a function of time differences between the start and stop signals, i.e., the positron lifetime spectrum. Typical time intervals for each MCA channel are of the order of $25 \mathrm{ps}$. The modern and considerably cheaper way of doing the same is through direct digitization (fast analog-to-digital conversion) of the detector pulses and performing the signal analysis by software (Rytsölä et al., 2002; Saito et al., 2002; Nissilä et al., 2005; Becvar, Cizek, and Prochazka, 2008). The advantage of 
the digitized measurement is that it also allows for novel functionalities such as postmeasurement signal analysis (Rytsölä et al., 2002; Becvar, Cizek, and Prochazka, 2008) and, e.g., efficient pump-probe measurements with high frequencies (Mäki, Tuomisto et al., 2011; Mäki et al., 2012).

Figure 2 shows a typical lifetime spectrum collected with a positron lifetime spectrometer, containing roughly $2 \times 10^{6}$ detected emission-annihilation (lifetime) events. The exponential functional form is clearly visible, but there are a few features that need to be understood prior to analyzing the data in detail. These are (i) the background noise, (ii) the time resolution, and (iii) annihilations in the source. At first glance the existence of background noise is striking, as this is a true coincidence measurement and there should not be any random background noise. However, positrons are not emitted by the source in a deterministic way, and even if the source activity is only $10^{6} \mathrm{~Bq}$, which gives an average time difference of $1 \mu$ s between two decays, some of the positrons are emitted very rapidly one after the other and produce false coincidences. Indeed, the background level in this kind of positron lifetime experiment is completely determined by the source activity (Knoll, 2000) and the peak-to-background ratio can be improved only by reducing the source activity (and increasing the measurement time).

It is evident from Fig. 2 that the transition from the lefthand-side background to the event peak around $t=0$ is far from being sharp, indicating that the time resolution has a non-negligible width on the scale of the measurement. This means that the experimental data need to be modeled by the sum of exponentials (17) convoluted with the timing resolution function of the measurement setup. Dominant effects on the timing resolution come from the size of the scintillators (this is partially an optimization problem) and the settings on the photomultiplier tubes (Becvar et al., 2000; Nissilä et al., 2001). The functional form of the timing resolution is important as well: a Gaussian form (over sufficiently many orders of magnitude) indicates that the setup produces only statistical error in the measurement, does not alter the exponential decay components, and in addition makes data analysis simpler as fewer parameters need to be fitted or

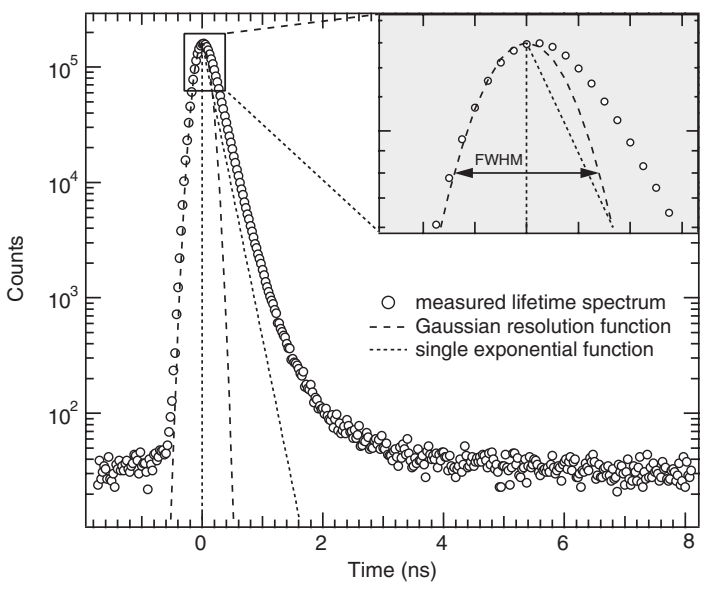

FIG. 2. Positron lifetime spectrum obtained with a typical spectrometer. The dashed and dotted lines show the detector resolution and the "ideal" spectrum. The inset shows a magnification of the $t=0$ range. determined. In Fig. 2 the resolution function of a typical setup is shown together with the lifetime spectrum. The full width at half maximum (FWHM) defining the Gaussian resolution function is $250 \mathrm{ps}$. It should be noted that the exponential decay component, seen as a slope on the semilogarithmic plot, corresponds to a lifetime of $160 \mathrm{ps}$. A Gaussian resolution function with FWHM of 250 ps allows for reliable determination of exponential decay components down to roughly $30 \mathrm{ps}$ as long as their intensity is high enough, while the narrowest (non-Gaussian) resolutions achieved in experimental setups go down to $\sim 140 \mathrm{ps}$ (Becvar, Cizek, and Prochazka, 2008). The inset in Fig. 2 points out another important aspect of the resolution function: the data close to $t=0$ are completely defined by the functional form of the resolution function, and hence very rapid decays with low relative intensity (when several decay components are present) cannot be reliably analyzed.

The annihilations in the positron source cause small distortions in the measured lifetime spectrum and need to be taken into account when analyzing the data. Figures 3 and 4 show three kinds of spectra: the "raw" data (Fig. 3), two spectra with subtracted background (upper panel of Fig. 4), and finally the same two spectra with subtracted source components (lower panel of Fig. 4). As an example, the exponential decay components produced by the source (and by the method of the measurement itself) in the case of an $\mathrm{Al}$-sealed ${ }^{22} \mathrm{Na}$ source can be approximated as one component coming from the heavily dislocated Al foil, the $\mathrm{NaCl}$, and surface effects at the interfaces between $\mathrm{NaCl}$ and $\mathrm{Al}$, and $\mathrm{Al}$ and the samples. The contribution of the $\mathrm{Al}$ foil can be estimated from the average $Z$ of the sample and the thickness of the foil (Bertolaccini and Zappa, 1967), as the backscattering of positrons from the sample surface strongly influences the probability of positron stopping in the Al foil. The corresponding lifetime component is $210-215 \mathrm{ps}$, and for typical semiconductors, the relative intensity is $1 \%-3 \%$. The $\mathrm{NaCl}$ and the surface effects produce two kinds of lifetime components: roughly 400 and 1500 ps. The latter is caused by positronium formation at the surfaces, and its importance can be estimated through comparison of background determined by fitting and averaging. For a carefully made source and

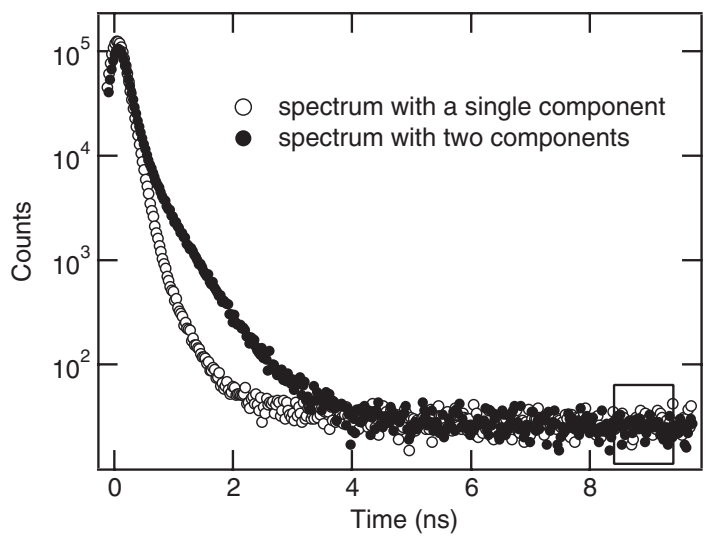

FIG. 3. Two "raw" positron lifetime spectra measured in different samples. The spectra are shown without the left-hand-side background, as in practice the window for optimal analysis starts around $t \approx-0.1 \mathrm{~ns}$. 


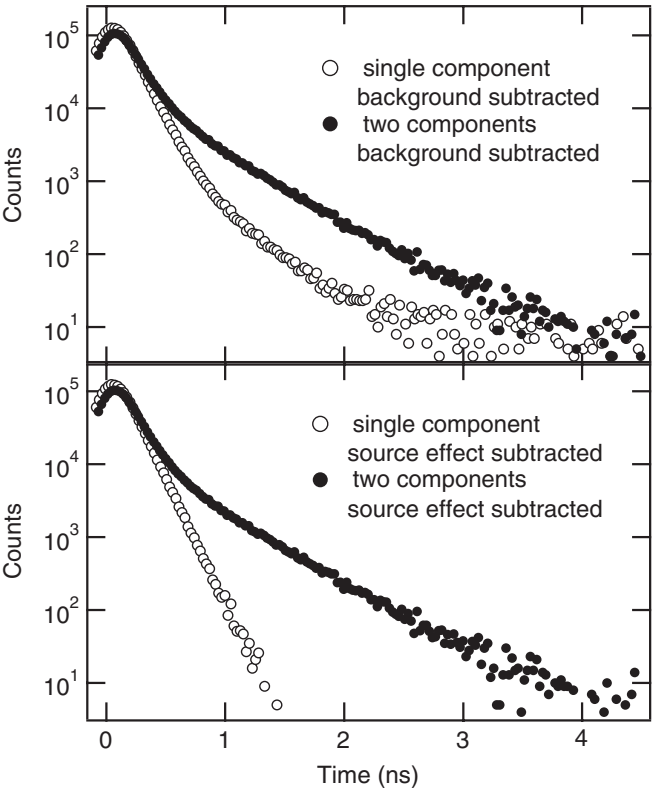

FIG. 4. Single- and two-component positron lifetime spectra after background subtraction (upper panel) and subsequent source effect subtraction (lower panel).

sample-source-sample sandwich the relative intensity of this component can be as low as $0.05 \%$, but is still meaningful due to the long lifetime. The 400 ps component is often the hardest to evaluate and requires high-quality reference samples where one can safely assume that no positron trapping at vacancy defects should be observed. On the other hand, the relative intensity of this component is usually in the range of $2 \%-6 \%$, making it rather easily fittable. The value depends strongly on the material and sample surface quality.

The source corrections, even if typically their total intensity in the measured spectrum is only a few percent, are at the root of the positron experiments being an inherently comparative technique, where changes of some sort are monitored. These changes can be induced, e.g., by change of the measurement temperature or by difference in the defect content (either identity or density) between samples of the same material. This comparative nature is further elucidated when the analysis of the lifetime data is considered.

\section{Data analysis}

Following Eq. (17), the experimental positron lifetime spectrum can be expressed as the convolution of the preferably Gaussian resolution function $R(t)$ with the sum of exponential functions:

$$
N(t)=\int_{-\infty}^{\infty} d s R(s)\left(-\frac{d n}{d t}(t-s)\right) .
$$

The constant background can simply be neglected in the following considerations, as its subtraction does not affect the convolution. The Gaussian form of the resolution function has the benefit of not affecting the time expectation value (center of mass) of the spectrum, and of not affecting the values of exponential components. In practice, Eq. (21) can be fitted to the experimental data by assuming a number of exponential components. Components are reliably fitted when the annihilation rates (or lifetimes) differ by at least a factor of 1.3-1.5, meaning that usually at most three components can be extracted. The fitting parameters include the FWHM of the resolution function and the intensities $I_{i}$ and annihilation rates $\lambda_{i}$ (slopes on the semilogarithmic plot) of the lifetime components. The subtraction of source components is performed through the procedure explained above, prior to final analysis of the data.

In addition to the relatively straightforward fitting of Eq. (21) to the experimental data, more sophisticated methods of data analysis can be employed. The two most prominent approaches are based on the inverse Laplace transform of Eq. (21) (van Resandt, Vogel, and Provencher, 1982; Gregory and Zhu, 1990) and on the Bayesian-probabilityinspired maximum-entropy method (Hoffmann et al., 1993; Shukla, Peter, and Hoffmann, 1993). These two methods have an important common feature, i.e., the number of lifetime components to be found is not a priori fixed. In addition, both methods are in principle better suited to cases where instead of discrete lifetime components, continuous lifetime distributions can be expected. The higher level of sophistication compared to the straightforward least-squares fitting makes these methods also somewhat more sensitive to noise in the experimental data.

Figure 3 shows two raw lifetime spectra (each with $2 \times 10^{6}$ annihilation events) measured in two exemplar samples. Evidently the spectrum plotted with full circles has an additional component compared to the one plotted with open circles. After $4 \mathrm{~ns}$, a background is reached in both spectra, and both the background and the intensity of the 1500 ps component can be determined from the data around $9 \mathrm{~ns}$ (denoted by the rectangle in the figure). Figure 4 shows the same lifetime spectra, the upper panel after background subtraction and the lower panel after subsequent source component subtraction (215 ps, $2.8 \%$; $400 \mathrm{ps}, 3.8 \%$; and $1500 \mathrm{ps}, 0.08 \%$ ). Clearly the data with open circles exhibit only one exponential component, while two exponential components can be seen in the data represented by full circles.

Fitting the single-component data gives $1 / \lambda_{1}=\tau_{1}=$ $110 \pm 1 \mathrm{ps}$, which coincides with the "center of mass" of the spectrum. This measurement is from a natural diamond sample (Mäki et al., 2009), and the result is typical of that material. Fitting the two-component data gives $1 / \lambda_{1}=\tau_{1}=$ $125 \pm 5 \mathrm{ps}, \quad 1 / \lambda_{2}=\tau_{2}=420 \pm 20 \mathrm{ps}, \quad$ and $\quad I_{2}=1-I_{1}=$ $(35 \pm 1) \%$, where the error bars are due to statistical uncertainty in the fitting. The two components mean that positrons annihilate at two distinct states: the simplest assumption is that one of them is the delocalized state in the lattice and the other a localized state at a (large) vacancy. ${ }^{2}$ Hence the set of Eqs. (14) is reduced to two equations:

$$
\frac{d n_{B}(t)}{d t}=-\lambda_{B} n_{B}-\kappa_{V} n_{B}
$$

\footnotetext{
${ }^{2}$ In fact, here this is not strictly the case-the 125 ps lifetime component is a mixture of the reduced bulk lifetime and the positron lifetime in dislocations (Mäki et al., 2009). We omit this detail for the sake of simplicity of the demonstration of how the reasoning proceeds.
} 


$$
\frac{d n_{V}(t)}{d t}=-\lambda_{V} n_{V}+\kappa_{V} n_{B}
$$

The full solution of this pair of equations [remembering that $n_{B}(0)=1$ and $n_{V}(0)=0$ ] is of the form

$$
n(t)=I_{1} \exp \left(-\lambda_{1} t\right)+I_{2} \exp \left(-\lambda_{2} t\right)
$$

where $\lambda_{1}=\lambda_{B}+\kappa_{V}$ and $\lambda_{2}=\lambda_{V}$. Hence the longer of the two experimentally determined components is directly the lifetime specific to the vacancy defects in question $\tau_{2}=\tau_{V}$, while the shorter of the components is the "reduced bulk lifetime," where the apparent positron lifetime in the lattice is shortened by the trapping process: $\tau_{1}=\lambda_{1}^{-1}=\left(\tau_{B}^{-1}+\kappa_{V}\right)^{-1}$.

The large number of annihilation events and the stability of the state-of-the-art positron lifetime spectrometers make the center of mass of the lifetime spectrum a highly accurate quantity: the standard deviation is as low as 0.2 ps (Tuomisto, Saarinen, Look, and Farlow, 2005). The importance of this parameter is accentuated by the fact that it coincides with the average positron lifetime defined by the annihilation fractions [Eq. (18)]:

$$
\tau_{\mathrm{av}}=\eta_{B} \tau_{B}+\eta_{V} \tau_{V}=\tau_{\text {c.m. }}=I_{1} \tau_{1}+I_{2} \tau_{2}
$$

where $\eta_{B}+\eta_{V}=I_{1}+I_{2}=1$. This equation relates the experimental spectrum directly to the kinetic trapping model and gives the possibility of using the statistically accurate average positron lifetime as a key parameter in lifetime analysis. Further, the statistical accuracy of $\tau_{\text {av }}$ provides the possibility of using it in data interpretation even in cases where the fitting of the experimental data represents only a best fit without physical interpretation. In the two-component data considered here we have $\tau_{\mathrm{av}}=232 \mathrm{ps}$.

In the simple case considered here the annihilation fractions are reduced to

$$
\eta_{B}=\frac{\lambda_{B}}{\lambda_{B}+\kappa_{V}} \quad \text { and } \quad \eta_{V}=\frac{\kappa_{V}}{\lambda_{B}+\kappa_{V}} .
$$

The experimentally determined parameters $\tau_{\mathrm{av}}, \tau_{V}$, and $\tau_{B}$ give now the possibility to estimate the trapping rate to the vacancy defect in question. The above considerations give

$$
\kappa_{V}=\lambda_{B} \frac{\tau_{\mathrm{av}}-\tau_{B}}{\tau_{V}-\tau_{\mathrm{av}}}
$$

Hence the concentration of the vacancies can be determined from the experimental data. The form of Eq. (27), however, shows that the concentration determination relies on the knowledge of $\tau_{B}$, i.e., on having at hand a reference sample where no positron trapping at vacancy-type defects is observed. The absolute value of $\tau_{B}$ is less important, as variations between spectrometers and source corrections can easily produce a $5 \mathrm{ps}$ offset, but being able to measure a baseline with the setup and source at hand is crucial for the highest achievable accuracy near the lower sensitivity limit. At more elevated vacancy concentrations this is not as important. The sample in the two-component example is a single crystal $\mathrm{ZnO}$ sample grown in a way that produces a high concentration of relatively large vacancy clusters with $\tau_{B}=170 \mathrm{ps}$.

The sensitivity limit is given by the ability to distinguish the average lifetime from $\tau_{B}$. The absolute lowest limit is given by the statistical accuracy of $0.2 \mathrm{ps}$ of $\tau_{\mathrm{av}}$, but is in most cases limited to roughly $1 \mathrm{ps}$ due to the inherent uncertainty

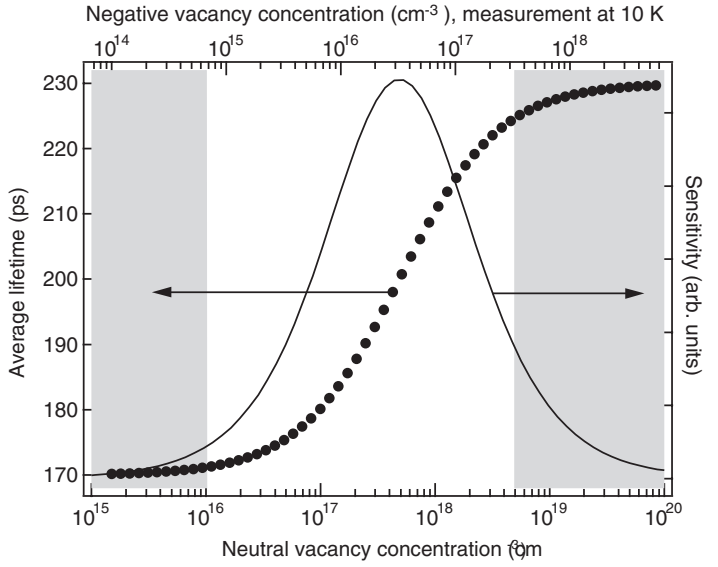

FIG. 5. Vacancy concentration sensitivity of the positron lifetime measurement in a system where $\tau_{B}=170$ and $\tau_{V}=230 \mathrm{ps}$. The highest sensitivity is midrange, where a small change in vacancy concentration produces a substantial change in the average positron lifetime.

in source corrections and reference reliability. The sensitivity is additionally a strong function of both $\tau_{\mathrm{av}}-\tau_{B}$ and $\tau_{V}-$ $\tau_{\text {av }}$ as seen in Fig. 5. It can be seen that the highest sensitivity to differences in vacancy concentrations is in the midrange of the changes in lifetimes, corresponding to roughly $10^{17} \mathrm{~cm}^{-3}$ in vacancy concentration. The lower limit for sensitivity is a result of reliably observing an increased average positron lifetime of 1 ps above $\tau_{B}$. The upper sensitivity limit is called saturation trapping, as it is due to all annihilations coming from trapped positrons and corresponds to a vacancy concentration of roughly $10^{19} \mathrm{~cm}^{-3}$. The limit is farther away from the absolute maximum of $\tau_{\mathrm{av}}=\tau_{V}$ due to the higher uncertainty in the fitting of the lifetime components: it is in practice impossible to distinguish the situation from saturation trapping when $\left|\tau_{\mathrm{av}}-\tau_{V}\right|<10 \mathrm{ps}$.

The above difficulties related to the source-originated background level and to the source corrections can be largely avoided by performing the positron lifetime experiment with a timestamped positron beam (Schödlbauer et al., 1987; Suzuki et al., 1992). The data analysis is, however, faced with other challenges such as an inherently non-Gaussian resolution function (even if very narrow) in pulsed beams (Reurings and Laakso, 2007). The time-stamped slow-positron beams have the benefit of enabling lifetime measurements in thin films, layered structures, and near-surface regions, but for a detailed analysis of the lifetime signals the full time-dependent positron diffusion equation [Eq. (8)] should be used. It should be noted that, due to their complexity, only a handful of positron beams capable of time-resolved experiments have been constructed.

\section{Information revealed by the positron lifetime}

The lifetime of a positron trapped in a vacancy is longer than in the "perfect" lattice. This is because the average electron density affecting the positron at the vacancy is lower than in the bulk, and correspondingly, the annihilation rate is lower. The lifetime increase for a monovacancy-sized defect is usually of the order of 20-40 ps (Saarinen et al., 1995, 1999) in elemental semiconductors and compound semiconductors where the sizes of the constituents are similar (e.g., GaAs). 
TABLE I. Positron lifetimes $(\tau)$ and relative core electron annihilation rates for the perfect bulk lattice and for the ideal vacancy clusters in $\mathrm{Si} . \lambda_{c}$, and $\lambda$ are the core and total annihilation rates, respectively. Reproduced from Hakala, Puska, and Nieminen (1998).

\begin{tabular}{lcc}
\hline \hline System & $\tau(\mathrm{ps})$ & $\lambda_{c} / \lambda(\%)$ \\
\hline Bulk & 221 & 2.19 \\
$V$ & 254 & 1.48 \\
$V_{2}$ & 299 & 0.97 \\
$V_{3}$ & 321 & 0.79 \\
$V_{4}$ & 330 & 0.72 \\
$V_{5}$ & 355 & 0.57 \\
\hline \hline
\end{tabular}

In size-mismatched compounds (e.g., GaN) the monovacancy of the larger atom can produce a lifetime that is 70-80 ps longer than in the bulk (Saarinen et al., 1997; Tuomisto et al., 2003), while quite typically the monovacancy of the smaller atom does not seem to trap positrons. As an example, Table I shows lifetimes calculated for small unrelaxed vacancy clusters in Si (Hakala, Puska, and Nieminen, 1998); for details on how calculations are performed, see Sec. III. The lifetime increases with increasing open volume of the vacancy. Simultaneously, the core annihilation fraction $\lambda_{c} / \lambda$, i.e., the fraction of annihilations with core electrons of all annihilation events, decreases, due to the reduced overlap between the positron density and the atomic core orbitals. The core annihilation fraction is typically quite low and thereby meaningless regarding the positron lifetime. However, the effect of the core electrons is more significant in the high-momentum region of the momentum density of annihilating electronpositron pairs (see Sec. II.C), where the chemical information is typically contained. For vacancy clusters larger than 4-5 missing atoms, the positron lifetime is already relatively insensitive to the size. In fact, as the size of the vacancy cluster increases, the lifetime saturates toward the lifetime of the negative positronium ion (or the spin-averaged positronium lifetime), 479 ps (Mills, 1981; Ceeh et al., 2011).

Positron lifetimes of singlet and triplet electron-positron states at paramagnetic vacancy defects have been considered theoretically (Alatalo, Puska, and Nieminen, 1993) but for Si and GaAs it was concluded that the splitting in the defect lifetime component is too small to be observed.

In general, with the exception of small vacancy defects such as monovacancies and divacancies, the positron lifetime can give only an order-of-magnitude estimate of a vacancy defect's size. Further, and most importantly, it is very insensitive to the chemical environments of the detected vacancy defects. For unambiguous identification of the defects, coincidence Doppler broadening measurements (see Sec. Sec. II.C) of the high-momentum part of the momentum density of annihilating positron-electron pairs are needed. Also one needs to be able to understand the indirect information contained in the measured data. Theoretical modeling is especially helpful in this regard.

\section{Doppler broadening spectroscopy}

\section{Experimental details}

Doppler broadening spectroscopy does not require timeresolved experiments and is hence applied regularly in both the sample-source-sample sandwich kind of experiments described in the previous section and with slow-positron beams. The experimental procedure when performing measurements with slow-positron beams is rather straightforward after such a beam has been constructed. The positron energy can be electrostatically tuned, typically in the range $0.1-50 \mathrm{keV}$. The annihilation photons are detected with high-purity Ge (HPGe) detectors that possess a high energy resolution. In practice the detector signals are collected to a computer with a MCA, while digitization of Doppler broadening experiments is a work in progress. One of the most important aspects in the instrumentation is effective stabilization of the peak position through online tuning of amplifier gain.

The motion of the annihilating electron-positron pair causes a Doppler shift in the annihilation radiation $\Delta E=$ $c p_{L} / 2$, where $p_{L}$ is the longitudinal momentum component of the pair in the direction of the $511 \mathrm{keV}$ annihilation photon emission:

$$
\rho\left(p_{L}\right)=\iint d p_{x} d p_{y} \rho(\mathbf{p}) .
$$

The electron momentum distribution $\rho(\mathbf{p})$ causes the broadening of the $511 \mathrm{keV}$ annihilation line. The shape of the $511 \mathrm{keV}$ peak gives thus the one-dimensional momentum distribution $\rho\left(p_{L}\right)$ of the annihilating electron-positron pairs. A Doppler shift of $1 \mathrm{keV}$ corresponds to a momentum value of 0.54 atomic units (a.u.). Another unit used traditionally is $1 \mathrm{mrad}\left(=10^{-3} m_{0} c\right)$, with the correspondence $1 \mathrm{keV}=$ $3.91 \mathrm{mrad}$. This geometric unit has its origin in ACAR experiments that can be used to detect the same effects, i.e., the electron momentum distribution. The momentum resolution in these experiments is better than in the energy-resolved experiments, but the detection efficiency is much lower. As in defect spectroscopy it is often necessary to perform large series of measurements, e.g., as a function of temperature or detection depth, and Doppler broadening spectroscopy has been the method of choice. ACAR and 2D-ACAR have been used in defect studies much less (Saito, Oshiyama, and Tanigawa, 1991; Ambigapathy et al., 1994).

The typical resolution of a HPGe detector is around $1-1.5 \mathrm{keV}$ at $511 \mathrm{keV}$. This is considerable compared to the total width of 2-3 keV of the annihilation peak, meaning that the experimental line shape is strongly influenced by the detector resolution (see Fig. 6). In addition, the peak-to-background ratio is rather low, only about $10^{2}$. Therefore, various shape parameters, where parts of the annihilation peak are integrated, are used to characterize the $511 \mathrm{keV}$ line. These parameters are explained in more detail in Sec. II.C.2.

The resolution and peak-to-background ratio of the Doppler broadening measurement can be significantly improved by measuring the annihilation radiation with two (collinear) detectors and imposing coincidence conditions (Alatalo et al., 1995; Asoka-Kumar et al., 1996; Szpala et al., 1996). The collinearity of the detectors imposes the first condition: the two annihilation photons need to be emitted in roughly opposite directions. The second condition is the time coincidence: the two annihilation photons need to originate from the same annihilation event. These two conditions alone improve the peak-to-background ratio to about $10^{4}$. The finest improvement comes, however, from the third condition and is obtainable only when two HPGe detectors 


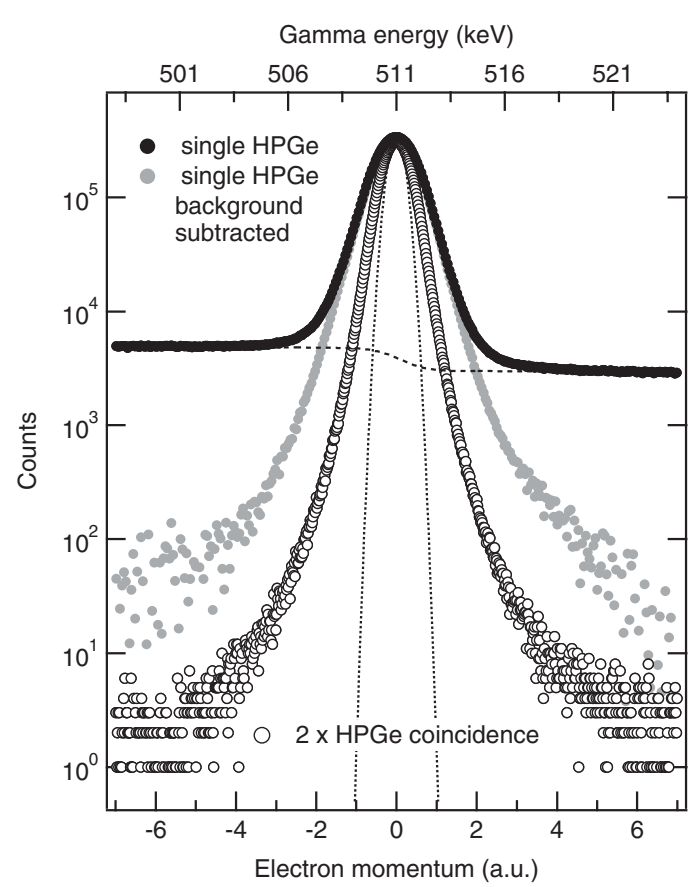

FIG. 6. Doppler broadening spectra obtained with a single HPGe detector and with two HPGe detectors in coincidence. The high background in the single-HPGe-detector measurement can be subtracted; the functional form is dictated by the incomplete charge collection in the detector (dashed line). The resolution function of a typical HPGe detector is also shown for reference (dotted line).

are used (the first two conditions can be achieved by using one HPGe detector and another, say, scintillating detector with poorer energy resolution to gate the HPGe detector). This requires that the sum of the energies $E_{1}$ and $E_{2}$ of the two coincidentally observed annihilation photons obeys

$$
E_{1}+E_{2}=2 m_{0} c^{2}-E_{+,-} \approx 1.022 \mathrm{MeV},
$$

i.e., the photons carry the rest mass of the positron and electron (each $511 \mathrm{keV}$ ) reduced by the binding energy of the pair $E_{+,-}$, which can in fact be neglected as it is of the order of a few eV. This kind of two-detector measurements produces a two-dimensional matrix, where on one diagonal one finds the coincidence spectrum with an improved peakto-background ratio of about $10^{6}$, while the real resolution function of the setup can be found on the other diagonal. The resolution of the measurement is in fact narrowed by a factor of $\sim \sqrt{2}$ compared to the resolution of the individual detectors. More details about the procedures can be found in, e.g., Asoka-Kumar et al. (1996). Figure 6 shows the Dopplerbroadened annihilation peak measured with one HPGe detector, and the same sample measured with a two-HPGe detector coincidence system (data integrated from the diagonal of the 2D matrix). The high background in the single-HPGe-detector data can be subtracted to some extent (dashed line in Fig. 6) prior to analysis.

\section{Data analysis}

The low-momentum shape parameter $S$ is defined as the ratio of the counts in the central region of the annihilation line (see the upper panel of Fig. 7) to the total number

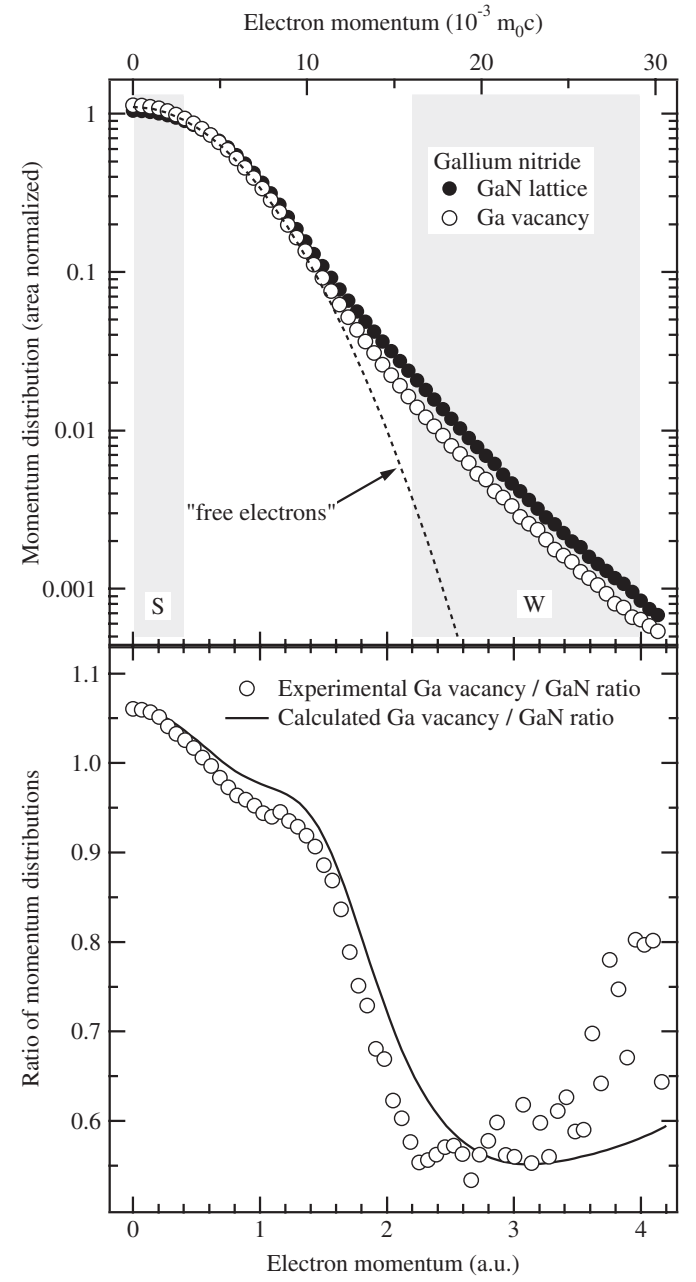

FIG. 7. Folded experimental coincidence Doppler broadening spectra for the GaN lattice and $\mathrm{Ga}$ vacancy. Typical integration windows for the $S$ and $W$ parameters are shown (upper panel). Experimental and calculated "ratio curves" of the momentum densities (lower panel).

of the counts in the line. In the same way, the highmomentum parameter $W$ is the fraction of the counts in the wing regions of the line. Because of their low momenta (low degree of localization), mainly valence electrons contribute to the region of the $S$ parameter. On the other hand, only core electrons have momentum values high enough to contribute to the $W$ parameter. Therefore, $S$ and $W$ are sometimes called the valence and core annihilation parameters, respectively. The practical choice of the integration windows for the $S$ and $W$ parameters is straightforward. The $S$ parameter is defined in such a way that it retains the statistical accuracy of collecting a large number of annihilation events (typically roughly $10^{6}$ ) to the peak: usually $S \approx 0.5$. The lower limit of the $W$ parameter integration window is chosen far enough from the peak center in order to have a minimal contribution from the free-electron distribution shown in Fig. 7. Prior to parameter integration, the background is subtracted (shown with a dashed curve in Fig. 6). The functional form is due to incomplete charge collection in the HPGe detectors, resulting in the background level at a given energy being proportional to the number of events at higher energies (Knoll, 2000). 
The $S$ and $W$ parameters are time-averaged (or timeintegrated) quantities and thus behave similarly to the average positron lifetime. If saturation trapping at a certain kind of vacancy defect produces parameters $S_{V}, W_{V}$ and a reference sample produces parameters $S_{B}, W_{B}$, the measurement in a sample for which $\tau_{\text {ave }}$ is given by Eq. (25) will give

$$
S=\eta_{B} S_{B}+\eta_{V} S_{V}, \quad W=\eta_{B} W_{B}+\eta_{V} W_{V}
$$

As a consequence, if $S_{V}$ and $S_{B}$ are known, the vacancy concentration can also be determined from the $(S, W)$ parameters. Furthermore, the links between these parameters enable the combination of positron lifetime and Doppler broadening results and various correlations between $\tau_{\mathrm{av}}, S$, and $W$ can be studied. As an example, if a variety of samples with varying concentrations of the same vacancy defect is studied, the data will form a line in the $(S, W),\left(S, \tau_{\text {av }}\right)$, and $\left(W, \tau_{\text {av }}\right)$ planes. It should be noted that the Doppler experiment does not allow the direct extraction of defect-specific parameters in the same way as the positron lifetime experiment, as the data are always a superposition of annihilations in various states. However, the annihilation fractions that can be determined in positron lifetime experiments can be used to extract this information from Doppler data in some cases.

It is important to stress that the absolute values of the $S$ and $W$ parameters are meaningless. They depend strongly on the detector geometry, resolution, calibration, amplifier gain, and MCA channel width, and in some cases on the direction of measurement. The last point is in fact important: the crystal lattice contains natural anisotropy transferred to the electron momentum distribution, and measurements along different lattice directions give slightly different results. Hence it is even more important to perform comparative measurements and preferably possess a reference sample where no positron annihilation at vacancy defects is detected. In fact, it is quite typical to express the $S$ and $W$ parameters as normalized to the $S_{B}$ and $W_{B}$ obtained in a reference sample. This reduces the dependence of the data on the various aspects listed above to some extent, but not completely. As an example, even the normalized parameters depend strongly on the detector resolution. This means that data measured with one setup cannot be directly compared to those obtained with another setup; instead full analysis and interpretation of the results is necessary prior to comparison.

The coincidence Doppler data shown in Fig. 6 are typically folded along the 0-momentum line (the symmetry of the peak is evident), and adjacent points are summed together to improve statistics and reduce noise especially in the wing regions. The result is shown in the upper panel of Fig. 7. The high-momentum part of the Doppler broadening spectrum arises mainly from annihilations with core electrons and hence contains information on the chemical identity of the atoms close to the annihilation site of the positron. The difference between the data obtained in the GaN lattice and a Ga vacancy shown in Fig. 7 is evident, but otherwise the data look rather featureless. A similar normalization procedure as in the case of the $S$ and $W$ parameters, where the whole distribution is normalized to that of the reference (GaN lattice in this case), results in the so-called "ratio curve" shown in the lower panel of Fig. 7. This procedure reveals details of the distribution, such as the above-unity value of the Ga vacancy data at 0 momentum, the shoulder at $\sim 1.5$ a.u., and the low intensity above 2 a.u. The solid curve is calculated with methods explained in detail in Sec. III.

\section{Chemical information contained in Doppler spectra}

Coincidence Doppler broadening measurements made with a two-detector setup measure the momentum density of annihilating electron-positron pairs accurately up to a very high momentum of several atomic units or tens of $10^{-3} m_{0} c$. This region is dominated by annihilation with core electron shells. The specificity of the technique to different chemical elements is seen clearly in bulk measurements made for elemental metals and semiconductors (Asoka-Kumar et al., 1996; Myler and Simpson, 1997; Ghosh et al., 2000). The high-momentum region of the spectra contains information on the chemical elements close to the annihilation sites. In measurements probing vacancy defects, atoms in their surroundings can be distinguished from the host lattice atoms thanks to their differing core shells.

As a detailed example of chemical information contained in spectra measured for vacancy-dopant complexes in semiconductors, and to show how first-principles modeling can help to understand experimental results, we consider a work on identification of vacancy-antimony complexes in highly Sb-doped Si (Rummukainen et al., 2005). In this study, atomic relaxations of defects were neglected for the most part. The inward relaxation of the vacancy defects predicted by the LDA was assumed to be canceled by the repulsive force of the positron on the neighboring ions. Figure 8 shows

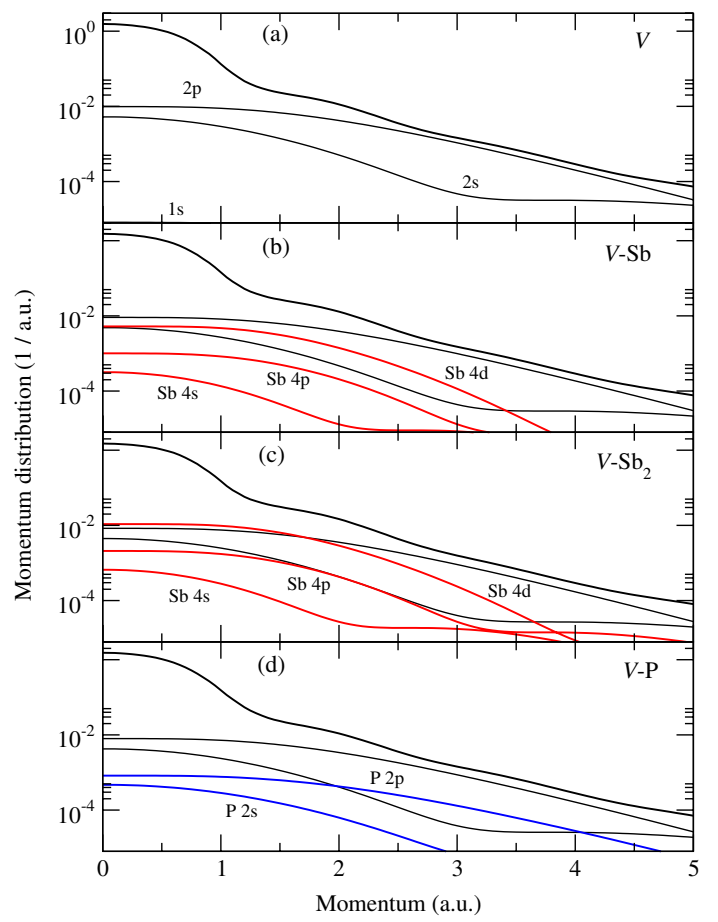

FIG. 8 (color online). Computational Doppler spectra of (a) $V$, (b) $V$ - Sb, (c) $V-\mathrm{Sb}_{2}$, and (d) $V$-P in Si. The thick (black) solid line shows the full spectrum. The contributions of individual $\mathrm{Si}$ core shells are shown with thin (black) solid lines, and dopant atoms' core shell contributions by solid colored lines. Adapted from Rummukainen et al., 2005. 
Doppler spectra calculated for the monovacancy $(V)$ in $\mathrm{Si}$ as well as for the vacancy-antimony and vacancy-phosphorus complexes $V$-Sb, $V$-Sb $\mathrm{Sb}_{2}$, and $V$-P. The neglect of atomic relaxations enables us to focus solely on the chemical information contained in the spectra. In this case and in most other systems containing atoms of more than one kind, the effect of the chemical surroundings and differing core electron orbitals is stronger than that of the detailed atomic geometry of the defects.

Figures 8(a)-8(d) show for $V, V$-Sb, $V$ - $\mathrm{Sb}_{2}$, and $V$-P, respectively, the total spectrum (thick solid lines) as well as the contributions of the dominant core shells. At high momenta, the dominating shell is the $\mathrm{Si} 2 p$ in all systems. For the vacancy-dopant complexes the contributions of the impurity atoms' core shells are also shown (thick colored lines). This calculation, like many others, is made using the model of Alatalo et al. (1996) [see Eqs. (51) and (52)] and using a parametrized positron wave function for annihilating core electrons. Hence, the contributions of core shells look the same on the logarithmic scale irrespective of the system. Their magnitudes are determined by the respective partial annihilation rates $\lambda_{j}^{\mathrm{LDA}}$. Since the core electron shells of $\mathrm{Si}$ and $\mathrm{P}$, neighboring elements on the periodic table, are from the positron's point of view rather similar, the spectra of $V$ and $V$-P nearly coincide. The case of $\mathrm{Sb}$ is quite different thanks to the presence of its $4 d$ shell. Its intensity in the Doppler spectrum increases with an increasing number of Sb nearest neighbors comparable to or even higher than that of the $\mathrm{Si} 2 p$ at and below 2 a.u. In a ratio spectrum [see Rummukainen et al. (2005)] the "Sb fingerprint" is seen as a peak at this momentum. The intensity of the peak correlates with the average number of $\mathrm{Sb}$ nearest neighbors around the monovacancy-sized vacancy complexes in the sample.

The momentum density of annihilating electron-positron pairs as measured by Doppler broadening or angular correlation measurements is, in general, more anisotropic for the positron's delocalized bulk state than for positrons trapped at vacancies. For covalently bonded semiconductors such as GaAs and Si (Saito, Oshiyama, and Tanigawa, 1991; Ambigapathy et al., 1994; Peng et al., 1994; Hakala, Puska, and Nieminen, 1998), the anisotropic part is significant. As already discussed, upon positron trapping the (normalized) spectrum becomes narrower due to increased relative contact with low-momentum valence electrons at the vacancy. Further, a vacancy's spectrum is more isotropic. On the one hand, this is because a localized positron does not sense much the ordered periodic lattice with its directed bonds. On the other hand, it is because possible asymmetric vacancy defects and defect clusters, which as such would produce an asymmetric momentum spectrum, are typically oriented randomly in the sample. The measurement averages over different orientations and the anisotropic spectra corresponding to individual orientations sum up to a more isotropic spectrum. When comparing experimental spectra with computational ones the averaging over orientations has to be performed in the modeling as well (Hakala, Puska, and Nieminen, 1998). The 2D-ACAR technique and uniaxial stress provided an additional means to observe the aligning of divacancies in $\mathrm{Si}$ in an experiment (Tang et al., 1997) paralleling the electron paramagnetic resonance study of Watkins and Corbett (1965).

\section{THEORY AND COMPUTATIONAL METHODS}

The first models of positrons in solids interacting with electrons were the electron gas models by Ferrell (1956), Kahana (1960, 1963), and Carbotte and Kahana (1965). The study proceeded to real inhomogeneous metals involving high-momentum (umklapp) components and core annihilation (Carbotte, 1966; Salvadori and Carbotte, 1969; Fujiwara, Hyodo, and Ohyama, 1972; Hede and Carbotte, 1972). Since the early 1970s and the first model for positron trapping (Hodges, 1970) defect studies with their trapped positrons became a separate line of research also on the theory side [for early work, see Arponen et al., 1973; Manninen et al., 1975; Gupta and Siegel, 1977; Hautojärvi et al., 1977; Gupta and Siegel, 1980a, 1980b]. In the theory and modeling aspect of this review, we focus mostly on theory and methods useful in theory-assisted defect identification, especially how to accurately predict positron lifetimes and Doppler spectra for direct comparisons with experiments. Concerning theory and models on what happens prior to the annihilation event (e.g., positron thermalization and trapping mechanisms and rates), see Puska and Nieminen (1994).

Important developments for the practical calculation of positron states and annihilation in solids from the defect studies point of view included in the 1970s and 1980s, for instance, the positron pseudopotential theory (Kubica and Stott, 1974; Nieminen, 1975; Stott and Kubica, 1975; Stott and West, 1978), two-component electron-positron density-functional models (Chakraborty, 1981; Chakraborty and Siegel, 1983; Nieminen, Boroński, and Lantto, 1985; Boroński and Nieminen, 1986), the simple but powerful atomic superposition method (Puska and Nieminen, 1983), and the applications of these to timely problems.

Section III.A discusses the formalism of the two-component electron-positron density-functional theory. Sections III.B and III.C go deeper into the practical modeling of trapped positrons and the measurable annihilation parameters such as positron lifetime and Doppler broadening. Section III.D discusses practical approximations used in the electron-positron densityfunctional calculations. Sections III.E and III.F discuss different levels of approximations concerning self-consistency and numerical implementation, most importantly the atomic superposition method and the application of different band-structure schemes to the positron problem.

\section{A. Two-component electron-positron density-functional theory}

The density-functional theory (DFT) (Hohenberg and Kohn, 1964) within the Kohn-Sham method (Kohn and Sham, 1965) is the main workhorse in first-principles materials modeling [see, e.g., Martin (2004) and Drabold and Estreicher (2007)]. Using supercell calculations one can predict bulk properties and properties of defects in solids, including their structure, formation enthalpies, diffusion barriers, ionization levels, etc. Most of the practical modeling of positrons annihilating at lattice defects has during the past decades been made using two-component electron-positron formulations of DFT (Chakraborty, 1981; Chakraborty and Siegel, 1983; Nieminen, Boroński, and Lantto, 1985; Boroński and Nieminen, 1986). Next we discuss the formalism as given by 
Boroński and Nieminen (1986). The two-component approach enables modeling of both delocalized and localized positrons, including the effect of the localized positron on the defect's surrounding electronic and ionic structure. Furthermore, the direct connection with the usual one-component DFT and its approximations, such as those for the exchange-correlation energy, makes it rather straightforward to implement electronpositron calculations in the codes used by the electronicstructure and materials-modeling communities. The use of state-of-the-art electronic-structure packages enables one also to easily gain, in addition to positron annihilation parameters such as positron lifetime or momentum density of annihilating electron-positron pairs, complementary information on the defects modeled.

In this section, we use the Hartree atomic units $(m=\hbar=$ $\left.e=4 \pi \epsilon_{0}=1\right)$.

In the two-component DFT for electron-positron systems, the fundamental quantities are the electron and positron densities $n_{-}(\mathbf{r})$ and $n_{+}(\mathbf{r})$. Practical calculations are based on a modified Kohn-Sham scheme, in which one models a noninteracting system with the electron and positron densities equal to those of the true interacting system using an energy functional ansatz of the form

$$
\begin{aligned}
E_{\mathrm{tot}}\left[n_{-}, n_{+}\right]= & F\left[n_{-}\right]+F\left[n_{+}\right]-\int d \mathbf{r} d \mathbf{r}^{\prime} \frac{n_{-}(\mathbf{r}) n_{+}\left(\mathbf{r}^{\prime}\right)}{\left|\mathbf{r}-\mathbf{r}^{\prime}\right|} \\
& +\int d \mathbf{r} V_{\mathrm{ext}}(\mathbf{r})\left[n_{-}(\mathbf{r})-n_{+}(\mathbf{r})\right] \\
& +E_{c}^{e^{-} p}\left[n_{-}, n_{+}\right]
\end{aligned}
$$

In Eq. (31) the third term is the classical electrostatic interaction between electrons and positrons. The fourth term is the interaction energy with the external potential (the nuclei are treated within the Born-Oppenheimer approximation). $F[n]$ is the one-component functional

$$
F[n]=T_{s}[n]+\frac{1}{2} \int d \mathbf{r} d \mathbf{r}^{\prime} \frac{n(\mathbf{r}) n\left(\mathbf{r}^{\prime}\right)}{\left|\mathbf{r}-\mathbf{r}^{\prime}\right|}+E_{\mathrm{xc}}[n],
$$

where $T_{s}[n]$ is the kinetic energy of noninteracting particles with density $n$ in the two-component system with densities $n_{-}(\mathbf{r})$ and $n_{+}(\mathbf{r})$, the second term is the Hartree interaction energy, and $E_{\mathrm{xc}}[n]$ is the exchange and correlation energy between particles of the same kind. Many-body electronpositron interactions are incorporated in the electron-positron correlation energy term $E_{c}^{e^{-} p}\left[n_{-}, n_{+}\right]$in Eq. (31). Minimization of the functional equation (31) with fixed numbers of electrons and positrons leads to the below singleparticle equations for electrons and positrons, respectively:

$$
\begin{aligned}
& -\frac{1}{2} \nabla^{2} \psi_{i}(\mathbf{r})+\left[\phi(\mathbf{r})+\frac{\delta E_{\mathrm{xc}}\left[n_{-}\right]}{\delta n_{-}(\mathbf{r})}+\frac{\delta E_{c}^{e-p}\left[n_{+}, n_{-}\right]}{\delta n_{-}(\mathbf{r})}\right] \psi_{i}(\mathbf{r}) \\
& \quad=\varepsilon_{i} \psi_{i}(\mathbf{r}), \\
& -\frac{1}{2} \nabla^{2} \psi_{i}^{+}(\mathbf{r})+\left[-\phi(\mathbf{r})+\frac{\delta E_{\mathrm{xc}}\left[n_{+}\right]}{\delta n_{+}(\mathbf{r})}+\frac{\delta E_{c}^{e-p}\left[n_{+}, n_{-}\right]}{\delta n_{+}(\mathbf{r})}\right] \\
& \quad \times \psi_{i}^{+}(\mathbf{r})=\varepsilon_{i}^{+} \psi_{i}^{+}(\mathbf{r}) .
\end{aligned}
$$

In Eqs. (33) and (34), the Hartree and external potentials are incorporated in the term

$$
\phi(\mathbf{r})=\int d \mathbf{r}^{\prime} \frac{n_{-}\left(\mathbf{r}^{\prime}\right)-n_{+}\left(\mathbf{r}^{\prime}\right)}{\left|\mathbf{r}-\mathbf{r}^{\prime}\right|}+V_{\text {ext }}(\mathbf{r}) .
$$

The densities are obtained by summing over the occupied orbitals as

$$
n_{-}(\mathbf{r})=\sum_{\mathrm{occ}}\left|\psi_{i}(\mathbf{r})\right|^{2}, \quad n_{+}(\mathbf{r})=\sum_{\mathrm{occ}}\left|\psi_{i}^{+}(\mathbf{r})\right|^{2} .
$$

Most often one is interested only in the typical experimental situation of having only one positron in the sample at any given time. Then the exchange-correlation energy associated with the positron corrects only for its Hartree self-interaction (Boroński and Nieminen, 1986),

$$
E_{\mathrm{xc}}\left[n_{+}\right]=-\frac{1}{2} \int d \mathbf{r} d \mathbf{r}^{\prime} \frac{n_{+}(\mathbf{r}) n_{+}\left(\mathbf{r}^{\prime}\right)}{\left|\mathbf{r}-\mathbf{r}^{\prime}\right|}
$$

The corresponding terms in the positron's potential cancel accordingly,

$$
\frac{\delta E_{\mathrm{xc}}\left[n_{+}\right]}{\delta n_{+}(\mathbf{r})}=-\int d \mathbf{r}^{\prime} \frac{n_{+}\left(\mathbf{r}^{\prime}\right)}{\left|\mathbf{r}-\mathbf{r}^{\prime}\right|} .
$$

In the case of a single delocalized positron in an infinite crystal, it is first assumed that the positron does not affect the average electron density $n_{-}(\mathbf{r})$. Second, the appropriate zero-positron-density limit of the electron-positron correlation energy is used (Boroński and Nieminen, 1986). After these assumptions, the two-component calculation can be done as follows:

(1) The electron density is determined first without the effect of the positron in a usual one-component DFT calculation.

(2) The positron's single-particle wave function is then solved in the potential

$$
V_{+}(\mathbf{r})=-\int d \mathbf{r}^{\prime} \frac{n_{-}\left(\mathbf{r}^{\prime}\right)}{\left|\mathbf{r}-\mathbf{r}^{\prime}\right|}-V_{\mathrm{ext}}(\mathbf{r})+V_{\mathrm{corr}}(\mathbf{r})
$$

where $V_{\text {corr }}(\mathbf{r})$ is the zero-positron-density limit of the electron-positron correlation potential $\delta E_{c}^{e^{-p}} / \delta n_{+}(\mathbf{r})$.

Once the electron and positron densities and orbitals are obtained, one can continue modeling the measurable positron annihilation characteristics such as the positron lifetime and the momentum density of annihilating pairs. This will be discussed in Sec. III.C.

\section{B. Modeling localized positrons}

When a positron is localized in a crystal defect, Eqs. (34)-(36) would in principle have to be solved selfconsistently. However, only a very limited number of fully self-consistent calculations have been made for localized positrons in atomistic systems (Gilgien et al., 1994; Puska, Seitsonen, and Nieminen, 1995; Saito and Oshiyama, 1996; Tang et al., 1997; Makhov and Lewis, 2005; Wiktor et al., 2013). Gilgien et al. used a modification of the original Boroński and Nieminen (1986) formulation, in which zerocomponent limits of the interaction functionals were taken even for a localized positron. They justified the procedure by explicit exclusion of self-interaction terms. This is how they attributed all positron potential terms depending explicitly on finite positron density. The same approach has also been used 
by others (Saito and Oshiyama, 1996; Makhov and Lewis, 2005). The scheme of Gilgien et al. has been shown to predict too localized positron densities due to overestimation of correlation effects (Puska, Seitsonen, and Nieminen, 1995). It may even lead to an unphysical self-trapping phenomenon in a defect-free crystal (Gilgien et al., 1994).

The above non-self-consistent method appropriate for delocalized positrons (the positron does not affect the average electron density, and the zero-positron-density limit of the electron-positron correlation energy used) is routinely applied also for localized positrons. Because of compensation and feedback effects [see Puska, Seitsonen, and Nieminen (1995)] the two-component approach by Boroński and Nieminen and the above so-called "conventional scheme" give rather similar results regarding the positron lifetime and momentum density of annihilating electron-positron pairs. Also the energetics, most importantly the relaxations of vacancy defects in the presence of a localized positron, is described in a similar way.

During its mean lifetime on the order of 150 ps or more, a trapped positron has time to influence its surrounding ionic structure by repelling neighboring ions. When modeling trapped positrons, the detailed atomic structure of the defect affects the resulting positron annihilation parameters. As a first approximation one can use "ideal" unrelaxed geometries where atoms reside on their sites in the pristine lattice. Defect structures relaxed using one-component DFT calculations are not necessarily more accurate since the effect of a localized positron can change the direction of the displacements of the ions neighboring a vacancy from inward to outward. The force on ion $j$ can be calculated within the Born-Oppenheimer approximation from the total derivative of the total energy with respect to ionic position $\mathbf{R}_{j}$ as $\mathbf{F}_{j}=-\nabla_{\mathbf{R}_{j}} E_{\text {tot }}$. In the above conventional scheme with no self-consistency between the electrons and the positrons, the ground-state total energy can be rewritten in the form (Laasonen et al., 1991)

$$
E_{\mathrm{tot}}=E+\varepsilon_{+} \text {, }
$$

where $E$ is the energy of the electron-ion system from the one-component DFT calculation, and $\varepsilon_{+}$is the positron's lowest energy eigenvalue corresponding to the potential of Eq. (39). The positron's contribution to the force on ion $j$ can then be calculated with the Hellmann-Feynman theorem as

$$
\begin{aligned}
\mathbf{F}_{j}^{+}=-\nabla_{\mathbf{R}_{j}} \varepsilon_{+} & =-\nabla_{\mathbf{R}_{j}}\left\langle\psi_{+}\left|H_{+}\right| \psi_{+}\right\rangle \\
& =-\left\langle\psi_{+}\left|\left(\nabla_{\mathbf{R}_{j}} H_{+}\right)\right| \psi_{+}\right\rangle \\
& =\int d \mathbf{r} n_{+}(\mathbf{r})\left[-\nabla_{\mathbf{R}_{j}} V_{+}(\mathbf{r})\right],
\end{aligned}
$$

where $\psi_{+}(\mathbf{r}), H_{+}$, and $V_{+}(\mathbf{r})$ are the positron state and the positron's single-particle Hamiltonian, and the potential, respectively. Here it is assumed that the positron wave function is normalized and that the basis does not depend explicitly on the atomic positions.

The Hellmann-Feynman theorem [Eq. (41)] can also be understood in the following manner. Once the electron and positron densities are calculated quantum mechanically, forces on ions are determined by classical electrostatics.
Therefore, the trapped positron exerts a repulsive force on the neighboring ions.

When the positron is delocalized in a crystal its energy band is parabolic with an effective mass close to the freeelectron mass (Bergersen and Pajanne, 1969). For a single positron in the crystal it is enough to consider only the minimum-energy point $\Gamma(\mathbf{k}=\mathbf{0})$ in the Brillouin zone. When using the supercell approximation to describe a positron localized at an isolated vacancy defect, the positron's discrete energy state is broadened to a narrow energy band due to the finite size of the supercell. For a positron at the $\Gamma$ point, the derivative of the positron's wave function vanishes at the cell boundaries between adjacent vacancies, while the wave function itself has a finite amplitude there. As the wave function is normalized to unity within the supercell, the positron density becomes consequently too high at the boundaries and too low at the vacancy center. In other words, the positron density is too delocalized when the supercell is not large enough. This has been addressed by Korhonen, Puska, and Nieminen (1996). Their remedy is to use two $\mathbf{k}$ points in the Brillouin zone, the $\Gamma$ point and a point from the edge of the Brillouin zone of the superlattice at the top of the energy band. The latter choice implies a boundary condition, which requires the positron wave function to vanish at the supercell boundary. This enables a much faster convergence of the positron density and positron lifetime as a function of the supercell size. Similar ideas have been presented for minimizing interactions between adjacent cells in supercell calculations of aperiodic systems (Makov, Shah, and Payne, 1996). For shallow positron traps one should always check whether the positron density localizes at all using a large supercell and the $\Gamma$ point only. Namely, the scheme by Korhonen et al. can also lead to positron localization for systems where this should actually not happen, namely, when the potential positron trap is shallow and the dispersion of the positron's energy band becomes similar to that in a perfect crystal.

\section{Positron annihilation parameters}

\section{Annihilation rate and lifetime}

Once the electron and positron densities are solved the positron annihilation rate $\lambda$ and mean lifetime $\tau$ can be calculated as (Boroński and Nieminen, 1986)

$$
\lambda=\frac{1}{\tau}=\pi r_{e}^{2} c \int d \mathbf{r} n_{+}(\mathbf{r}) n_{-}(\mathbf{r}) g\left(\mathbf{r}, \mathbf{r} ;\left[n_{+}, n_{-}\right]\right),
$$

where $r_{e}$ is the classical radius of the electron and $c$ is the speed of light. ${ }^{3}$ Here $g\left(\mathbf{r}, \mathbf{r} ;\left[n_{+}, n_{-}\right]\right)$is the so-called enhancement factor, the value of the electron-positron pair correlation function $g\left(\mathbf{r}, \mathbf{r}^{\prime} ;\left[n_{+}, n_{-}\right]\right)$at zero distance. The enhancement factor takes into account the short-range screening of the positron by electrons not accounted for in the average one-body densities $n_{-}(\mathbf{r})$ and $n_{+}(\mathbf{r})$.

\footnotetext{
${ }^{3}$ This is the conventional way to express the prefactor in the positron literature. It can be related to the fine structure constant $\alpha$ by noting that $r_{e}=\alpha^{2}$ (in atomic units).
} 
The electron-positron correlation energy of the twocomponent DFT is related to the electron-positron pair distribution function via (Jensen and Walker, 1988)

$$
\begin{aligned}
E_{c}^{e-p}\left[n_{+}, n_{-}\right]= & -\int_{0}^{1} d \lambda \int d \mathbf{r} d \mathbf{r}^{\prime} \\
& \times \frac{n_{-}(\mathbf{r}) n_{+}\left(\mathbf{r}^{\prime}\right)\left\{g\left(\mathbf{r}, \mathbf{r}^{\prime} ;\left[n_{+}, n_{-}\right], \lambda\right)-1\right\}}{\left|\mathbf{r}-\mathbf{r}^{\prime}\right|},
\end{aligned}
$$

but usually the correlation energy and the enhancement factor are parametrized separately. The electron-positron correlation energy is essentially the Coulomb interaction energy of the positron with its coupling-constant-averaged screening electron cloud. In Eq. (43), $\lambda$ is the coupling constant scaling the electron-positron interaction between noninteracting $(\lambda=0)$ and interacting $(\lambda=1)$ limits, but the densities correspond to the full interaction case.

Typically, also in the case of localized positrons when using the conventional scheme, the enhancement factor is approximated within the LDA and evaluated at the zeropositron-density limit appropriate for delocalized positrons. Then the annihilation rate reads (Chakraborty, 1981; Boroński and Nieminen, 1986; Jensen, 1989)

$$
\lambda=\pi r_{e}^{2} c \int d \mathbf{r} n_{+}(\mathbf{r}) n_{-}(\mathbf{r}) \gamma\left(n_{-}(\mathbf{r})\right),
$$

where $\gamma\left(n_{-}(\mathbf{r})\right)$ is the zero-positron-density limit of the LDA enhancement factor.

\section{Momentum density of annihilating electron-positron pairs}

The quantity measured in Doppler broadening and ACAR experiments is the momentum density of annihilating electron-positron pairs $\rho(\mathbf{p})$. Its integral gives the total annihilation rate,

$$
\lambda=\frac{1}{(2 \pi)^{3}} \int d \mathbf{p} \rho(\mathbf{p}) .
$$

The experiments measure only certain projections of the momentum density. Doppler broadening and onedimensional angular correlation experiments measure onedimensional projections of the momentum density. In a Doppler broadening experiment, the detected energy shift $\Delta E$ is related to the longitudinal momentum component $p_{L}$ of the annihilating pair as $\Delta E=p_{L} c / 2$. Angular correlation experiments measure the deviation of the photons from $180^{\circ}$ by the small angle $\theta=p_{T} / m_{0} c$. Here $m_{0}$ is the electron mass and $p_{T}$ is the measured transverse momentum component. The one-dimensional projection of the momentum density detected by these techniques can be written as

$$
\rho\left(p_{z}\right)=\iint d p_{x} d p_{y} \rho(\mathbf{p})
$$

where $p_{z}$ is the measured momentum component $\left(p_{L}\right.$ or $p_{T}$ depending on the technique), and $p_{y}$ and $p_{y}$ are the corresponding transverse components. On the other hand, two-dimensional angular correlation measurements measure a two-dimensional projection,

$$
\rho\left(p_{x}, p_{y}\right)=\int d p_{z} \rho(\mathbf{p}) \text {. }
$$

When comparing measured spectra (Doppler, 1D-ACAR, 2D-ACAR) with corresponding computational ones, one first has to either convolute the theoretical ones with the experimental resolution function or correspondingly deconvolute the experimental ones.

Electronic-structure calculations done within DFT provide direct access only to the one-body densities, not to the true many-body wave function of the interacting system. Strictly speaking, this or at least the relevant reduced two-body electron-positron density matrix would be required to calculate the momentum density of annihilating electron-positron pairs. A formal but unfortunately impractical relation exists for expressing general operator ground-state expectation values including momentum densities of the interacting system within the Kohn-Sham density-functional formalism (Bauer, 1983; Barbiellini et al., 1999). This theorem is a generalization of the Lam-Platzman correlation correction routinely used in x-ray Compton scattering (Lam and Platzman, 1974).

For a noninteracting system the momentum density reads

$$
\rho(\mathbf{p})=\pi r_{e}^{2} c \sum_{j}\left|\int d \mathbf{r} e^{-i \mathbf{p} \cdot \mathbf{r}} \psi_{+}(\mathbf{r}) \psi_{j}(\mathbf{r})\right|^{2} .
$$

Here and in the models discussed below, the summation is over occupied single-particle orbitals. This is the so-called independent-particle model (IPM) neglecting effects of shortrange electron-positron correlations, i.e., the screening of the positron by electrons. There exist a number of improved approximations for calculating the momentum density of annihilating electron-positron pairs using the single-particle orbitals of the noninteracting Kohn-Sham system. A general expression for periodic systems typically applied within the LDA can be written as (Šb, Sormann, and Kuriplach, 2003)

$$
\begin{aligned}
\rho(\mathbf{p})= & \sum_{j \mathbf{k}} \delta(\mathbf{p}-\mathbf{k}-\mathbf{G}) \\
& \times\left|\int d \mathbf{r} e^{-i \mathbf{p} \cdot \mathbf{r}} \psi_{+}(\mathbf{r}) \psi_{j \mathbf{k}}(\mathbf{r}) \sqrt{g_{e p}(\mathbf{r} ; j \mathbf{k})}\right|^{2},
\end{aligned}
$$

where $\mathbf{k}$ is the crystal momentum of the electron's Bloch state, $\mathbf{G}$ is a reciprocal lattice vector, and $g_{e p}(\mathbf{r} ; j \mathbf{k})$ is a twoparticle enhancement function depending on the position $\mathbf{r}, \mathbf{k}$, and band index $j$. In defect studies with coincidence Doppler broadening spectroscopy, in which the most important signal is often contained at high momenta and is due to core electron annihilation and umklapp components $(\mathbf{G} \neq \mathbf{0})$ of valence electrons, the correct description of the $\mathbf{k}$ dependence of the enhancement is less important than in Fermi surface studies with 2D-ACAR [for reviews on the topic and discussion see, for e.g., Mijnarends and Bansil (1993), West (1993), Kontrym-Sznajd (2009), Laverock et al. (2010), and Kontrym-Sznajd, Sormann, and Boroński (2012)]. More important is how well the core electron annihilation and the $\mathbf{G}$ dependence of the enhancement are described. Most models used do not involve any explicit $\mathbf{k}$ dependence at all.

Šob (1978, 1979), and Mijnarends and Singru (1979) parametrized a phenomenological momentum-dependent enhancement factor based on the electron gas results of Kahana (1960, 1963). They write the enhancement in terms of energy instead of the momentum by equating $p / p_{F}=\sqrt{E_{j \mathbf{k}} / E_{F}}$, where $E_{j \mathbf{k}}$ is the one-particle energy of an electron in the 
$j$ th band at Bloch vector $\mathbf{k}$ measured from the bottom of the conduction band, and $p_{F}$ and $E_{F}$ are the Fermi momentum and Fermi energy, respectively. Below, in the context of models without explicit $\mathbf{k}$ dependence, we drop this index for simplicity.

Daniuk et al. (1987) and Jarlborg and Singh (1987) parametrized position-dependent LDA enhancement factors incorporating effects of short-range electron-positron correlations,

$$
\rho(\mathbf{p})=\pi r_{e}^{2} c \sum_{j}\left|\int d \mathbf{r} e^{-i \mathbf{p} \cdot \mathbf{r}} \psi_{+}(\mathbf{r}) \psi_{j}(\mathbf{r}) \sqrt{\gamma\left(n_{-}(\mathbf{r})\right)}\right|^{2} .
$$

A similar approach has also been used for core states (Daniuk et al., 1989; Daniuk, Šob, and Rubaszek, 1991; Alatalo et al., 1995).

A state-dependent enhancement factor was parametrized and used to describe core electron annihilation by Alatalo et al. (1996) and later applied also for valence electrons (Barbiellini et al., 1997)

$$
\rho(\mathbf{p})=\pi r_{e}^{2} c \sum_{j} \gamma_{j}\left|\int d \mathbf{r} e^{-i \mathbf{p} \cdot \mathbf{r}} \psi_{+}(\mathbf{r}) \psi_{j}(\mathbf{r})\right|^{2} .
$$

Here $\gamma_{j}=\lambda_{j}^{\mathrm{LDA}} / \lambda_{j}^{\mathrm{IPM}}$, where

$$
\lambda_{j}^{\mathrm{LDA}}=\pi r_{e}^{2} c \int d \mathbf{r} n_{+}(\mathbf{r})\left|\psi_{j}(\mathbf{r})\right|^{2} \gamma\left(n_{-}(\mathbf{r})\right),
$$

and $\lambda_{j}^{\mathrm{IPM}}$ is calculated similarly with $\gamma \equiv 1$. The scheme can be applied instead of the LDA equally well within the GGA for elecron-positron systems (see Sec. III.D). Recently, the empirical model of Laverock et al. (2010) combined the above model of Alatalo et al. with an energy-dependent enhancement factor.

Tang et al. (2005) also applied together with Eq. (50) within the LDA of Puska, Seitsonen, and Nieminen (1995) the $G W$ self-energy correction to correct electron occupation numbers.

\section{Functionals for electron-positron correlation effects}

The true many-body interactions responsible for the short-range screening of the positron by the electrons are taken into account in the two-component DFT only in an approximate manner. One takes input from many-body calculations made for electron-positron systems and parametrizes the unknown functionals, the electron-positron correlation energy $E_{c}^{e-p}\left[n_{-}, n_{+}\right]$, and the enhancement factor $g\left(\mathbf{r}, \mathbf{r} ;\left[n_{+}, n_{-}\right]\right)$, which incorporate the effects of the screening into the energy functional and on the positron annihilation rate. The starting point is usually the LDA, in which the quantities are approximated using functions of the local electron and positron densities. The electron-positron correlation energy within the LDA is then written with the help of the correlation energy density per unit volume $F_{c}^{e-p}\left(n_{-}, n_{+}\right)$, in a homogenous electron-positron gas with densities $n_{-}$and $n_{+}$(Boroński and Nieminen, 1986; Puska, Seitsonen, and Nieminen, 1995)

$$
E_{c}^{e^{-} p}\left[n_{-}, n_{+}\right]=\int d \mathbf{r} F_{c}^{e^{-} p}\left(n_{-}(\mathbf{r}), n_{+}(\mathbf{r})\right)
$$

i.e., assuming that at each point in space the local correlation energy density is equal to that in a homogenous system with densities equal to the local ones. Similarly, the finite-positrondensity LDA enhancement factor $\gamma\left(n_{-}(\mathbf{r}), n_{+}(\mathbf{r})\right)$ is taken from results for homogenous systems. In the zero-positrondensity limit, the LDA correlation energy can be expressed using the correlation energy per particle as in the case of one-component DFT (Boroński and Nieminen, 1986),

$$
E_{c}^{e^{-} p}\left[n_{-}, n_{+} \rightarrow 0\right]=\int d \mathbf{r} n_{+}(\mathbf{r}) \varepsilon_{c}^{e-p}\left(n_{-}(\mathbf{r}), n_{+} \rightarrow 0\right),
$$

where $\varepsilon_{c}^{e-p}\left(n_{-}, n_{+} \rightarrow 0\right)$ is the correlation energy per positron in a homogenous electron-positron gas with electron density $n_{-}$and a single delocalized positron. In this limit, the LDA enhancement factor becomes simply a function of the local electron density $\gamma(n(\mathbf{r}))$; see Eq. (44).

For the case of finite densities, there exist LDA parametrizations by Boroński and Nieminen (1986) and Puska, Seitsonen, and Nieminen (1995). Both are based on multicomponent Fermi hypernetted-chain calculations by Lantto (1987), and the zero-positron-density limit calculations of Arponen and Pajanne (1979a, 1979b). However, Boroński and Nieminen had only the data for $n_{+} \rightarrow 0, n_{+}=n_{-} / 2$, and $n_{+}=n_{-}$available. The zero-positron-density limit has been parametrized by Boroński and Nieminen (1986) using correlation energy from Arponen and Pajanne (1979a, 1979b) and the contact density of Lantto (1987). The calculations of Arponen and Pajanne are based on correcting the results of the random-phase approximation in a boson formalism. Barbiellini et al. (1995, 1996) reparametrized the LDA enhancement factor to be consistent with the data by Arponen and Pajanne and used this parametrization as a basis for their gradient corrections. Recently, Drummond et al. (2011) parametrized a LDA for correlation energy and enhancement factor using their own quantum Monte Carlo results. This work followed an earlier one by the same group (Drummond et al., 2010) in which the screening of the positron was modeled by applying one-component DFT in the positron's reference frame.

Before the time of $a b$ initio positron lifetime calculations, enhancement models were empirical, based on dividing the total electron density $n(\mathbf{r})$ into core $n_{c}(\mathbf{r})$ and valence $n_{v}(\mathbf{r})$ components, and, when applicable, to a $d$ electron component $n_{d}(\mathbf{r})$ and using a different enhancement factor for each of these [see, e.g., Puska and Nieminen (1983)]. Puska and Nieminen used a constant enhancement factor for core electrons, and the $d$ electron enhancement factors were determined by fitting to measured bulk lifetime data. Jensen (1989) was the first to evaluate the positron annihilation rate using the LDA of Eq. (44), using the total density and without adjustable parameters.

Most models describing electron-positron correlation energy and enhancement effects derive information from many-body modeling made for metallic systems, i.e., the homogenous electron-positron gas. The screening of the positrons in semiconductors and insulators with less efficient screening is less understood. Puska et al. (1989) introduced semiempirical models for semiconductors and insulators. The parameter in the semiempirical semiconductor model, based on the earlier work of Brandt and Reinheimer (1970), is the 
high-frequency dielectric constant, whereas in the insulator model the enhancement is connected to the atomic polarizability. Previously a model describing the screening of positive point charges in semiconductors (Brandt and Reinheimer, 1971) had been adapted to describe electronpositron correlations (Puska et al., 1986). In this case the free parameter in the model was fitted to reproduce the experimental bulk lifetime.

Barbiellini et al. $(1995,1996)$ approached the correlation problem by adding gradient corrections to the LDA correlation energy and enhancement factor. Their approach involves one semiempirical parameter chosen by requiring good agreement between calculated and measured positron lifetimes for a large number of different solids. For predicting absolute values of positron lifetimes this approach is presently the most accurate one [see, e.g., Campillo Robles, Ogando, and Plazaola (2007)], although it has been shown that it might need further improvements in the very lowdensity regime (Mitroy and Barbiellini, 2002). Analysis of the behavior of this GGA model helped to further explain why the annihilation rates for core electrons can be well described by the independent-particle limit [see, e.g., Alatalo et al. (1996)].

A completely nonlocal model based on the original work by Gunnarsson, Jonson, and Lundqvist (1979) is the weighted-density approximation (WDA) formulated for the electron-positron case by Jensen and Walker (1988) and Rubaszek (1991) and applied for positron surface states. More recent applications on bulk solids include Rubaszek, Szotek, and Temmerman (1998, 2000, 2001, 2002). The WDA has not, however, been applied for positrons trapped at vacancy defects.

\section{E. The atomic superposition method}

The so-called atomic superposition (ATSUP) method (Puska and Nieminen, 1983) is a simple but already very applicable method for modeling positron states and annihilation in solids. In essence, the method is based on a fully non-self-consistent approach to the two-component DFT presented above. The electron density is approximated as a superposition of densities of neutral free atoms,

$$
n_{-}(\mathbf{r}) \approx \sum_{\mathbf{R}} n_{-}^{\text {at }}(|\mathbf{r}-\mathbf{R}|),
$$

and the positron's potential is a sum of Coulomb potentials due to the superimposed free atoms and a LDA correlation potential similar to that in Eq. (39),

$$
V_{+}(\mathbf{r}) \approx \sum_{\mathbf{R}} V_{\text {Coul }}^{\text {at }}(|\mathbf{r}-\mathbf{R}|)+V_{\text {corr }}\left(n_{-}(\mathbf{r})\right) \text {. }
$$

Positron lifetimes calculated with the atomic superposition method agree remarkably well with those calculated with more self-consistent methods [see, e.g., Puska et al., 1986; Puska, 1991; Campillo Robles, Ogando, and Plazaola, 2007], even within a few picoseconds, as long as lattice parameters and ionic positions are the same and the same functionals are used for the correlation energy and enhancement factor. This is due to a simple compensating feedback effect (Puska and Nieminen, 1983, 1994). The positron density will follow any changes in the electron density, for example, due to an improved description, keeping their mutual overlap, the annihilation rate [see Eq. (42)], constant.

First calculations of coincidence Doppler broadening spectra were done within the ATSUP method (Alatalo et al., 1995, 1996; Asoka-Kumar et al., 1996). The results of the method are rather accurate at high momenta dominated by the core shells, which are rigid and do not depend much on the chemical environment of the atoms. When modeling Doppler broadening one typically assumes in addition to the spherical symmetry of the core electron shells the positron wave function to be spherically symmetric around nuclei. Its decay toward the nucleus is parametrized using all-electron calculations done, for example, with the linear-muffin-tin-orbital method (Alatalo et al., 1995, 1996). The same approach can be used when including the core electron contribution in calculations employing the frozen-core approximation, such as the plane-wave pseudopotential method or the projectoraugmented-wave method (see Sec. III.F).

The atomic superposition method is computationally cheap and it still remains an applicable method also in modeling Doppler broadening as long as results are compared with experiments only at high momenta [ $>(2-2.5)$ a.u.] dominated by core electron annihilation. For instance, the MIKA Doppler software package (Torsti et al., 2003, 2006) implements calculations with the atomic superposition method and provides also the possibility of coupling the code with DFT codes in order to perform more self-consistent calculations with accurate electron densities.

\section{F. Numerical approaches for self-consistent calculations}

Practical modeling of positron states and annihilation in solids going beyond the atomic superposition method (Puska and Nieminen, 1983, Sec. III.E), positron pseudopotential (Kubica and Stott, 1974; Stott and Kubica, 1975), or jellium models [see, e.g., Manninen et al. (1975) and Boroński and Nieminen (1986)] is typically based on some standard bandstructure method for performing the underlying electronicstructure calculation. Depending on the level of sophistication and self-consistency, the positron is either treated within the same code or added in the system in a postprocessing manner (see the end of Sec. III.A). The numerical representation of the positron wave function is in a sense less complicated than those of electrons, since in the typical case of only one positron in the lattice there are no orthogonality requirements, and the wave function is of $s$ type, soft and nodeless. On the other hand, the basis used for the positron has to work well in regions in which the positron wave function has the largest amplitude, such as interstitial regions and vacancies. In non-all-electron methods where the frozen-core approximation is used, such as in planewave pseudopotential calculations, the annihilation with core electrons typically is described using frozen-core densities and orbitals, and when modeling momentum densities of annihilating electron-positron pairs, a spherically symmetric approximation for the positron wave function is used. Periodic boundary conditions and the supercell approximation are used in most numerical implementations also when one is modeling positrons trapped at isolated vacancy-type defects. Figure 9 shows an example of a model for a Ga vacancy in a 96-atom 


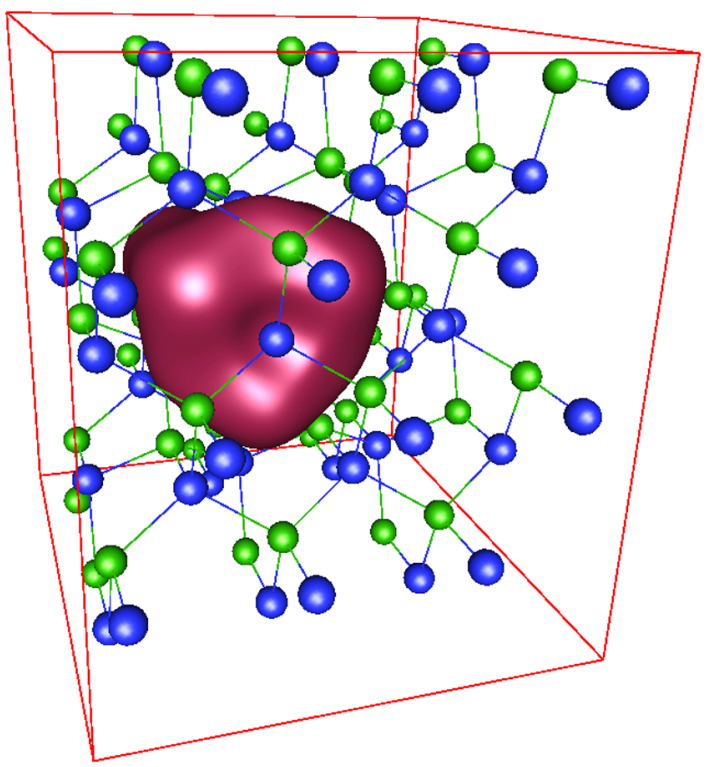

FIG. 9 (color online). An isosurface of the positron density for a positron localized at a Ga vacancy in GaN in a 96-atom supercell model. The $\mathrm{Ga}$ atoms are shown by light shaded and the $\mathrm{N}$ atoms by dark shaded spheres. Based on the calculations of Hautakangas et al. (2006). From Torsti et al., 2006.

GaN supercell used by Hautakangas et al. (2006). Care has to always be taken so that the results are converged with respect to the size of the supercell. A ground state with a localized positron can be confirmed only by visualization of the realspace positron density $n_{+}(\mathbf{r})$ as in Fig. 9. A lifetime increase compared to the bulk value is not a safe indicator alone.

Here we list some band-structure methods which have been applied to model positrons states and annihilation in solids. We mostly focus on the so-called projector-augmented-wave method (Blöchl, 1994), which currently is the most useful one when modeling coincidence Doppler broadening spectra for positrons localized at defects in solids.

Berko and Plaskett (1958) used the Wigner-Seitz bandstructure method for describing positron annihilation in metals.

The augmented plane-wave (APW) method by Slater (1937) was used by several groups (Loucks, 1966; Wakoh, Kubo, and Yamashita, 1975; Kubo, Wakoh, and Yamashita, 1976; Gupta and Siegel, 1977, 1980a, 1980b) in a non-selfconsistent fashion. The supercell approximation was used for localized positrons first by Gupta and Siegel (1977). Also the linearized (LAPW) (Andersen, 1975) (Daniuk, 1983; Daniuk et al., 1987) and the full-potential version (FP-LAPW) (Baruah, Zope, and Kshirsagar, 1999; Tang, Hasegawa, Nagai, and Saito, 2002; Tang, Hasegawa, Nagai, Saito, and Kawazoe, 2002) have been applied. The FP-LAPW method is highly accurate and treats also the core electrons and their annihilation fully self-consistently using the true symmetry of electron and positron states. However, we are not aware of any calculations made for localized positrons with the FP-LAPW method.

The linear-muffin-tin orbital (LMTO) method (Andersen, 1975 ) is another all-electron method which has been widely used in the positron community, in both defect identification and Fermiology applications [for bulk studies see, e.g., Singh and Jarlborg (1985), Jarlborg and Singh (1987), Sterne and Kaiser (1991), and Barbiellini, Dugdale, and Jarlborg (2003)]. Positrons localized at vacancies have been studied (Puska et al., 1989, 1994; Puska, 1991; Alatalo, Puska, and Nieminen, 1993; Plazaola, Seitsonen, and Puska, 1994; Korhonen, Puska, and Nieminen, 1996; Barbiellini et al., 1997), also using the LMTO Green's function method (Puska et al., 1986). In most of the works the so-called atomic-sphere approximation (Skriver, 1984; Andersen, Jepsen, and Glötzel, 1985) has been made but also the full-potential variant has been used (Korhonen, Puska, and Nieminen, 1996).

The Korringa-Kohn-Rostoker (KKR) Green's function method (Korringa, 1947; Kohn and Rostoker, 1954) has also been applied in bulk studies (Hanssen and Mijnarends, 1986; Mijnarends and Rabou, 1986; Bansil et al., 1988; Mijnarends and Bansil, 1990; Bansil, Mijnarends, and Smedskjaer, 1991; Mijnarends et al., 1998; Eijt et al., 2006), and within the coherent potential approximation (CPA) (Soven, 1967, 1969; Velický, Kirkpatrick, and Ehrenreich, 1968) for alloys (Mijnarends et al., 1987; Smedskjaer et al., 1987). Further, some of the more approximate methods used earlier (Hubbard, 1969; Hubbard and Mijnarends, 1972), for example, by Mijnarends (1973), Singru and Mijnarends (1974), and Mijnarends and Singru (1979) were based on the KKR method.

Ishibashi et al. (1997) used localized orbitals in allelectron calculations. The positron state was solved on a grid using finite differences.

Finite-difference and finite-element methods are very useful in positron calculations, since the region where accuracy is needed is different between electrons and the positron. Whereas electronic orbitals oscillate rapidly close to nuclei, from the positron's point of view this region is unimportant because of the vanishingly small positron density there. More important are the less repulsive regions in the interstitial space and open-volume defects.

Puska and Nieminen (1983) used in the first atomic superposition calculations the finite-difference method of Kimball and Shortley (1934). The finite-difference method has also been used with the conjugate-gradient solver (Seitsonen, Puska, and Nieminen, 1995) and the Rayleigh quotient multigrid method (Heiskanen et al., 2001); see Torsti et al. (2003, 2006). Pask et al. (1999, 2001) and Sterne, Pask, and Klein (1999) used finite-element modeling for electron-positron systems.

Especially when modeling positrons trapped in vacancy defects it becomes necessary to use rather large supercells with even hundreds of atoms and relax the defect structures modeled. For these purposes, the method of choice has in total energy calculations traditionally been the plane-wave pseudopotential method with either norm-conserving [see, e.g., Hamann, Schlüter, and Chiang (1979), Kerker (1980), Bachelet, Hamann, and Schlüter (1982), Vanderbilt (1985), and Troullier and Martins (1991)] or ultrasoft pseudopotentials (Blöchl, 1990; Vanderbilt, 1990), or lately especially the closely related all-electron technique, the projector-augmented-wave (PAW) method (Blöchl, 1994), discussed in more detail in the next paragraph. From the point of view of positron defect calculations these methods also bring flexibility. Also, they do not involve any other shape approximations 
than the one of spherically symmetric ion cores inherent in the frozen-core approximation. Plane-wave pseudopotential methods have been used extensively both within the framework of Car and Parrinello (1985) [see, e.g., Laasonen et al. (1991), Alatalo, Puska, and Nieminen (1993), Gilgien et al. (1994), and Puska, Seitsonen, and Nieminen (1995)] and/or in other Born-Oppenheimer calculations (Hakala, Puska, and Nieminen, 1998; Ishibashi et al., 1999; Saarinen et al., 1999; Ishibashi and Kohyama, 2003). The downside related to momentum-density calculations is the inaccuracy of the high-momentum components of valence wave functions.

The increasing sophistication of the plane-wave pseudopotential method has led to better computational accuracy with smaller computational cost and smaller plane-wave bases. However, in order to accurately describe the wave function's high-momentum components, which are essential in defect identification, a small plane-wave basis is not enough. To exemplify, the coincidence Doppler broadening measurements can measure the Doppler spectra accurately up to a momentum of 6-8 a.u. Since the Doppler spectrum is a one-dimensional projection of the three-dimensional momentum density of annihilating electron-positron pairs, an even higher momentum-space cutoff on the order of 10 a.u. is needed. This corresponds to a plane-wave kinetic energy cutoff of $\sim 1400 \mathrm{eV}$ and implies high requirements for the numerical accuracy of the high-momentum plane-wave components. Calculations with such high cutoffs (cf. typical values of $250-400 \mathrm{eV}$ ) are not affordable. Further, since the pseudo wave functions are "softened," they do not represent the accurate all-electron wave functions even in the limit of a complete basis set. Techniques exist to reconstruct the allelectron wave functions from norm-conserving or ultrasoft wave functions (Meyer et al., 1995; Delaney, Králik, and Louie, 1998; Hetényi et al., 2001; Ishibashi, 2004) in a postprocessing fashion. Furthermore, the PAW method is especially useful since it involves a well-defined relation between soft pseudo wave functions $|\tilde{\Psi}\rangle$ represented in a plane-wave basis and the accurate all-electron wave functions $|\Psi\rangle$ (Blöchl, 1994),

$$
|\Psi\rangle=|\tilde{\Psi}\rangle+\sum_{i}\left(\left|\phi_{i}\right\rangle-\left|\tilde{\phi}_{i}\right\rangle\right)\left\langle\tilde{p}_{i} \mid \tilde{\Psi}\right\rangle .
$$

Here $\left|\phi_{i}\right\rangle,\left|\tilde{\phi}_{i}\right\rangle$, and $\left|\tilde{p}_{i}\right\rangle$ are atom-centered all-electron and pseudo partial waves and projector functions, respectively. The index $i$ is a shorthand index referring to the atomic site, angular momentum quantum numbers, and reference energy. Equation (57) is applied as a postcorrection after a selfconsistent calculation made using effectively the soft pseudo wave functions $|\tilde{\Psi}\rangle$, either on a dense real-space grid or in momentum space using an expanded plane-wave basis (Ishibashi, 2004; Makkonen, Hakala, and Puska, 2005, 2006). In the case of positrons localized at vacancies the transformation needs to be applied only within the first few coordination shells around the vacancy. Since the positron wave function is smooth, it can be represented using only a plane-wave expansion or a real-space point grid. In this approach, an accurate enough numerical potential for the positron is obtained from, instead of the all-electron density never calculated explicitly within the method, a sum of the core density, pseudovalence charge density, and atom-centered compensation charges of the PAW method (Makkonen, Hakala, and Puska, 2006).

Positron lifetimes or momentum densities of annihilating electron-positron pairs have been calculated using PAW implementations based on such modern DFT packages as the quantum materials simulator (QMAS) (Ishibashi, 2004; Ishibashi et al., 2007), Vienna ab initio simulation package (VASP) (Kresse and Hafner, 1993; Kresse and Furthmüller, 1996a, 1996b; Kresse and Joubert, 1999) [see, e.g., Makkonen, Hakala, and Puska, 2005, 2006], and ABINIT (Gonze et al., 2009; Wiktor et al., 2013).

\section{RESULTS}

In this section, we present a series of examples showing how the combination of positron lifetime experiments, regular and coincidence Doppler broadening experiments, sample state manipulation, and theoretical calculations has been used to identify and elucidate properties of defects in semiconductors. As an introduction, we review the types of studies performed and results obtained with positron annihilation methods on defects in various semiconductor material families over the past 20 years of active research in this field. The core of this section then consists of a detailed account of seven cases: (i) vacancy-(multi-)donor complexes in silicon, (ii) the vacancy-fluorine complex in silicon and silicongermanium alloys, (iii) the "EL2" defect in gallium arsenide, (iv) the gallium vacancy-tellurium complex in gallium arsenide, (v) the gallium vacancy and its complexes in gallium nitride, (vi) metal vacancy-nitrogen vacancy complexes in III-nitrides and their alloys, and (vii) the substitutional lithium-on-zinc-site defect in zinc oxide. These cases have been chosen in order to illustrate different approaches and features observed in the experimental spectra. We also discuss the physical properties and importance of each of the above defects, relating the positron results to other experimental and theoretical findings. Future challenges are discussed in the next section.

\section{A. An overview of results obtained in the past two decades}

The examples given in the next sections concentrate on cases where a detailed identification of the vacancy-type defects has provided a basis for further work. In the majority of situations, however, detailed identification of the atomic structure of the defects observed with positrons is not possible. The two most typical cases are (i) the presence of multiple kinds of (vacancy) defects with high concentrations, and (ii) the inability to define a proper reference (i.e., "defect-free" sample). These situations often coexist, as they tend to be related to the challenges in material growth, e.g., typical of early stages of a development of a new semiconductor. Another issue is that many semiconductors can be grown only as thin films, as is the case for the majority of alloyed systems such as $\mathrm{Si}_{1-x} \mathrm{Ge}_{x}$ or $\mathrm{Al}_{1-x} \mathrm{Ga}_{x} \mathrm{As}$. Also some compounds, such as InN, cannot be found as bulk crystals (substrates). Hence, lifetime measurements for finding a suitable reference sample are complicated. In addition, epitaxial growth has to be performed on non-native substrates, increasing the probability of lattice mismatch and ensuing high extended defect densities. Luckily, insightful 
studies on the formation of defects and on their properties can be performed even without detailed identification.

Typical studies, where it is enough to know the vacancy character (sublattice in compounds) and size, concentrate on the effects of growth parameters on the vacancy formation. Such parameters can be the growth temperature, stoichiometry, the addition of dopants or surfactants, or the choice and processing of the substrate or buffer layer. Thermal formation of vacancies can be studied through either analyzing growth data or postgrowth thermal annealings. The latter can be used for studying the migration kinetics of in-grown defects, but these kinds of studies are more convenient in irradiated or ion-implanted samples, where defects are often better defined. Defects introduced in ion implantation processing are by themselves an important field of study, as they dramatically affect the doping efficiency achieved by implantation. High-energy electron irradiation is of particular use when controlled defect introduction is needed, and it often provides a baseline for defect identification.

\section{Elemental semiconductors $\mathrm{Si}, \mathrm{Ge}$, and $\mathrm{C}$}

The formation enthalpy of a monovacancy in $\mathrm{Si}$ is predicted to be rather high, above $3 \mathrm{eV}$ (Puska et al., 1998; Probert and Payne, 2003) for all charge states and Fermi level positions within the band gap. This prediction is in good agreement with experimental findings from both diffusion (Bracht et al., 2003) and positron annihilation (Ranki and Saarinen, 2004; Kuitunen, Saarinen, and Tuomisto, 2007) studies where thermal formation of vacancies requires rather high temperatures, even when the vacancies are formed right next to donors in highly $n$-type material. Hence, the vast majority of positron annihilation studies on vacancies in silicon have been performed on irradiated or implanted material, with possibly the only exception being highly $n$-type doped Si (see Sec. IV.B). Irradiation and implantation studies have enabled the identification of the monovacancy (at low temperature) and determination of its migration barrier in good agreement with EPR studies (Mäkinen, Rajainmäki, and Linderoth, 1990; Watkins, 2000). The divacancy can be detected and identified after room-temperature irradiation, and also the optical ionization levels and dissociation energy have been determined (Kauppinen et al., 1998). Larger vacancy clusters have been observed in ion-implanted (Avalos and Dannefaer, 1996) and neutron-irradiated (Meng et al., 1994) material. Complexes of vacancies with impurities, in particular, donors such as As, $\mathrm{Sb}$, or $\mathrm{P}$, have been studied a great deal, as well as the $V-O$ (Kauppinen et al., 1998) and $V$-F systems. Vacancy-donor and vacancy-fluorine complexes are discussed in more detail in Secs. IV.B and IV.C. Vacancies and vacancy clusters in ion implantation processing have been studied mostly from the point of view of affecting the diffusion of either interstitials or boron.

Germanium has been studied less than Si also with positron annihilation spectroscopies, although lately some revival can be seen. Monovacancies and divacancies have been identified (Corbel, Moser, and Stucky, 1985; Polity and Rudolf, 1999; Kuitunen et al., 2008; Slotte et al., 2008, 2011) and their stability at and below room temperature investigated. Vacancies have also been found to pair with donors in $\mathrm{Ge}$ with positron annihilation (Arutyunov and Emtsev, 2007), while thorough investigations are still to be performed. Even though germanium exhibits interesting properties in ion implantation, i.e., it is easily amorphized and the recrystallization temperature is low (Hickey et al., 2007), surprisingly few positron studies seem to have been performed (Krause-Rehberg et al., 1993; Slotte et al., 2008). The situation is completely different for the $\mathrm{Si}-\mathrm{Ge}$ alloys which have been studied to a large extent. Vacancy-donor complexes introduced by irradiation of $n$-type material (Sihto et al., 2003; Rummukainen et al., 2006; Kuitunen, Tuomisto, and Slotte, 2007), vacancy-fluorine complexes produced by F implantation (Edwardson et al., 2012), and the particularities related to the random alloy nature of $\mathrm{Si}-\mathrm{Ge}$ alloys (Shoukri et al., 2005; Ferragut et al., 2010; Kilpeläinen et al., 2010, 2011) have been studied in more detail.

Point defects in diamond have been studied quite actively, but not so much from the semiconducting properties point of view. Studies have concentrated on elucidating the fundamental properties of the crystal such as the mobility of isolated vacancy defects introduced by irradiation (Uedono et al., 1999; Pu et al., 2000; Iakoubovskii, Dannefaer, and Stesmans, 2005; Dannefaer and Iakoubovskii, 2008), or the vacancy-related coloration of natural and synthetic diamond (Nilen et al., 1997; Dannefaer, Pu, and Kerr, 2001; Mäki et al., 2009; Mäki, Tuomisto et al., 2011). Nitrogen-vacancy centers, which have high potential in quantum computing (Maurer et al., 2012) and, in particular, in hypersensitive high-dynamic range magnetometry (Waldherr et al., 2012), have received some attention (Dannefaer, 2009; Botsoa et al., 2011). The formation of vacancy-donor complexes, which are potentially important in limiting the $n$-type dopability of diamond, seems not to have been addressed with positron methods.

\section{Traditional III-V and II-VI semiconductors}

Compound semiconductors have provided a particularly fruitful ground for studying point defects and their effects on the electrical and optical properties of these materials. The formation enthalpies of, e.g., the vacancy defects on both sublattices tend to be clearly lower than in $\mathrm{Si}$, and in addition changes in growth stoichiometry can further increase the probability of defect formation. Further, these defects tend to be stable well above room temperature and they produce a multitude of localized states in the band gap, hence controlling many of the crucial material properties. By traditional III-V and II-VI semiconductors we mean the III-phosphides, III-arsenides, and III-antimonides, and II-selenides and II-tellurides. The body of work with any defect spectroscopy on these materials is large. An important property of the traditional III-V and II-VI compounds is that neither of the constituent atoms is "small," i.e., positrons can be at least in principle trapped by vacancy defects on either sublattice. This is in contrast to the "novel" compound semiconductors, where the anion is typically small (N, O, or C); see Sec. IV.A.3.

The studies of vacancy defects in GaAs range from ingrown and irradiation-induced defects in $n$-type and semiinsulating crystals to the defects formed in low-temperature molecular beam epitaxy (LT-MBE) of thin films. The studies have shown that negatively charged Ga vacancies and their complexes with donors are important compensating centers in 
n-type doped GaAs (Dannefaer, Hogg, and Kerr, 1984; Corbel et al., 1988; Laine et al., 1996; Gebauer et al., 1999). Arsenic vacancies are observed in unintentionally $n$-type as-grown GaAs crystals (Saarinen et al., 1991), while they coexist with Ga vacancies and EL2 defects in semiinsulating GaAs (Kuisma et al., 1996). The metastable EL2 and DX centers in arsenides have both been shown to have vacancy character (Krause, Saarinen et al., 1990; Mäkinen et al., 1993). Thermal annealing experiments have been performed to elucidate vacancy formation mechanisms (Bondarenko et al., 2005) and the role of copper in determining the vacancy distribution in GaAs crystals (Elsayed et al., 2008, 2011). LT-MBE GaAs is typically grown in conditions that favor the formation of Ga sublattice defects: Ga vacancies and As antisites (Bliss et al., 1992; Keeble et al., 1993; Störmer et al., 1996; Fleischer et al., 1997; Gebauer et al., 1997; Laine et al., 1999). The Ga vacancy-related defects follow the growth stoichiometry and compensate the $n$-type doping. Similar effects have been found in the magnetically doped dilute magnetic semiconductor $\mathrm{Ga}_{1-x} \mathrm{Mn}_{x} \mathrm{As}$ (Tuomisto et al., 2004) that is also grown by LT-MBE.

In III-phosphides (such as $\mathrm{GaP}$ or InP) the vacancies on both sublattices have been identified, and these have an important effect on the electrical properties in undoped, $n$-type, and $p$-type material (Mahony, Mascher, and Puff, 1996; Bretagnon, Dannefaer, and Kerr, 1997; Dekker et al., 2002). As an example, the $V_{\mathrm{P}} \mathrm{Zn}$ pairs have been shown to form through vacancy migration from the crystal surface in Zn-doped InP (Slotte et al., 2003), while the In vacancies formed through thermal annealings render undoped InP semiinsulating through compensation of residual donors (Deng et al., 2003). Alloys of III-V semiconductors, such as GaInP (Dekker et al., 2002), AlGaAs (Mäkinen et al., 1993), or InGaAsP (Pinkney et al., 1998) have also been studied to some extent, as well as the so-called diluted nitrides where at most a few percent of nitrogen replaces the group- $\mathrm{V}$ component (Toivonen et al., 2003). Cation vacancies are typically found to be strongly correlated with the optoelectronic properties in these studies. Much of the positron research in III-V semiconductors is covered by Saarinen, Hautojärvi, and Corbel (1998) and Krause-Rehberg and Leipner (1999).

The most important early positron results in II-VI compound semiconductors have been reviewed by KrauseRehberg et al. (1998). The interest in studying defects in these materials stemmed from the doping asymmetry: bulk crystals of $\mathrm{ZnSe}, \mathrm{CdSe}, \mathrm{ZnS}$, and $\mathrm{CdS}$ tend to always be $n$ type, irrespective of impurity content, while ZnTe is $p$ type. The II-VI compounds appeared promising for various device applications ranging from particle detectors to optoelectronic devices; their technological breakthroughs have been limited due to this issue. Only CdTe is easy to dope either way. The situation in thin films is clearly better: e.g., $p$-type $\mathrm{ZnSe}$ can be grown by MBE and hence $p-n$ junctions can be fabricated (Park et al., 1990). Vacancy defects on both sublattices have been shown to exist depending on doping in $\mathrm{ZnS}_{x} \mathrm{Se}_{1-x}$ (Saarinen et al., 1996; Oila et al., 1999; Desgardin et al., 2000; Gebauer et al., 2002). Cation vacancies complexed with impurities in CdTe and $\mathrm{Hg}_{1-x} \mathrm{Cd}_{x} \mathrm{Te}$ have been identified as important defects controlling the conductivity in bulk crystals (Krause, Klimakow et al., 1990; Kauppinen et al.,
1997), while both cation vacancies and divacancies have been found in thin films (Liszkay et al., 1994; Keeble et al., 2011).

\section{Novel semiconductors: III-N, $\mathrm{SiC}$, and $\mathrm{ZnO}$}

The novel, often wide-band-gap, compound semiconductors are characterized by large size mismatch between the cation and anion. III-nitrides, $\mathrm{SiC}$, and $\mathrm{ZnO}$ are good examples of such semiconductors. While the traditional III-V and II-VI compounds tend to crystallize in a cubic (zinc blende) structure, these novel compounds prefer hexagonal symmetry (e.g., the wurtzite structure) and a smaller lattice constant. Another common aspect in these novel compounds is that anion ( $\mathrm{N}, \mathrm{O}$, and $\mathrm{C}$ ) vacancies have been blamed for various properties of the materials, while their direct observation is difficult or even impossible. Complex oxides suffer from the same syndrome. Unfortunately, due to the small size and natural positive charge of the anion vacancies, they are mostly elusive to positron annihilation spectroscopies as well. Cation vacancies, on the other hand, are readily observable, and in fact they have been shown to be responsible for many optoelectronic properties of these compounds. Positron annihilation spectroscopies have been widely applied to study III-nitrides, $\mathrm{SiC}$, and $\mathrm{ZnO}$-related materials.

$\mathrm{GaN}$ is by far the most studied of the III-nitrides. Early results of positron studies have been reviewed by Saarinen (2000) and bulk GaN crystal studies by Tuomisto (2010). Section IV.F discusses the identification and studies of $\mathrm{Ga}$ vacancies and their complexes in GaN, while Sec. IV.G covers the cation-anion vacancy clusters found in $\mathrm{InN}$ and InGaN alloys. A wide variety of studies of Ga-vacancy defects generated during epitaxial growth by MBE and metal-organic vapor phase deposition (MOCVD) have shown that similar effects related to growth temperature and stoichiometry can be found as in the traditional compounds, but in $\mathrm{GaN}$ oxygen impurities play a decisive role (Rummukainen et al., 2004; Hautakangas et al., 2006; Tuomisto, Paskova et al., 2007), and possibly also hydrogen (Hautakangas et al., 2006; Nykänen et al., 2012).

The hexagonal symmetry of the wurtzite lattice brings an additional property to the crystal compared to cubic lattices, namely, spontaneous polarization. Indeed the polarity of the growth surface strongly affects the Ga-vacancy defect formation and impurity incorporation in GaN (Rummukainen et al., 2004; Tuomisto, Saarinen, Lucznik et al., 2005; Tuomisto, Paskova et al., 2007). Some evidence of $\mathrm{N}$ vacancies with positrons has been found in irradiated (Tuomisto, Ranki et al., 2007) and Mg-doped GaN samples (Hautakangas et al., 2003), but further studies are clearly required. Thanks to the abundance of defects generated during growth of $\mathrm{GaN}$ or other III-nitrides, irradiation and ion implantation studies are rather scarce (Tuomisto, Pelli et al., 2007; Tuomisto, Ranki et al., 2007; Uedono et al., 2007; Moutanabbir et al., 2010; Mäki, Makkonen et al., 2011). Technologically important alloys such as $\mathrm{InGaN}$ and AlGaN have also been studied (Slotte et al., 2007; Chichibu et al., 2011; Uedono et al., 2012). The cation vacancies and their complexes found in these studies act as compensating centers in $n$-type material, as nonradiative recombination centers, and have sometimes been correlated with parasitic 
deep-level yellow luminescence in GaN (Saarinen et al., 1997).

$\mathrm{ZnO}$ is the II-VI counterpart of $\mathrm{GaN}$ and has been the object of a multitude of positron studies. Zn-vacancy defects have been identified in detail in both irradiated and as-grown material (Tuomisto et al., 2003; Tuomisto, Saarinen, Look, and Farlow, 2005), while some indirect evidence of $\mathrm{O}$ vacancies has also been found (Tuomisto, Saarinen, Look, and Farlow, 2005; Selim et al., 2007). The high resistance of ZnO's electrical and optical properties to deterioration under particle irradiation, the so-called radiation hardness, has generated significant interest in understanding the defect structure and behavior under thermal treatments. Indeed electron and ion irradiation experiments with subsequent thermal annealings (Tuomisto, Saarinen, Look, and Farlow, 2005; Chen et al., 2007; Chen, Betsuyaku, and Kawasuso, 2008; Zubiaga et al., 2008; Knutsen et al., 2012) have shown that the radiation hardness originates from the high mobility of $\mathrm{Zn}$ sublattice damage already at room temperature in $\mathrm{ZnO}$. The quest for $p$-type $\mathrm{ZnO}$ has led to many doping-by-implantation studies (Chen et al., 2004, 2005a, 2005b; Chen, Maekawa et al., 2005; Børseth et al., 2006, 2008; Neuvonen et al., 2009, 2011) where the $\mathrm{Zn}$ sublattice damage ( $\mathrm{Zn}$ vacancies) has been shown to strongly interact with the implanted impurities. Section IV.H discusses the role of $\mathrm{Li}$ in positron studies of $\mathrm{ZnO}$ - this abundant impurity in bulk crystals grown by the hydrothermal method has led to some scatter in positron data published over the years (Johansen, Zubiaga, Makkonen et al., 2011).

Silicon carbide ( $\mathrm{SiC}$ ) has the interesting property of existing in hundreds of crystalline forms, called polytypes, formed through different stackings of hexagonal planes. The simplest structures correspond to the zinc blende and wurtzite structures typical of III-V and II-VI compounds, called $3 \mathrm{C}$ and $2 \mathrm{H}$, respectively. The $3 C$ structure is the only cubic structure of $\mathrm{SiC}$. The $6 H \mathrm{SiC}$ polytype is the most studied $(6 H$ has triple periodicity in the stacking sequence of the hexagonal planes compared to $2 H$ ), with $4 H$ and $3 C$ gathering more and more interest. Silicon vacancies, silicon-carbon divacancies, and vacancy clusters have been studied in as-grown, irradiated, and implanted SiC (Brauer et al., 1996; Kawasuso et al., 1996; Polity, Huth, and Lausmann, 1999; Ling, Beling, and Fung, 2000; Henry et al., 2003; Aavikko et al., 2007). The optical ionization levels of vacancy defects have also been studied (Arpiainen et al., 2002) using positron spectroscopies. The detailed identification of the various vacancy defects has generated some discussion and even controversy (Rempel et al., 2002; Kuriplach et al., 2003). A probable reason for the relatively strong disagreement between some of the experimental and theoretical results is the crystal structure of $\mathrm{SiC}$ is that most of the experimental studies have been performed in $6 H \mathrm{SiC}$ (some in $4 H \mathrm{SiC}$ ), which has three (two) nonequivalent lattice sites on both sublattices. Hence the detailed balance between the defects on these lattice sites can affect the results quite strongly already for monovacancy defects (Wiktor et al., 2013), not to mention divacancies. For larger vacancy clusters the situation is the same as in other semiconductors, as the number of possible atomic configurations is already large for simpler structures.

\section{B. Vacancy-(multi)donor complexes in highly $n$-type doped silicon}

The decrease of the size of Si field-effect transistors requires extremely high doping densities in the drain and source regions. At donor concentrations above $10^{20} \mathrm{~cm}^{-3}$, however, the freeelectron concentration stops increasing with doping. This electrical deactivation was naturally attributed to the formation of compensating defects, while their identification has been much debated (Fahey, Griffin, and Plummer, 1989; Nylandsted Larsen et al., 1993; Packan, 1999). Many computational first-principles studies have addressed the electrical properties of these defects as well as their energetics including diffusion barriers [see, e.g., Pandey et al. (1988), Ramamoorthy and Pantelides (1996), Xie and Chen (1999), Christoph Mueller, Alonso, and Fichtner (2003), and Vollenweider, Sahli, and Fichtner (2010)]. Vacancy-impurity complexes were observed quite early in positron annihilation experiments (Lawther et al., 1995) but their exact structure remained unknown. Coincidence Doppler broadening experiments combined with theoretical calculations provided the optimal method for identification of these defects. In fact, in the original study where $V-\mathrm{As}_{n}$ complexes with $n=1-3$ were identified in Czochralski-grown highly As-doped Si (Saarinen et al., 1999), the coincidence experiments were performed with only one HPGe detector, with the gating signal provided by a $\mathrm{NaI}$ detector. This kind of coincidence experiment does not reduce the background as much as the coincidence experiment with two HPGe detectors (Alatalo et al., 1996; Asoka-Kumar et al., 1996; Szpala et al., 1996), nor does it narrow the energy resolution, but it provided information accurate enough in this case.

Figure 10 shows positron lifetime spectra in a float-zone(FZ-)grown undoped Si sample and in two 2-MeV electronirradiated samples: one undoped FZ grown and one As doped

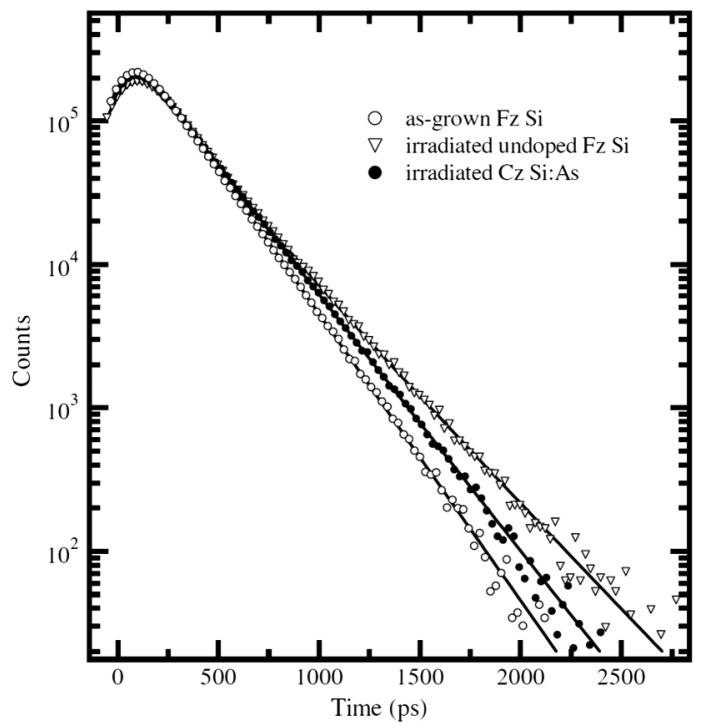

FIG. 10. Positron lifetime spectra in as-grown and in 2-MeV electron-irradiated Si samples. Positrons annihilate in the as-grown sample with a single lifetime of $220 \mathrm{ps}$ corresponding to delocalized positrons in the lattice. In the irradiated samples the experiments reveal vacancies with positron lifetimes of 250 ps ( $V$-As pair in $\mathrm{Cz}$ Si:As sample doped with $[\mathrm{As}]=1020 \mathrm{~cm}^{-3}$ ) and $300 \mathrm{ps}$ (divacancy in undoped FZ Si sample). From Saarinen and Ranki, 2003. 
$\left([\mathrm{As}]=10^{20} \mathrm{~cm}^{-3}\right)$ grown by the Czhochralski $(\mathrm{Cz})$ method. Positrons annihilate in the as-grown sample with a single lifetime of 220 ps corresponding to delocalized positrons in the lattice. In the irradiated samples, the experiments reveal vacancies with positron lifetimes of $250 \mathrm{ps}$ (Cz-grown Si:As) and 300 ps (undoped FZ-grown $\mathrm{Si}$ ), corresponding to monovacancy-sized defects and divacancies, respectively (Kauppinen et al., 1998; Saarinen et al., 1999). Isolated monovacancies are very mobile in $\mathrm{Si}$ already at room temperature (Watkins, 1986), and hence in undoped FZ-grown Si the vacancy defects that survive after irradiation are divacancies formed through the migration process, while in As-doped $\mathrm{Cz}$-grown $\mathrm{Si}$ the mobile monovacancies find As atoms and form stable vacancy-donor complexes (Lawther et al., 1995; Saarinen et al., 1999). Positron lifetime spectra in Cz-grown Si doped with As $\left(10^{19} \mathrm{~cm}^{-3}\right)$ or $\mathrm{P}\left(10^{20} \mathrm{~cm}^{-3}\right)$ also have a single component of about 220 ps corresponding the bulk lifetime $\tau_{B}$ in Si. Hence, these materials are free of vacancies trapping positrons. The average positron lifetime is clearly higher in as-grown $\mathrm{Si}$ doped with As to a higher level $\left([\mathrm{As}]=10^{20} \mathrm{~cm}^{-3}\right)$, giving $\tau_{\mathrm{av}}=232 \mathrm{ps}$ at room temperature. Furthermore, the lifetime spectrum has two components, the longer of which is $\tau_{2}=250 \pm 3$ ps. Both $\tau_{\text {av }}$ and $\tau_{2}$ are almost constant as a function of temperature. The twocomponential lifetime spectrum and the increase of $\tau_{\text {ave }}$ above the bulk lifetime $\tau_{B}$ are clear signs that native vacancies exist in $\operatorname{Si}\left([\mathrm{As}]=10^{20} \mathrm{~cm}^{-3}\right)$. The second lifetime component $\tau_{2}=250 \pm 3$ ps is characteristic of the positron annihilations at a monovacancy defect.

In 2-MeV electron-irradiated samples the average positron lifetime is longer than in as-grown samples, indicating that irradiation-induced vacancies are observed (Fig. 10). In $\operatorname{Si}\left([\mathrm{P}]=10^{20} \mathrm{~cm}^{-3}\right)$ irradiated to the fluence $5 \times 10^{17} \mathrm{~cm}^{-2}$ the lifetime spectrum can be decomposed and the vacancy component $\tau_{2}=250 \pm 3 \mathrm{ps}$ is obtained (Saarinen et al., 1999). Irradiated $\mathrm{Si}\left([\mathrm{As}]=10^{20} \mathrm{~cm}^{-3}\right)$ exhibits only a single positron lifetime of about $247 \pm 2$ ps, almost independently of the irradiation fluence. This behavior can be explained by a total positron trapping at irradiation-induced vacancy defects. When the vacancy concentration exceeds $10^{18} \mathrm{~cm}^{-3}$, all positrons annihilate at the irradiation-induced vacancy defects with the lifetime $247 \pm 2$ ps and no annihilations take place at the delocalized bulk states or at the native vacancies detected before irradiation. The vacancy concentration of $\geq 10^{18} \mathrm{~cm}^{-3}$ is consistent with the expected introduction rate in electron-irradiated heavily $n$-type Si. The same positron lifetime characteristic of a monovacancy, $\tau_{V}=248 \pm 3 \mathrm{ps}$, is in fact observed for three different types of samples: (i) as-grown $\mathrm{Si}([\mathrm{As}]=$ $\left.10^{20} \mathrm{~cm}^{-3}\right)$, (ii) electron-irradiated $\operatorname{Si}\left([\mathrm{As}]=10^{20} \mathrm{~cm}^{-3}\right)$, and (iii) electron-irradiated $\operatorname{Si}\left([\mathrm{P}]=10^{20} \mathrm{~cm}^{-3}\right.$ ) (Saarinen et al., 1999).

Doppler broadening experiments using the two-detector coincidence technique have been used to identify these monovacancy defects in more detail. In the samples containing vacancy defects, the Doppler broadening represents the superimposed distribution $\rho(p)=(1-\eta) \rho_{B}(p)+\eta_{V} \rho_{V}(p)$, where $\rho_{B}(p)$ and $\rho_{V}(p)$ are the momentum distributions in the lattice and at the vacancy, respectively. The lifetime results (Fig. 10) can be used to determine the fraction of positrons annihilating at vacancies $\eta_{V}=\left(\tau_{\text {ave }}-\tau_{B}\right) /\left(\tau_{V}-\tau_{B}\right)$

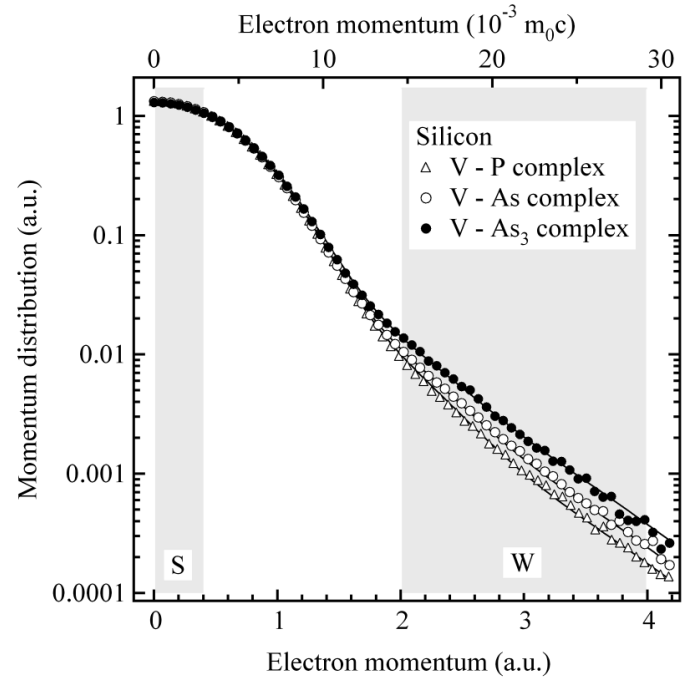

FIG. 11. Experimental (markers) and calculated (solid curves) coincidence Doppler broadening spectra in vacancy-donor complexes in $\mathrm{Si}$, showing the perfect match between theory and experiment. Adapted from Saarinen and Ranki, 2003.

[see Eqs. (26) and (27)]. Since the momentum distribution in the lattice $\rho_{B}(p)$ can be measured in the reference sample, the distributions $\rho_{V}(p)$ at vacancies can be decomposed from the measured spectrum $\rho(p)$. They are shown in Fig. 11 for the monovacancies observed in as-grown $\mathrm{Si}([\mathrm{As}]=$ $\left.10^{20} \mathrm{~cm}^{-3}\right)$ as well as in irradiated $\operatorname{Si}\left([\mathrm{As}]=10^{20} \mathrm{~cm}^{-3}\right)$ and $\operatorname{Si}\left([\mathrm{P}]=10^{20} \mathrm{~cm}^{-3}\right)$.

The momentum distributions at vacancies $\rho_{V}(p)$ indicate large differences in the higher momenta ( $p>2$ a.u.), where the annihilation with core electrons is the most important contribution (Fig. 11). Since the core electron momentum distribution is a specific characteristic of a given atom, the differences between the spectra in Fig. 11 indicate different atomic environments of the vacancy in each of the three cases. Because in both $\mathrm{Si}(Z=14)$ and $\mathrm{P}(Z=15)$ the $2 p$ electrons constitute the outermost core electron shell, the core electron momentum distributions of these elements are very similar. The crucial difference in the core electron structures of $\mathrm{Si}, \mathrm{P}$, and As is the presence of ten $3 d$ electrons in As. The overlap of positrons with the As $3 d$ electrons is much stronger than with the more localized $\mathrm{Si}$ or $\mathrm{P} 2 p$ electrons. The large intensity of the core electron momentum distribution is thus a clear sign of As atoms surrounding the vacancy. The $2-\mathrm{MeV}$ electron irradiation creates vacancies and interstitials as primary defects, both of which are mobile at room temperature (Watkins, 1986).

In heavily $n$-type $\mathrm{Si}$ the donor atom may capture the vacancy and form a vacancy-impurity pair (Watkins, 1986). The monovacancy detected in heavily $\mathrm{P}$-doped $\mathrm{Si}$ is thus the $V$-P pair. Similarly, it is natural to associate the electronirradiation-induced vacancy in $\operatorname{Si}\left([\mathrm{As}]=10^{20} \mathrm{~cm}^{-3}\right)$ with a $V$-As pair. An even stronger signal from As is seen in the asgrown $\operatorname{Si}\left([\mathrm{As}]=10^{20} \mathrm{~cm}^{-3}\right)$. A linear extrapolation of the intensity of the distribution suggests that the native complex is $V$-As $s_{3}$, i.e., the vacancy is surrounded by three As atoms. These identifications are confirmed by theoretical results that are in very good agreement at both low and high momenta (Fig. 11). The theoretical calculations strongly support the 
experimental defect identifications that (i) vacancies complexed with single donor impurities are detected in electronirradiated $\mathrm{P}$ and As-doped $\mathrm{Si}$, and (ii) the native defect in $\mathrm{Si}\left([\mathrm{As}]=10^{20} \mathrm{~cm}^{-3}\right)$ is a vacancy surrounded by three As atoms.

The existence of $V-\mathrm{As}_{3}$ complexes in heavily As-doped $\mathrm{Cz}$-grown $\mathrm{Si}$ is consistent with the defect formation and diffusion mechanisms described theoretically (Ramamoorthy and Pantelides, 1996). The calculated formation energies of $V-\mathrm{As}_{n}(n>2)$ complexes are negative, suggesting that total deactivation of As takes place at any doping level (Pandey et al., 1988; Ramamoorthy and Pantelides, 1996). The $n$-type conductivity of $\mathrm{Si}(\mathrm{As})$ is possible only because the creation of defect complexes is limited by kinetic processes such as the migration of defects. At high temperature the diffusion of As starts by the formation of $V$-As pairs, which can migrate to form $\mathrm{V}$-As $\mathrm{A}_{2}$ complexes (Mathiot and Pfister, 1983). The calculations (Ramamoorthy and Pantelides, 1996) predict that these complexes can diffuse until they stop at the substitutional As forming the $V-\mathrm{As}_{3}$ complex. In addition, no $V-\mathrm{As}_{3}$ are found at the lower doping level of $[\mathrm{As}]=10^{19} \mathrm{~cm}^{-3}$ (Saarinen et al., 1999), most likely because the average distance between the donor atoms is too large and the migrating $V$-As and $V$-As 2 may dissociate before creating larger complexes. In fact, similar observations and conclusions can be made in heavily P-doped and Sb-doped Si as well (Ranki, Pelli, and Saarinen, 2004; Rummukainen et al., 2005). The formation mechanism of the vacancy-donor complexes $(V-D)$ has been studied in annealing experiments of the electron-irradiated samples.

A general scheme for vacancy-donor complex formation can be deduced from positron experiments (Ranki, Pelli, and Saarinen, 2004). The electron irradiation creates vacancydonor pairs $\left(V-D_{1}\right)$ that start to migrate at $400-450 \mathrm{~K}$. The migrating and dissociating $V-D_{1}$ defects form more stable $V-D_{2}$ defects and some divacancies. The divacancies start to anneal away soon after they are formed, around 450-500 K, and at $600 \mathrm{~K}$ there are only $V-D_{2}$ defects left. In the case of As and $\mathrm{P}$ doping, the $V-D_{2}$ defects start to migrate at 650$700 \mathrm{~K}$ forming $V-D_{3}$ defects. The formation of $V-D_{2}$ and $V-D_{3}$ depends heavily on the doping concentration. The vacancy-impurity complex formation can be explained by the ring diffusion mechanism, where the opposite charges of the vacancy and the donor atom bind the migrating complex together (Ramamoorthy and Pantelides, 1996; Pankratov et al., 1997; Xie and Chen, 1999; Ranki, Nissilä, and Saarinen, 2002). These observations are consistent with results obtained by EPR and deep-level transient spectroscopy (Nylandsted Larsen, Christensen, and Petersen, 1999). The estimated activation energies are also in very good agreement with theoretical calculations of migration barriers (Xie and Chen, 1999; Vollenweider, Sahli, and Fichtner, 2010).

\section{The vacancy-fluorine complex in silicon and silicon-germanium alloys}

The effect of fluorine on the behavior of vacancies $(V)$ and interstitials $(I)$ in Si has been of great interest (El Mubarek and Ashburn, 2003). It has been incorporated in Si in several device processes through ion implantation (Ma, 1992;
Williams and Ashburn, 1992; Downey, Osburn, and Marcus, 1997). Fluorine in silicon exhibits the ability to retard the diffusion of boron, either when coimplanted as $\mathrm{BF}_{2}$, or as separate implants. This allows a strategy for ultrashallow junction technologies. The key is the formation of fluorine-vacancy (F-V) and fluorine-interstitial (F-I) complexes. The incomplete picture of the basic behavior of $\mathrm{F}$ in $\mathrm{Si}$ has stood in the way of the realization of its full potential. Positron annihilation spectroscopy has been used to study the effects of $\mathrm{F}$ on the vacancies in implantation processing of $\mathrm{Si}$ and Si-Ge alloys. These studies provide an illustrative example of the study of ion implantation damage and subsequent identification of the defects created by processing.

Figure 12 shows the $S$ parameter as a function of positron implantation energy for a selection of F-implanted $\left(E_{\text {ion }}=\right.$ $0.5 \mathrm{MeV}$ ) and subsequently annealed $\mathrm{Cz}$-grown $\mathrm{Si}$ samples (Pi, Burrows, and Coleman, 2003). The trapping of positrons at vacancy defects leads to a higher $S$ parameter compared with that for the defect-free lattice. The $S$ values presented in Fig. 12 have been normalized with respect to that for a bulk virgin Si sample for which $S$ is thus 1 . The $0.5 \mathrm{MeV}$ implantation energy of $\mathrm{F}$ ions corresponds to a projected range $R_{p}=$ $0.9 \mu \mathrm{m}$. The vacancy distribution extends to around $2.3 \mu \mathrm{m}$. This abnormally deep distribution of vacancies is probably due to $\mathrm{F}$ atoms strongly reacting with interstitials during ion implantation and allowing the survival of most of the vacancies. The dip that appears around $11 \mathrm{keV}$ (Fig. 12) in the $S$ curve for the as-implanted sample and deepens strongly in the annealings corresponds to the region close to $R_{p}$. It is known from earlier studies that $\mathrm{F}$ and $\mathrm{O}$ reduce $S$ when they are associated with vacancies because of the large momenta of

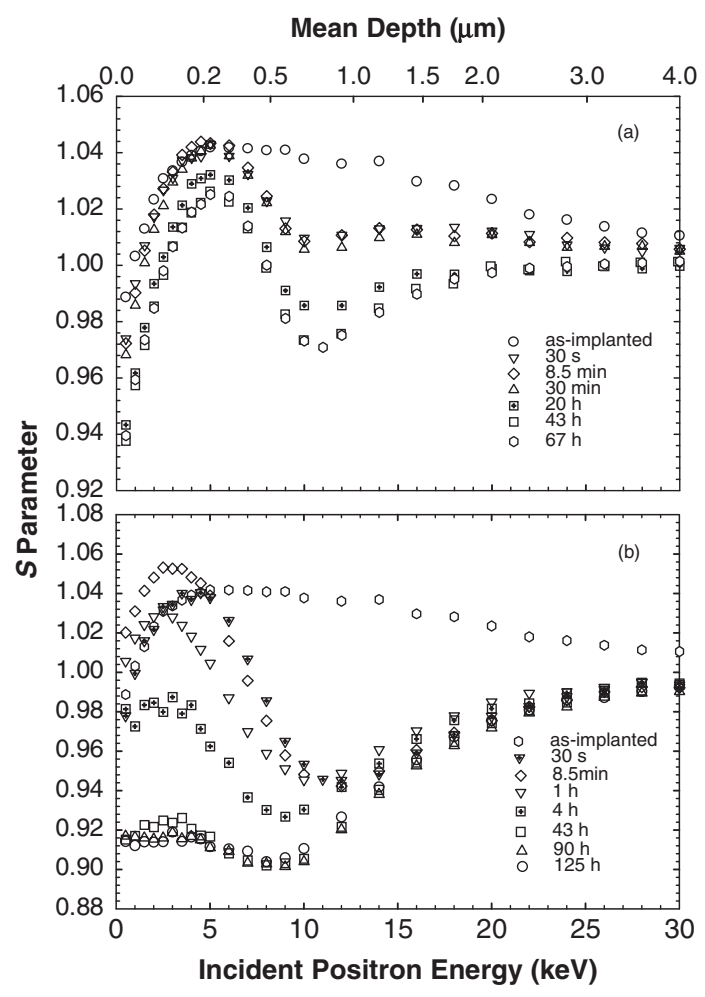

FIG. 12. $S$ parameter depth profiles for F-implanted Si samples annealed at (a) $400{ }^{\circ} \mathrm{C}$ for up to $67 \mathrm{~h}$ and (b) $700^{\circ} \mathrm{C}$ for up to $125 \mathrm{~h}$. From Pi, Burrows, and Coleman, 2003. 


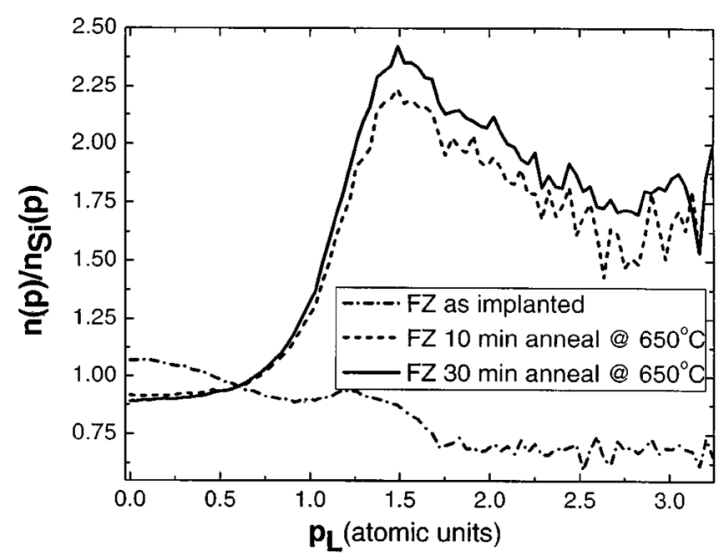

FIG. 13. Coincidence Doppler broadening spectra, normalized to the spectrum for silicon, showing relative intensity vs electron momentum. Peaks in the data from annealed samples match the "fingerprint" of fluorine. From Simpson et al., 2004.

their outermost-shell electrons (Coleman, Chilton, and Baker, 1990; Fujinami, 1996; Uedono et al., 1997). Hence based on these depth profiles it seems evident that $\mathrm{F}$ decorates some vacancies around $R_{p}$ already right after implantation, and this effect becomes stronger after the annealings.

Figure 13 shows coincidence Dopper broadening data (ratio curves) from similarly implanted FZ-grown Si samples. The ratio curve for the as-implanted sample is typical of undecorated vacancy defects (or in the case of silicon, the divacancy) with the low-momentum region having intensity above 1 and the high-momentum region below 1 . The data obtained in the F-implanted samples after annealing show a very strong effect at high momenta (ratios up to 2.5 at around 1.5 a.u.), while the $S$ parameter region at low momenta goes below 1. This behavior is typical of impurities with outershell electrons with large momenta (such as $\mathrm{O}$ or $\mathrm{F}$ ) or with large number of outer-shell electrons (e.g., As, see previous section). The concentrations of $3 d$ impurities are very low in FZ-grown $\mathrm{Si}$, as is the concentration of $\mathrm{O}$, and hence it is natural to assign the strong peak at 1.5 a.u. as the $F$ fingerprint. Figure 14 shows similar data for F-implanted Si-Ge

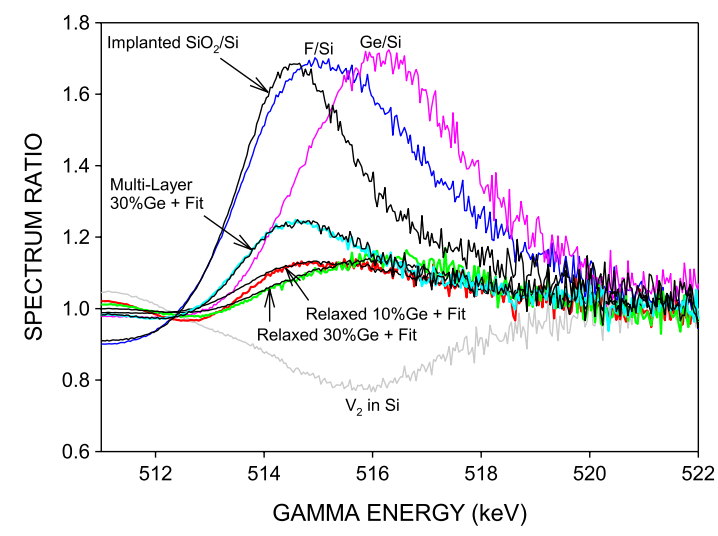

FIG. 14 (color online). Ratios of the annealed samples of relaxed $10 \%$ and $30 \% \mathrm{Ge}$ and multilayer $30 \% \mathrm{Ge}$ at $\sim 2 \mathrm{keV}$. Best fits are shown on top of data. Ratios of implanted $\mathrm{SiO}_{2} / \mathrm{Si}, \mathrm{F} / \mathrm{Si}, \mathrm{Ge} / \mathrm{Si}$, and $V_{2}$ in $\mathrm{Si} / \mathrm{Si}$ are shown for reference. All spectra are divided by a Si spectrum. From Edwardson et al., 2012. alloys. The figure shows that the effects of $\mathrm{Ge}, \mathrm{O}$, and $\mathrm{F}$ in the silicon lattice, although qualitatively quite similar, can be separated. The differences in the positions of the signatures (511 keV corresponds to 0 a.u., and $522 \mathrm{keV}$ to 6 a.u.) between Figs. 13 and 14 originate from different detector resolutions: the data in Fig. 13 have been measured with a two-HPGe-detector coincidence setup that narrows the resolution by a factor of $\sim 1.4$ compared to the single-detector measurement that was used for acquiring the data in Fig. 14. The fast convergence to a ratio of $\sim 1$ above $\sim 516 \mathrm{keV}$ (2.7 a.u.) in Fig. 14, not observed in Fig. 13, is due to the dominance of the high background in the single-detector measurement compared to the two-HPGe-detector coincidence measurement.

The fluorine-vacancy complexes have indeed been predicted as the cause for the reduction of diffusion of boron (Diebel and Dunham, 2004; Lopez et al., 2005; Lopez and Fiorentini, 2006; Vollenweider, Sahli, and Fichtner, 2009), and they have been experimentally found also by EPR (Umeda et al., 2010). TEM has been used to follow the generation of larger F precipitates in solid-phase epitaxy of Si (Boninelli et al., 2006, 2008), consistent with the predicted vacancy-fluorine complex dynamics. The boron-diffusionreducing properties of fluorine and the strong vacancyfluorine interactions extend from $\mathrm{Si}$ also to $\mathrm{Si}-\mathrm{Ge}$ alloys (El Mubarek et al., 2005) and Ge (Jung et al., 2012). The positron annihilation experiments have given a significant contribution to the understanding of the phenomena related to fluorine implantation and its effects on vacancy passivation and suppression of boron transient enhanced diffusion.

\section{The EL2 defect in gallium arsenide}

One of the most prominent examples of the application of positron annihilation spectroscopy in identification of technologically important defects is the investigation of the socalled EL2 midgap donor defect in GaAs. The importance of this defect stemmed from its central role in the growth of undoped semi-insulating (SI) GaAs (Martin and MakramEbeid, 1986; Kaminska and Weber, 1993). A key property of EL2 is its optically induced metastability: it can be permanently converted to the neutral metastable state EL2* under $0.8-1.5 \mathrm{eV}$ illumination at temperatures below $100 \mathrm{~K}$. The photoquenching occurs without generation of any new electrical or optical signals that could be associated with the metastable state EL2*. Identification of the atomic structure of this defect was the focus of a considerable effort in the late 1980s and early 1990s. Another metastable defect whose identification proved to be a challenging task (around the same time) was the so-called DX center (Mooney, 1990; Mäkinen et al., 1993) in $\mathrm{Al}_{1-x} \mathrm{Ga}_{x} \mathrm{As}$ that produces persistent photoconductivity.

Figure 15 shows results of positron experiments where SI GaAs crystals with two different EL2 concentrations were cooled in darkness and illuminated in situ with $1.2 \mathrm{eV}$ light (Krause, Saarinen et al., 1990). To check that the EL2 defects were photoquenched by the illumination, infrared absorption was also measured. It is clearly seen that after the photoquenching of the EL2 centers at low temperatures (below $100 \mathrm{~K}$ ) there is a clear increase of the positron annihilation 


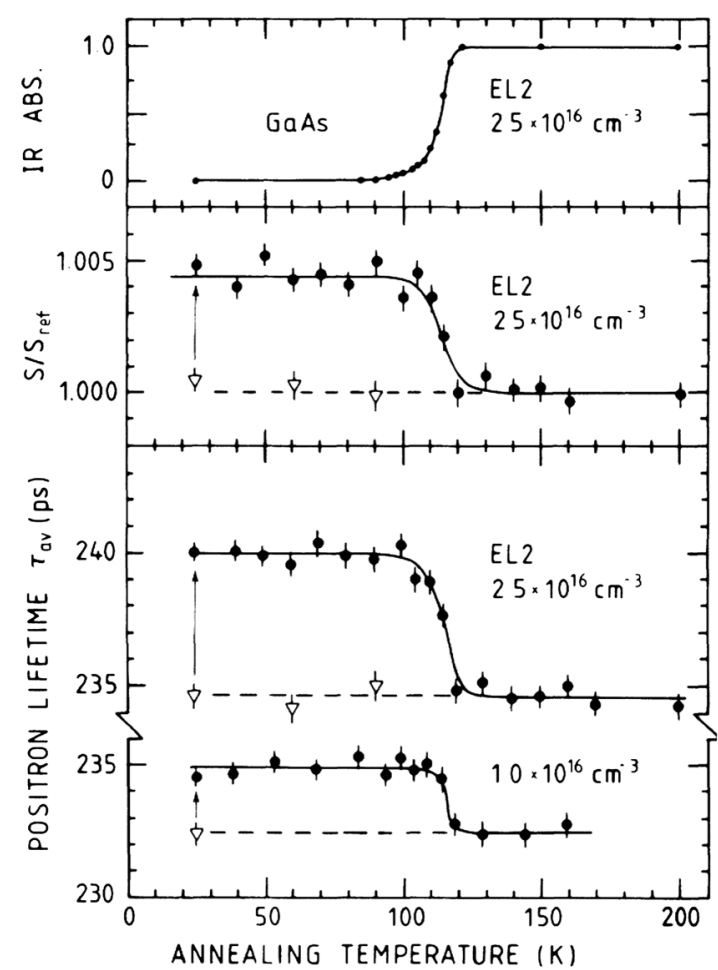

FIG. 15. The average positron lifetime $\tau_{\mathrm{av}}$ and annihilation lineshape parameter $S$ in semi-insulating GaAs as functions of isochronal annealing temperature after $1.2-\mathrm{eV}$ illumination at $25 \mathrm{~K}$. The illumination transforms EL2 into the metastable state and corresponding changes in positron parameters are indicated by arrows. The open triangles with dashed lines below $100 \mathrm{~K}$ represent the reference levels where EL2 is in the stable state. The normalized infrared absorption coefficient is shown in the top panel. All measurements have been made in darkness at $25 \mathrm{~K}$. From Krause, Saarinen et al., 1990.

parameters $\tau_{\text {av }}$ and $S$. This property is reproducible and has been since observed in various SI GaAs crystals, indicating that vacancy defects are generated by the photoquenching of EL2. In addition, the concentration of these vacancy defects is correlated with the total EL2 concentrations (Le Berre et al., 1994; Saarinen et al., 1994). Further, both the time constant and photon energy dependence of the effect show that the vacancy generation is due to the EL2 conversion to the metastable state (Saarinen et al., 1994). As an example, Fig. 16 shows the optical cross section for the creation of the metastable state of the EL2 defect and the metastable vacancy. A similar approach can be used to study the photoionization levels of vacancy defects (Kuisma et al., 1996).

The identification of the metastable vacancy defect was possible by comparison of the second lifetime component extracted from the lifetime spectra after illumination, which in SI GaAs was found to be $\tau_{2}=247 \pm 3$ ps. In this particular state of the sample, two kinds of vacancies may trap positrons: the $\mathrm{Ga}$ vacancies often found as native defects in SI GaAs and the metastable vacancies observed after illumination. It should be noted that in Fig. 15 the average positron lifetime in dark is a bit longer than the bulk lifetime $\tau_{B}=$ $230 \mathrm{ps}$ of GaAs. Hence the second lifetime $\tau_{2}$ should be a superposition of the lifetimes at Ga vacancies $\tau_{V}=260 \mathrm{ps}$ and at the metastable vacancies with the positron lifetime $\tau_{V^{*}}$.

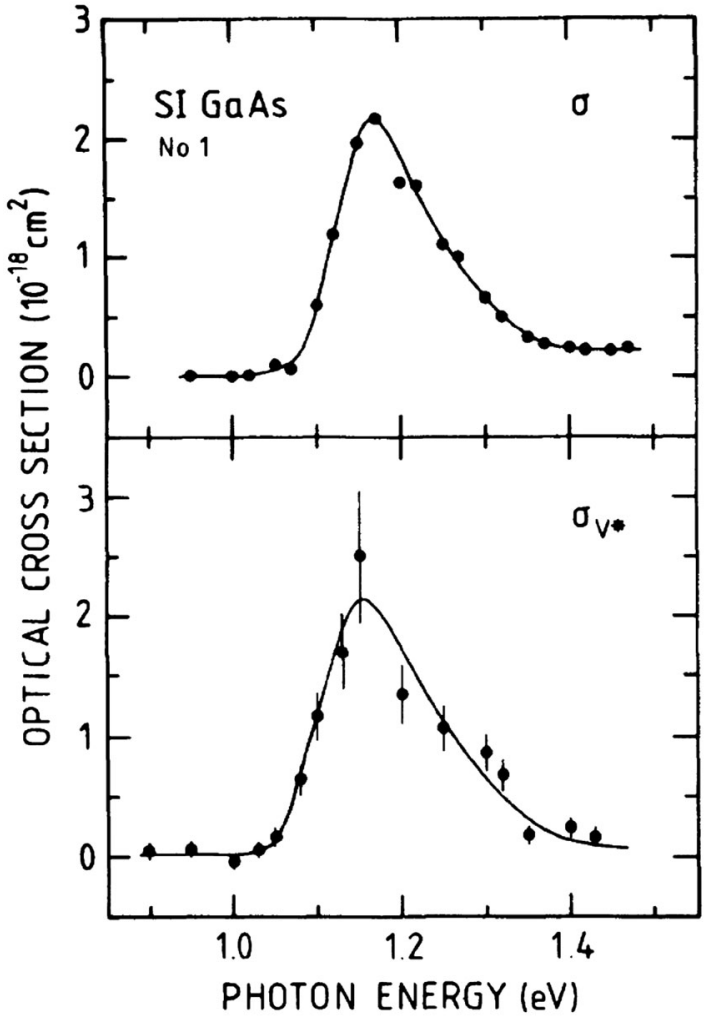

FIG. 16. Optical cross section for the creation of the metastable vacancy as a function of the photon energy in a SI GaAs sample. The data in the upper part are obtained from IR absorption measurements and in the lower part from positron lifetime measurements. Measurement temperature was $25 \mathrm{~K}$. From Saarinen et al., 1994.

Taking into account the positron trapping at native Ga vacancies, one could estimate that the positron lifetime at the metastable vacancy is $\tau_{V^{*}} \sim 245$ ps (Saarinen et al., 1994). This lifetime is clearly shorter than the values at the Ga vacancies and As vacancies (260-300 ps). Hence the metastable structure of the EL2 defect has an associated open volume that is smaller than that of a monovacancy in GaAs. These observations gave strong support to the model where the EL2 defect consists of an isolated As antisite defect that relaxes toward the interstitial position in the metastable state. The reaction $\mathrm{As}_{\mathrm{Ga}} \rightarrow V_{\mathrm{Ga}}+\mathrm{As}_{i}$ creates the $V_{\mathrm{Ga}}-\mathrm{As}_{i}$ pair that is the metastable vacancy $V^{*}$ with smaller open volume than that of the isolated Ga vacancy (Chadi and Chang, 1988; Dabrowski and Scheffler, 1988). There is no evidence of positron trapping at the stable state of EL2, consistent with the idea that open volume is not present then. Theoretical calculations of the positron states (Laasonen et al., 1991) also show that the proposed atomic configuration of the metastable EL2 indeed localizes a positron, with a specific lifetime in between the bulk and Ga-vacancy lifetimes.

The work on the EL2 defect also demonstrates the capability of positron annihilation methods to identify transition levels in the band gap of a semiconductor. In the above case, the optically induced transition involves a strong lattice relaxation that makes the defect appear in positron annihilation experiments. Another possibility is the change in the charge state of the vacancy defect, affecting the sensitivity of positrons (i.e., the positron trapping coefficient and its 
temperature behavior) to the defect in question. The effects of vacancy charge states are discussed in more detail in the next sections. Good examples of observation of photoionization of defect levels include the work on vacancies in $\mathrm{SiC}$ (Arpiainen et al., 2002) and vacancy clusters in diamond (Dannefaer, $\mathrm{Pu}$, and Kerr, 2001; Mäki et al., 2009).

\section{E. The gallium vacancy-tellurium complex in gallium arsenide}

The studies of Te-doped GaAs provide an instructive example of how temperature-dependent positron lifetime measurements show the competition of negatively charged vacancies and negative ions in positron trapping at low temperatures, and how the changes in their relative concentrations affect the data. In addition, coincidence Doppler broadening studies of $n$-type GaAs show how to distinguish between the two possible sublattices of the observed vacancies, as positron lifetime experiments alone are not enough for this task: the same lifetime can be produced by a Ga vacancy, an As vacancy, and a vacancy-dopant complex when all atoms are on lattice sites. Te is incorporated in the As sublattice only (Hurle, 1979).

Figure 17 from Gebauer et al. (1999) shows positron lifetime data measured in Te-doped Cz-grown GaAs samples with varying carrier concentration $(n=5 \times$ $10^{16}-10^{18} \mathrm{~cm}^{-3}$ ). The $\mathrm{Zn}$-doped GaAs reference does not show any trapping of positrons at vacancies. The slight increase of the average positron lifetime with measurement temperature comes from the thermal expansion of the lattice (Le Berre et al., 1995). The average positron lifetime $\tau_{\text {av }}$ in the Te-doped samples is above $\tau_{B}=229-230 \mathrm{ps}$ found in GaAs: $\mathrm{Zn}$, indicating positron trapping at vacancies. The temperature dependence of $\tau_{\mathrm{av}}$ in GaAs:Te is typical when

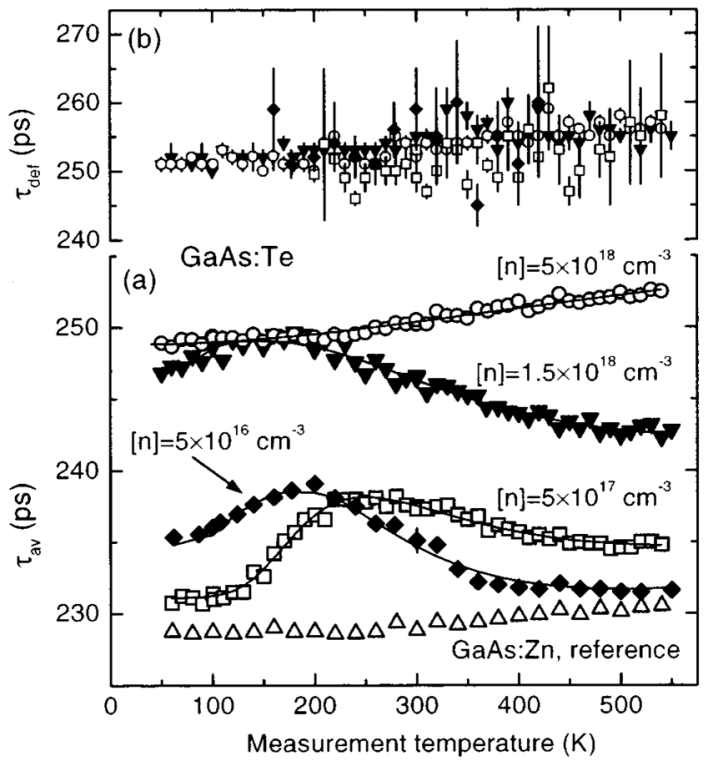

FIG. 17. (a) Average positron lifetime $\tau_{\text {ave }}$ and (b) defect-related lifetime $\tau_{\text {def }}$ vs measurement temperature in Te-doped GaAs compared to a GaAs: $\mathrm{Zn}$ reference. Spectral decomposition was not reliable for $T>350 \mathrm{~K}$ for the $5 \times 10^{16} \mathrm{~cm}^{-3}$ doped sample $\left(\tau_{\mathrm{av}}\right.$ is close to $\left.\tau_{b}\right)$. Lines are fits according to the temperaturedependent trapping model. From Gebauer et al., 1999. positrons are trapped at negatively charged vacancies and negative ions. The negative ions with a lifetime close to $\tau_{B}$ trap positrons in their shallow potential only at low temperature. Negative ions can be attributed to intrinsic defects, such as $\mathrm{Ga}_{\mathrm{As}}$, or extrinsic impurities, but positron annihilation alone does not allow their detailed identification. With increasing temperature positrons escape from the ions and a larger fraction annihilates at vacancies, causing the increase of $\tau_{\mathrm{av}}$ between 100 and $200 \mathrm{~K}$. The decrease of $\tau_{\mathrm{av}}$ at $T>200 \mathrm{~K}$ in the medium-doped samples indicates positron trapping at negative vacancies.

The data are fitted with a model taking into account positron trapping and detrapping at the shallow Rydberg states around negative ions and vacancies as well as the $T^{-1 / 2}$ dependence for positron trapping at negatively charged defects. The temperature dependence of positron trapping is discussed in Sec. II, while a detailed discussion can be found in, e.g., Krause-Rehberg and Leipner (1999). The parameters describing the temperature dependence of positron trapping are similar in all samples, the binding energy of positrons to the Rydberg states was $E_{\text {ion }}=65 \pm 20 \mathrm{meV}$, and only the concentrations of the ions and vacancies relative to each other change (Gebauer et al., 1999). Positron trapping at vacancies is practically saturated in the most highly doped sample and hence $\tau_{\text {ave }}$ reflects the slight decrease of the defect-related lifetime $\tau_{D}$ with temperature. This might be attributed to lattice expansion too, although the effect is larger than in the reference. It is important that $\tau_{D}=254 \pm 3$ ps at $300 \mathrm{~K}$ is the same in all samples and exhibits the same temperature dependence, suggesting that the vacancies are similar in all samples. This suggestion is confirmed by the observation that the Doppler broadening parameters $(S$ and $W$ ) change linearly with the average positron lifetime in these samples (Gebauer et al., 1999).

Figure 18 from Gebauer et al. (1999) shows the highmomentum part of the annihilation momentum distribution ratio curve for the vacancies in GaAs:Te compared to those found in GaAs:Si. The core annihilation is more intense in GaAs:Te than in GaAs:Si in the momentum range $p_{L}=$ $(10-20) \times 10^{-3} m_{0} c(1.3-2.6$ a.u.). The observations of the momentum distribution can be explained as follows. In bulk GaAs, the dominating contribution to the core annihilation comes from Ga $3 d$ electrons $(Z=31)$ (Alatalo et al., 1996). The As $3 d$ electrons $(Z=33)$ are more tightly bound and hence the momentum distribution is broader and the intensity of the core annihilation is reduced. Positron annihilation at the $\mathrm{Si}_{\mathrm{Ga}}-V_{\mathrm{Ga}}$ complexes in GaAs:Si occurs mainly with $3 d$ electrons from As. Thus, the momentum distribution should be broader compared to the bulk. This is, in fact, observed. In contrast, at As vacancies the momentum distribution should be narrower and more intense because annihilation occurs mainly with the Ga $3 d$ electrons.

In tellurium the main contribution to the core annihilation comes from $4 d$ electrons that are less strongly bound than the As $3 d$ electrons in GaAs. They contribute therefore to the core annihilation more at lower momenta and have a steeper momentum distribution. The theoretical calculations shown in Fig. 18(b) demonstrate this effect very well. A similar difference has been noted by comparing results from bulk InP, GaSb, and GaAs (Alatalo et al., 1995) and for 


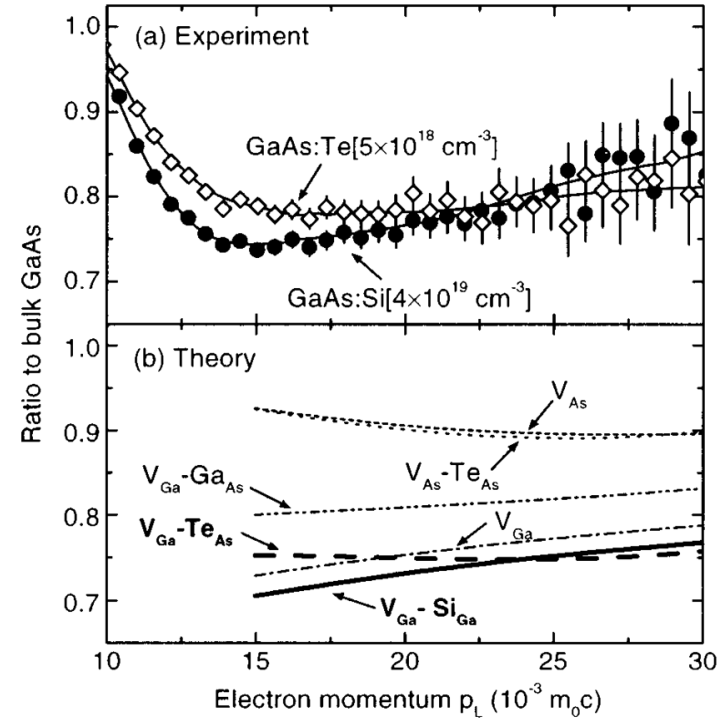

FIG. 18. (a) High-momentum part of the positron annihilation momentum distribution (normalized by taking the ratio to a GaAs: $\mathrm{Zn}$ reference) for the vacancies in GaAs:Te and GaAs:Si. The spectra (total area $3.5 \times 10^{7}$ counts) were brought to unity and scaled to full trapping at the vacancies before normalization. Lines result from smoothing and serve to guide the eye only. (b) Ratio of the momentum density to bulk GaAs for different vacancies in $\mathrm{GaAs}$ from theoretical calculations. The curves for $V_{\mathrm{Ga}}-\mathrm{Te}_{\mathrm{As}}$ and $V_{\mathrm{As}}-\mathrm{Si}_{\mathrm{Ga}}$ complexes are highlighted to emphasize the good agreement with the respective experimental data in GaAs:Te and GaAs: $\mathrm{Si}$. The theoretical curves are not accurate for $p_{L}<15 \times 10^{-3} m_{0} c$ and hence are omitted. From Gebauer et al., 1999.

Zn-impurity-P-vacancy complexes in InP (Alatalo et al., 1996). The shape of the momentum distribution measured in GaAs:Te therefore indicates that the vacancies are neighbored by $\mathrm{Te}$ atoms. Because $\mathrm{Te}$ resides on the As sublattice, the vacancy must be on the Ga sublattice. Hence the vacancies in GaAs:Te can be identified as Ga-vacancy$\mathrm{Te}_{\mathrm{As}}$ complexes. This assignment of the reduced positron lifetime at the $\mathrm{Ga}$ vacancies in GaAs:Te (254 ps) compared to the Ga vacancies in GaAs: $\mathrm{Si}$ (262 ps) is natural, as the large Te atom can be expected to decrease the open volume of the neighboring Ga vacancy.

\section{F. The gallium vacancy and its complexes in gallium nitride}

Positron studies of GaN and the III-nitrides started in the second half of the 1990s, with five papers published by different groups in 1997 (Cho et al., 1997; Dannefaer, Puff, and Kerr, 1997; Jorgensen et al., 1997; Saarinen et al., 1997; Suzuki et al., 1997). These reports give an instructive example of how a new material is studied and how the results can be interpreted just by using knowledge acquired in previous studies of another material. GaAs was a natural material of comparison, as it was the compound semiconductor that had been studied extensively with positron annihilation spectroscopy. The identification of $\mathrm{Ga}$ vacancies by Saarinen et al. (1997) was possible thanks to the growth of high-nitrogen-pressure bulk GaN single crystals of sufficient size for experimentation.

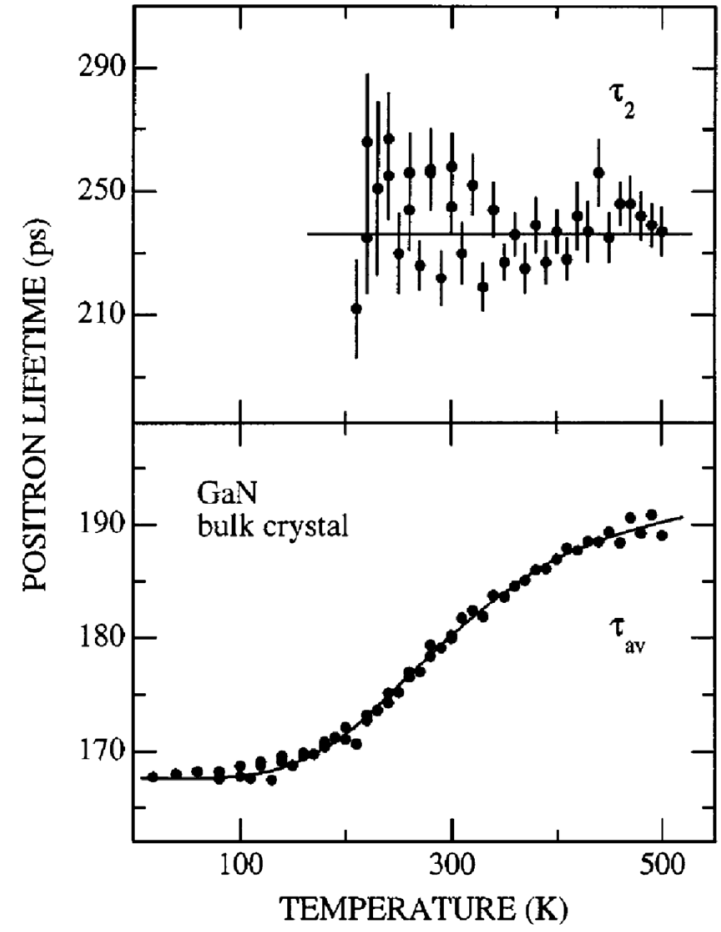

FIG. 19. The average positron lifetime $\tau_{\mathrm{av}}$ and the lifetime component $\tau_{2}$ vs measurement temperature for GaN bulk crystal. The lifetime component $\tau_{2}$ could be decomposed only at $T>200 \mathrm{~K}$. The solid lines are drawn to guide the eye. From Saarinen et al., 1997.

Figure 19 shows the positron lifetime data obtained as a function of temperature in such crystals. The average positron lifetime in the GaN bulk crystal is constant $\tau_{\mathrm{av}}=167 \mathrm{ps}\left(\tau_{\mathrm{av}}\right.$ in the figure) at temperatures $T=10-150 \mathrm{~K}$ but increases up to $\tau_{\mathrm{av}}=191 \mathrm{ps}$ at $500 \mathrm{~K}$. The lifetime spectra recorded at 200-500 K can be decomposed into two components. The longer-lifetime component is constant, $\tau_{2}=235 \pm 5 \mathrm{ps}$ (Fig. 19), as a function of temperature. By also fixing this lifetime, the spectra measured at $10-200 \mathrm{~K}$ could be decomposed. The lifetime component $\tau_{1}$ is a constant, $\tau_{1}=164 \pm$ $1 \mathrm{ps}$, at $10-150 \mathrm{~K}$ and then decreases to about $\tau_{1}=140 \mathrm{ps}$ at $500 \mathrm{~K}$. The two-component lifetime spectrum implies that positrons in the GaN bulk crystals annihilate either from a delocalized state in the lattice or as localized at vacancy defects. The positrons trapped at vacancies annihilate with the longer lifetime $\tau_{V}=\tau_{2}=235 \pm 5 \mathrm{ps}$. The decrease of the average lifetime at low temperatures indicates that the fraction $\eta_{V}$ of positron annihilations at vacancies decreases. When $\eta_{V} \rightarrow 0$, the component $\tau_{1}$ approaches the lifetime value $\tau_{B}$ of delocalized positrons in the lattice. At $10 \mathrm{~K}$, one can see that $\tau_{1}=164 \pm 1$ ps and $\tau_{\mathrm{av}}=167 \mathrm{ps}$. The positron lifetime in the $\mathrm{GaN}$ lattice can be interpreted to lie between these values, i.e., $\tau_{B}=166 \pm 1$ ps.

By comparing to atomic superposition calculations that showed that $\mathrm{N}$ vacancies could not explain the increase in the lifetime of the trapped positrons, it was concluded that Ga-vacancy-related defects are responsible for the $\tau_{2}=$ 235 ps lifetime component. Doppler broadening measurements were also performed on these crystals, and early values for the $S$ and $W$ parameters specific to the Ga-vacancyrelated defects could be proposed. It should, of course, be 
noted that these values depend strongly on the experimental geometry and detector details. In that work the Ga-vacancy complexes found in the bulk GaN crystals and MOCVD $\mathrm{GaN}$ thin epilayers on sapphire were shown to be correlated with the presence of yellow luminescence often observed in $n$-type GaN.

The vacancy defects in GaN and III-nitrides have since their identification been studied with positron annihilation spectroscopy by many research groups, with a total paper count amounting to about 200 in the past 15 years. The Ga-vacancy-related defects are now known to be the dominant intrinsic acceptor-type (compensating) defects in both unintentionally and intentionally $n$-type doped $\mathrm{GaN}$, irrespective of the method of growth (Saarinen, 2000; Tuomisto, 2010). When $\mathrm{Mg}$ is a contaminant, it also compensates for the $n$-type conductivity (and at high intentional concentrations produces highly resistive and/or $p$-type $\mathrm{GaN}$ ). The compensating Ga-vacancy-related defects exhibit negative charge states, with transitions deep in the wideband gap of $\mathrm{GaN}$, and they have been shown to contribute to both nonradiative recombination processes and luminescent processes such as the parasitic yellow luminescence. A wide body of experimental and theoretical research exists on these defects (Neugebauer and Van de Walle, 1994, 1996; Kaufmann et al., 1999; Armitage et al., 2003; Chow et al., 2004; Limpijumnong and Van de Walle, 2004; Van de Walle and Neugebauer, 2004; Reshchikov and Morkoç, 2005).

A more detailed identification of the Ga-vacancy-related defects was obtained relatively recently, thanks to the combination of coincidence Doppler broadening experiments and state-of-the-art theoretical calculations (Hautakangas et al., 2006). Electron irradiation experiments (Tuomisto, Ranki et al., 2007) were used to produce isolated Ga vacancies, and the data could be compared to GaN samples grown by different methods. Figure 7 shows the electron momentum distribution ratio curve of the $\mathrm{Ga}$ vacancy in $2-\mathrm{MeV}$ electronirradiated GaN. The signal of a clean Ga vacancy was obtained by decomposing the original Doppler broadening spectrum by determining the fraction of annihilations of delocalized positrons $\eta_{V}=40 \%$ from the positron lifetime measurement. The calculated curve for the isolated $\mathrm{Ga}$ vacancy correlates well with the experimental one through the whole momentum range, supporting the identification of the isolated $\mathrm{Ga}$ vacancy. The main contribution in the range between 2 and 4 a.u. arises from annihilations with $\mathrm{Ga} 3 d$ electrons. The decrease in intensity at this momentum region is due to the reduced intensity of $\mathrm{Ga} 3 d$ electrons in a $\mathrm{Ga}$ vacancy. The good agreement at both valence and core electron regions manifests the accuracy and predictive power of the theoretical calculations.

Figure 20 shows experimental ratio curves also for other vacancy-related defects in representative $\mathrm{GaN}$ samples grown by different methods. The curves are not similar, indicating that different complexes can be distinguished. In the momentum range between 2 and 4 a.u. the data have clear order. The intensity of irradiated $\mathrm{GaN}$ is the lowest while the oxygendoped GaN has higher intensity. This effect can be attributed to oxygen surrounding the Ga vacancy in the defect complex: the $\mathrm{O}$ atom is smaller than $\mathrm{N}$ and thus contributes more at high electron momentum. The same behavior can be seen in

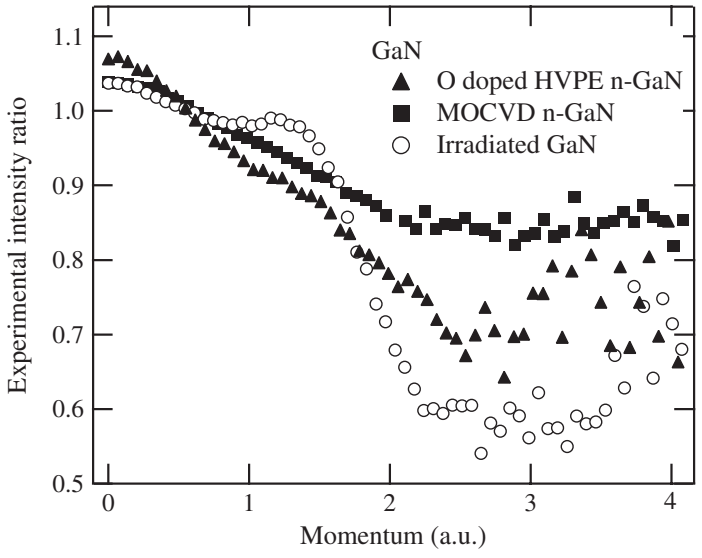

FIG. 20. Experimental coincidence Doppler ratio curves for irradiation-induced and two kinds of in-grown $\mathrm{Ga}$ vacancies. Adapted from Hautakangas et al., 2006.

the calculated momentum ratio curves. The difference between $V_{\mathrm{Ga}}-\mathrm{O}_{\mathrm{N}}$ and isolated $V_{\mathrm{Ga}}$ arises from the valence electron states derived from the atomic $2 p$ orbitals. The data in MOCVD GaN at a high-momentum region where the intensity is the highest of all samples are best explained by additional hydrogen in the $V_{\mathrm{Ga}}$ and $V_{\mathrm{Ga}}-\mathrm{O}_{\mathrm{N}}$ complexes. The contribution of hydrogen cannot be completely ruled out in the case of O-doped hydride vapor phase epitaxy GaN either, but in those samples it was also shown that the Ga-vacancy concentration is clearly correlated with the $\mathrm{O}$ concentration (Hautakangas et al., 2006). Lately the presence of vacancy-hydrogen complexes in MOCVD GaN has been observed to be directly related to nonradiative recombination (Nykänen et al., 2012).

\section{G. Metal vacancy-nitrogen vacancy complexes in III-nitrides and their alloys}

Metal vacancies and their complexes have been studied rather extensively in the III-nitrides and their alloys; see, e.g., Stampfl et al. (2000), Limpijumnong and Van de Walle (2004), Hautakangas et al. (2006), Duan and Stampfl (2008, 2009a, 2009b), Son et al. (2009), Van de Walle, Lyons, and Janotti (2010), Mäki, Makkonen et al. (2011), Rauch, Makkonen, and Tuomisto (2011), and Janotti, Lyons, and Van de Walle (2012). The vast majority of the studies are devoted to the binary compounds GaN, InN, and AlN, but some also make an attempt to understand the defect structure in ternary alloys. In fact, this is not a trivial task as the random alloys in principle exhibit a wide variety of local defect surroundings.

A systematic theoretical study of various defect spectra (ratio curves) in the case of $\mathrm{InN}$ is shown in Fig. 21 (Rauch, Makkonen, and Tuomisto, 2011). The ratio curve for the $V_{\text {In }}$ exhibits a distinct line shape with a maximum of $\sim 1.08$ at the peak center region ( 0 a.u.). For momenta above 0.6 a.u. the spectrum drops below 1 and a clear shoulder is visible at 1.2 a.u., which has been determined by atomic superposition calculations to stem from annihilations with $\mathrm{N} 2 p$ electrons. At $\sim 3.3$ a.u. a second broad peak appears with an intensity of $\sim 0.8$ relative to the $\mathrm{InN}$ lattice. Positron annihilation characteristics of the $2 V_{\text {In }}$ defect are very similar to those of the 


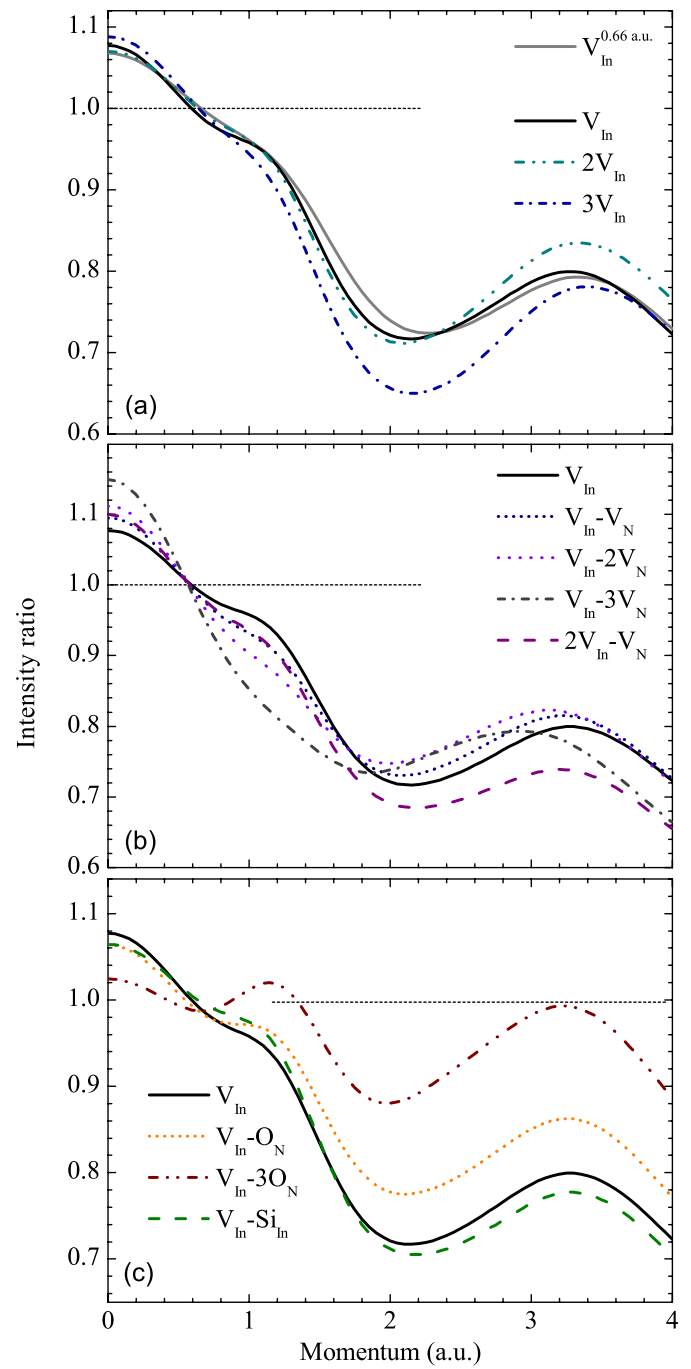

FIG. 21 (color online). Ratio curves of the calculated momentum densities of annihilating $e-p$ pairs in selected vacancy complexes in InN. All spectra are convoluted with a Gaussian of 0.53 a.u. FWHM (except $V_{\text {In }}^{0.66}$ a.u.,$F W H M=0.66$ a.u.) and divided by the momentum-density spectrum of the InN lattice. From Rauch, Makkonen, and Tuomisto, 2011.

isolated $V_{\text {In }}$. For the $3 V_{\text {In }}$ complex the ratio curve changes significantly with an increased peak maximum and a more pronounced drop at 2.1 a.u. Nevertheless, further analysis shows that the relative line shapes of the $3 V_{\text {In }}$ and $V_{\text {In }}$ are very similar and the spectrum of the $V_{\text {In }}$ can be essentially reproduced from the $3 V_{\text {In }}$ spectrum by assuming a positron annihilation fraction of $\eta \approx 0.8$. The $3 V_{\text {In }}$ and $V_{\text {In }}$ are therefore practically indistinguishable. Isolated $V_{\mathrm{N}}$ and pure $V_{\mathrm{N}}$ clusters do not localize positrons.

The calculated positron annihilation characteristics for the relaxed lattice structures of a variety of mixed vacancy complexes in InN, namely, $V_{\text {In }}-V_{\mathrm{N}}, V_{\mathrm{In}}-2 V_{\mathrm{N}}, 2 V_{\mathrm{In}}-V_{\mathrm{N}}$, and $V_{\mathrm{In}}-3 V_{\mathrm{N}}$ are shown in Fig. 21(b). A systematic trend compared to the isolated $V_{\text {In }}$ is visible in the spectra when adding an increasing amount of $V_{\mathrm{N}}$ around a single $V_{\text {In }}$. A strong increase of the zero momentum maximum to over 1.15 for the $V_{\mathrm{In}}-3 V_{\mathrm{N}}$ is visible, which is related to the increase in open volume. At the same time, the intensity of the shoulder at 1.2 a.u. decreases with an increasing number of $V_{\mathrm{N}}$ until it entirely disappears for
$V_{\text {In }}-3 V_{\mathrm{N}}$. The ratio curve of $2 V_{\mathrm{In}}-V_{\mathrm{N}}$ is close to that of $V_{\mathrm{In}}-V_{\mathrm{N}}$ for lower momentum values but starts to deviate at around 1.4 a.u. with lower intensities at higher momenta, due to reduced annihilation with In $4 d$ electrons. Figure 21(c) shows data for relaxed defect structures for the $V_{\text {In }}-O_{N}$, $V_{\mathrm{In}}-3 \mathrm{O}_{\mathrm{N}}$, and $V_{\mathrm{In}}-\mathrm{Si}_{\mathrm{In}}$ complexes. The peak maximum decreases with increasing number of $\mathrm{O}$ ions, while the intensity in the spectral range above 0.9 a.u. increases, including the shoulder at 1.2 a.u. and the peak at 3.4 a.u. The form of the $V_{\text {In }}-\mathrm{O}_{\mathrm{N}}$ ratio curve resembles the case of $V_{\text {In }}$ trapping with a reduced annihilation fraction of $\eta \approx 0.8$. The spectrum of the $V_{\text {In }}-\mathrm{Si}_{\text {In }}$ complex is very close to that of $V_{\text {In }}$ and hence hardly distinguishable in experiments. The case is different for the ratio curve of $V_{\mathrm{In}}-3 \mathrm{O}_{\mathrm{N}}$ which possesses distinct features with shoulders at 1.2 and 3.6 a.u., respectively, which should be measurable in coincidence Doppler measurements.

Figure 22 shows experimental ratio curves measured in selected InN samples. Sample I is a MBE-grown $\mathrm{InN}$

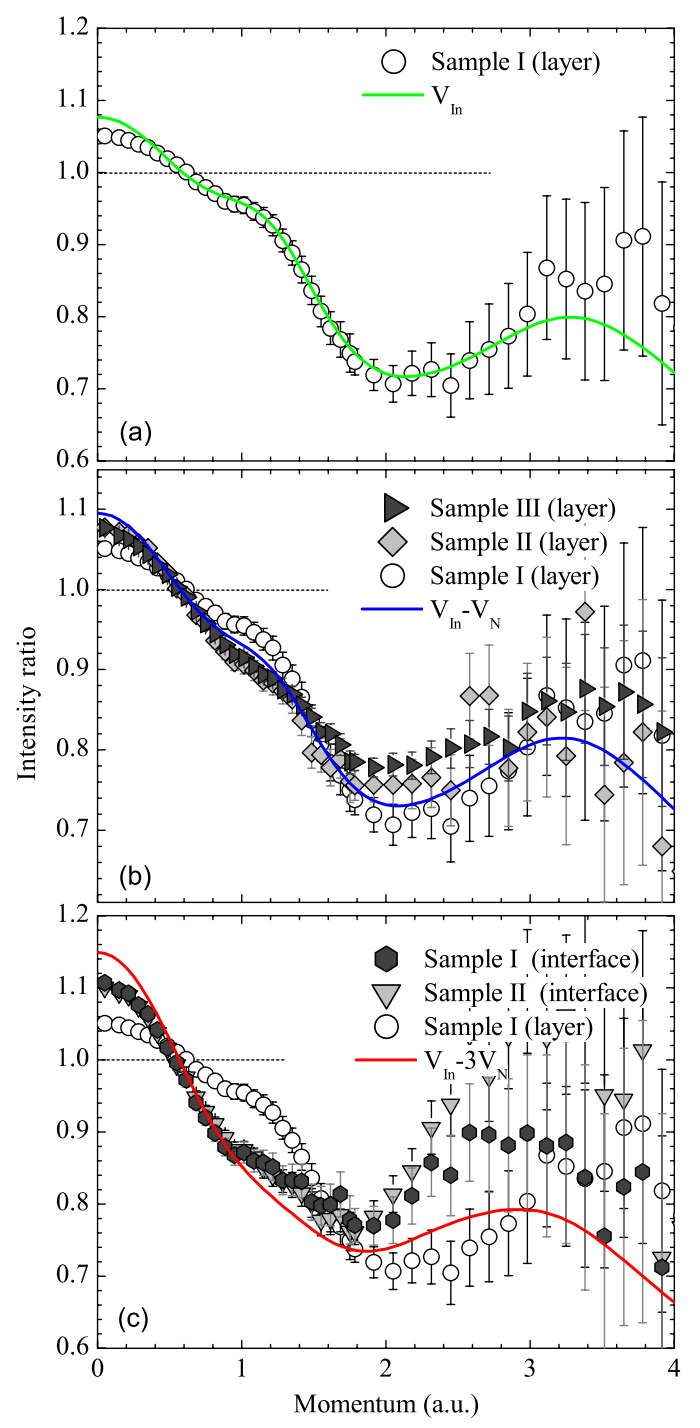

FIG. 22 (color online). Experimental coincidence Doppler spectra of the investigated samples in the layer (a), (b) and interface (c) region. The data have been divided by a suitable reference spectrum for the InN lattice. Computational ratio curves are shown for comparison. From Rauch, Makkonen, and Tuomisto, 2011. 
irradiated with 2-MeV He ions to a fluence of $9 \times 10^{15} \mathrm{~cm}^{-2}$; samples II and III are as-grown Si-doped and undoped InN layers deposited by MBE and MOCVD, respectively. Samples I and II show a strong change in the Doppler broadening signal when approaching the interface, a common feature in many InN samples. Therefore, the interface region is investigated separately in these two samples. The extrapolated ratio curve of sample I is shown in Fig. 22(a). This is in agreement with the spectrum of the isolated $V_{\text {In }}$ for most of the spectral range. In the central region of the peak slightly higher intensities are found in the calculated spectrum compared to the experimental one. This region is mostly sensitive to the size of the open volume of the positron trap, with higher intensities for larger volumes.

The extrapolated ratio curves of both sample II and the asmeasured spectrum of sample III show a very similar line shape in Fig. 22(b). The bigger scatter in the former is due to a smaller annihilation fraction. Compared to sample I, the asgrown samples II and III show several differences in their ratio curves. First, the intensity in the peak center region is clearly increased. The intensity difference from the InN lattice is thereby magnified by $\sim 35 \%$ compared to the spectrum of sample I. Second, a significant decrease of the shoulder at 1.2 a.u. is visible, also with high statistical accuracy. Third, the drop at 2 a.u. is less pronounced, followed by slightly higher intensities in the high-momentum region of the spectrum. A comparison with the calculated defect spectra in Fig. 21(b) reveals that these changes coincide with the effects of the decoration of a $V_{\text {In }}$ by $V_{\mathrm{N}}$. In particular, the characteristic decrease of the shoulder at 1.2 a.u. in ratio curves of the experimental spectra cannot be correlated with any other calculated vacancy defect complex.

At higher implantation energies strong changes in the Doppler broadening signal are observed for samples I and II, and the extrapolated ratio curves shown in Fig. 22(c). In both samples a strong increase in the peak center intensity to $\sim 1.12$ is visible, which is over twofold compared to that observed in the irradiated layer. Additionally, the signal drops straight to the minimum at 2 a.u. without showing any longer the shoulder which is visible in the layer region of both samples. The observed trends are qualitatively very similar to those of samples II and III, but are intensified. The induced changes can be associated with an increase in the decoration of $V_{\text {In }}$ with $V_{\mathrm{N}}$. When comparing to the calculated momentum distributions, the best agreement is found for the spectrum of the $V_{\text {In }}-3 V_{\mathrm{N}}$ complex.

The changes in the Doppler broadening spectra can naturally also be seen in the $(S, W)$ parameters. In particular, the decoration of the cation vacancies by the $\mathrm{N}$ vacancies is observed as a shift toward the right in the $(S, W)$ plane. This behavior has been observed in GaN (Hautakangas et al., 2006), InN (Rauch, Makkonen, and Tuomisto, 2011), and also the ternary alloys, and can be used to detect $V_{\mathrm{III}}-V_{\mathrm{N}}$ complexes. Figure 23 shows both experimental and theoretically predicted $(S, W)$ data in InGN samples (Uedono et al., 2012). These data, together with the data on $\mathrm{InN}$, have one remarkable feature that highlights the importance of performing both accurate experiments and state-of-the-art theoretical calculations. In elemental semiconductors and compound semiconductors with components of roughly the same size

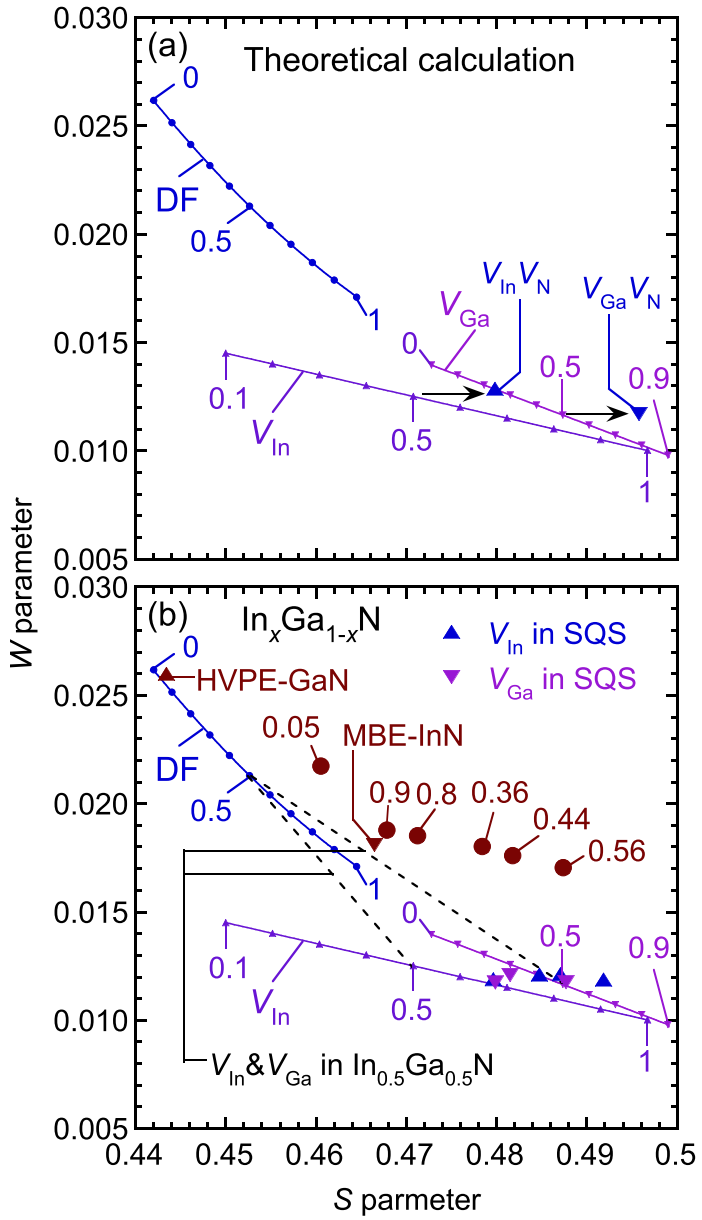

FIG. 23 (color online). (a) The $(S, W)$ values corresponding to the annihilation of positrons in the delocalized state (defect free, DF) and that of positrons trapped by cation vacancies $\left(V_{\mathrm{In}}\right.$ or $\left.V_{\mathrm{Ga}}\right)$ calculated using ordered $\operatorname{In}_{x} \mathrm{Ga}_{1 x} \mathrm{~N}(x=0, \ldots, 1$ with steps of $0.1)$. The $(S, W)$ values for $V_{\mathrm{In}} V_{\mathrm{N}}$ and $V_{\mathrm{Ga}} V_{\mathrm{N}}$ in $\mathrm{In}_{0.5} \mathrm{Ga}_{0.5} \mathrm{~N}$ are also shown. Arrows show the effect of $V_{\mathrm{N}}$ coupled with cation vacancies. (b) The $(S, W)$ value for the cation vacancies in SQS- $\operatorname{In}_{0.5} \mathrm{Ga}_{0.5} \mathrm{~N}$ [special-quasirandom structure SQS)] and experimentally obtained $S W$ relationship for $\operatorname{In}_{x} \mathrm{Ga}_{1 x} \mathrm{~N}$. The $x$ values for the sample are shown. The $(S, W)$ values for MBE-grown $\mathrm{GaN}$ and HVPE-grown GaN are also shown. The dotted lines connecting the $(S, W)$ values for DF and cation vacancies show the effect of the trapping of positrons by the cation vacancies in ordered $\operatorname{In}_{0.5} \mathrm{Ga}_{0.5} \mathrm{~N}$. From Uedono et al., 2012.

(such as GaAs), the positron lifetime is quite monotonically correlated with $S$ and $W$ parameters: an increase in lifetime means an increase in $S$ and a decrease in $W$. However, it seems that in strongly cation-anion-mismatched compounds, as exemplified by the results in $\mathrm{InN}$, cation-anion vacancy complexes can have very different $S$ parameters while producing similar positron lifetimes.

As an example, the predicted relative $S$ parameter specific to the $V_{\text {In }}-2 V_{\mathrm{N}}$ complex is $S=1.082\left(S=1.055\right.$ for $\left.V_{\text {In }}\right)$, a value often associated with very large vacancy complexes, while both are observed to exhibit the positron lifetime of a monovacancy-sized defect (the calculated lifetime for the two defects is the same within a couple of picoseconds). Hence comparisons with traditional knowledge acquired in $\mathrm{Si}$ and $\mathrm{GaAs}$ do not necessarily provide the best reference for 
interpretations in these kinds of compounds. Further, even if the different $V_{\text {In }}-n V_{\mathrm{N}}$ complexes give different points in the $(S, W)$ plot, the most dramatic difference is seen in the range between the $S$ and $W$ parameter windows. This highlights the importance of performing advanced theoretical calculations for detailed identification of defects.

\section{H. The substitutional lithium-on-zinc-site defect in zinc oxide}

As in the case of nitrogen vacancies in III-nitrides, it is often observed in calculations that small vacancy defects do not trap positrons. However, some cases bring surprises, such as, for example, the $\mathrm{Li}_{\mathrm{Zn}}$ defect. Figure 24 shows positron lifetime spectra recorded in two high-quality $\mathrm{ZnO}$ bulk crystals grown by the vapor phase (VP) and hydrothermal methods (HT) (Tuomisto et al., 2003; Johansen, Zubiaga, Makkonen et al., 2011). In both as-grown samples the positron lifetime spectrum has a single component: $170 \pm 1 \mathrm{ps}$ for VP and $184 \pm 1$ ps for HT. The value in the VP samples corresponds to positron annihilations in the defect-free lattice. The electron-irradiated VP sample has two lifetime components, the longer of which $\left(\tau_{2}=230 \mathrm{ps}\right)$ is due to positrons annihilating as trapped at in-grown $\mathrm{Zn}$ vacancies (Tuomisto et al., 2003; Tuomisto, Saarinen, Look, and Farlow, 2005).

The experimental results are often presented in terms of the average positron lifetime $\tau_{\text {av }}$ defined as the time expectation value of the experimental spectrum [see Eq. (25)], and it coincides with the center of mass of the spectrum. The latter property makes the average lifetime a statistically accurate parameter. Hence it can be correctly calculated from the intensity and lifetime values even if the decomposition represented only a good fit to the experimental data without any physical meaning. On the other hand, the decomposition is important, as, for example, when comparing the data in the as-grown HT sample and the electron-irradiated VP sample: the average positron lifetime is the same, but the spectra are

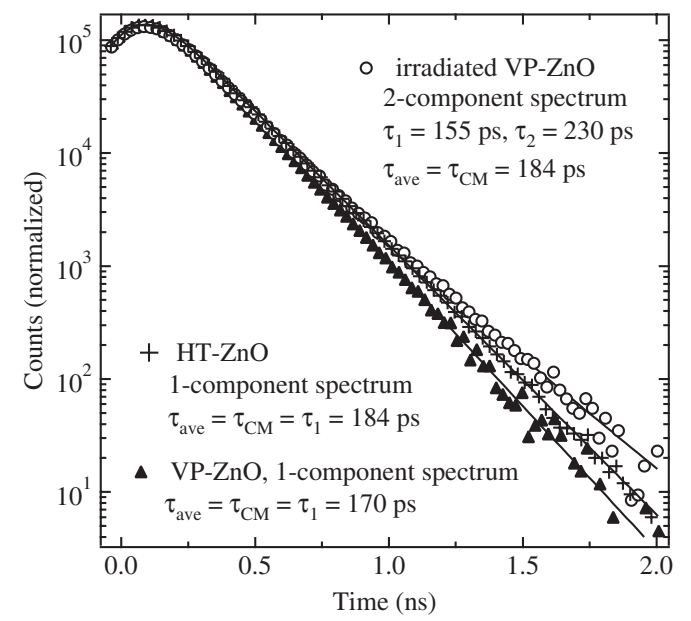

FIG. 24. Positron lifetime spectra measured for an electronirradiated VP-grown ZnO sample (Zubiaga et al., 2008), a typical spectrum for HT-grown $\mathrm{ZnO}$ (as well as for Li-enriched MG-grown $\mathrm{ZnO}$ samples) and the bulk lifetime spectrum as measured in the VP-grown $\mathrm{ZnO}$ reference sample. From Johansen, Zubiaga, Makkonen et al., 2011. clearly different (Fig. 24). In the HT sample the longer lifetime is caused by $\mathrm{Li}_{\mathrm{Zn}}$ whose defect-specific lifetime is very close to the average positron lifetime in the sample (Johansen, Zubiaga, Makkonen et al., 2011), while in the electronirradiated VP sample $\mathrm{Zn}$ vacancies are the cause of the increased lifetime.

The lifetime result for the HT-grown $\mathrm{ZnO}$ sample shown in Fig. 24 is the source of a wide scatter in reported $\mathrm{ZnO}$ lattice lifetimes. The reason for this is that in most HT-grown $\mathrm{ZnO}$ samples a single lifetime component is observed, but the values tend to be $10-15$ ps higher than, for example, in melt-grown (MG) $\mathrm{ZnO}$ or VP-grown $\mathrm{ZnO}$ (Puff et al., 1995; Brauer et al., 2007; Chen et al., 2007; Tuomisto and Look, 2007). It was recently shown (Johansen, Zubiaga, Makkonen et al., 2011) that this lifetime component, which is rather close to the $\mathrm{ZnO}$ lattice lifetime, is in fact related to Li impurities present in high concentrations in typical HTgrown $\mathrm{ZnO}$. $\mathrm{Li}$ on the $\mathrm{Zn}$ site $\left(\mathrm{Li}_{\mathrm{Zn}}\right)$ is theoretically predicted to be the stable form of $\mathrm{Li}$ in $n$-type $\mathrm{ZnO}$ (Wardle, Goss, and Briddon, 2005; Carvalho et al., 2009), and indeed state-ofthe-art calculations show that positrons can be trapped at $\mathrm{Li}_{\mathrm{Zn}}$ (i.e., the positron density is strongly localized at the defect), producing a lifetime 6-8 ps longer than in the $\mathrm{ZnO}$ lattice (Johansen, Zubiaga, Makkonen et al., 2011). This result is in fact slightly surprising, as it is generally thought that positron localization in a deep state requires at least a monovacancysized open volume. This result suggests that the observed trapping could be possible also in other cases where the $Z$ of the substitutional atom is much smaller than that of the host atom. In other words, from the positron point of view $\mathrm{Li}_{\mathrm{Zn}}$ is essentially $V_{\mathrm{Zn}}$ decorated by Li.

The case for the $\mathrm{Li}_{Z \mathrm{Zn}}$ model becomes much stronger when one considers the coincidence Doppler broadening data obtained in various samples. Figure 25 shows the ratio curves measured in the HT-grown and Li in-diffused MG samples

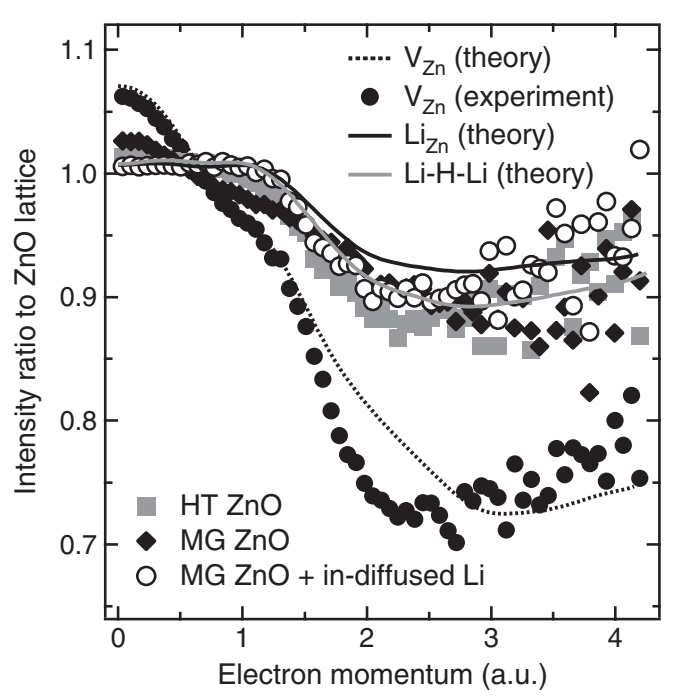

FIG. 25. Coincidence Doppler broadening measurements for as-grown $\mathrm{HT} \mathrm{ZnO}$, as-grown $\mathrm{MG} \mathrm{ZnO}$, and $\mathrm{Li}$-doped $\mathrm{MG} \mathrm{ZnO}$ as compared to the theoretical values obtained for $V_{\mathrm{Zn}}, \mathrm{Li}_{\mathrm{Zn}}$, and the $\mathrm{Li}_{\mathrm{Zn}}-\mathrm{H}-\mathrm{Li}_{\mathrm{Zn}}$ complex. The experimental data obtained for $V_{\mathrm{Zn}}$ are shown for comparison. From Johansen, Zubiaga, Makkonen et al., 2011. 
(normalized to the spectrum measured for the VP reference), together with the corresponding ratio curves obtained theoretically for $V_{\mathrm{Zn}}$, substitutional $\mathrm{Li}_{\mathrm{Zn}}$, and the $\mathrm{Li}_{\mathrm{Zn}}-\mathrm{H}-\mathrm{Li}_{\mathrm{Zn}}$ complex proposed by Sann et al. (2006). Also the $\mathrm{OH}-\mathrm{Li}_{\mathrm{Zn}}$ and $\mathrm{Li}_{\mathrm{Zn}}-\mathrm{Li}_{i}$ complexes have been calculated, using the ground-state configurations predicted by Wardle, Goss, and Briddon (2005), but they are not shown in Fig. 25 as the $\mathrm{Li}_{\mathrm{Zn}}-\mathrm{Li}_{i}$ pair is not found to be active as a positron trap while the curve for $\mathrm{OH}-\mathrm{Li}_{\mathrm{Zn}}$ is indistinguishable from the one for $\mathrm{Li}_{\mathrm{Zn}}$.

For comparison, Fig. 25 also includes data obtained for an irradiated sample with a high concentration of $V_{\mathrm{Zn}}$, illustrating that the features of the experimental and theoretical ratio curves (such as the shoulder at 1-1.2 a.u.) agree very well. From this, it is evident that the experimental ratio curves obtained in the Li-rich materials cannot be explained by assuming nonsaturated trapping by $V_{\mathrm{Zn}}$. On the other hand, the theoretical curves for the $\mathrm{Li}_{\mathrm{Zn}}, \mathrm{Li}_{\mathrm{Zn}}-\mathrm{H}-\mathrm{Li} \mathrm{Zn}_{\mathrm{Zn}}$, and $\mathrm{OH}-\mathrm{Li}_{\mathrm{Zn}}$ complexes all show excellent agreement with the data obtained from the Li-indiffused MG sample (the as-grown MG sample has some near-surface $\mathrm{Zn}$ vacancy defects). The differences between the $V_{\mathrm{Zn}}$ and $\mathrm{Li}_{\mathrm{Zn}}$ ratio curves are largely explained by the smaller open volume seen by the positron in the latter case. Li repels the positron toward neighboring ion cores, thereby increasing the high-momentum intensity relative to the $V_{\mathrm{Zn}}$ spectrum. The direct contribution of the $\mathrm{Li}$ orbitals to the $\mathrm{Li}_{\mathrm{Zn}}$ spectrum can be quantified by considering the system as a superposition of free atoms and decomposing the total annihilation rate to contributions due to different atomic orbitals. The Li contribution turns out to be only $5 \%$ of the total annihilation rate. Furthermore, the direct $\mathrm{Li}$ contribution to the Doppler spectrum is rather featureless. In conclusion, these calculations indicate that there is no clear "Li fingerprint," which would provide the possibility to unambiguously identify Li-related defects in a more general case. However, the flat region with the ratio slightly above 1.0 extending from 0 to 1.3 a.u. is unique for $\mathrm{Li}_{\mathrm{Zn}}$. It is evident from the data that $\mathrm{Li}_{\mathrm{Zn}}$ occurs as the dominant trap also in HT-grown $\mathrm{ZnO}$, but with a detectable contribution from $V_{\mathrm{Zn}}$.

Importantly, all $\mathrm{Li}$ atoms present in $n$-type $\mathrm{HT} \mathrm{ZnO}$ reside on the $\mathrm{Zn}$ site and the resulting open volume is thus responsible for the increase in the single-component positron lifetime observed in as-grown HT ZnO as compared to samples produced by other growth techniques yielding material with low $\mathrm{Li}$ concentrations. This also explains the discrepancy in the reported values for the bulk positron lifetime in $\mathrm{ZnO}$. It should be noted that the Li-related signal has been observed to disappear when the samples are hydrogenated (Johansen, Zubiaga, Tuomisto et al., 2011) suggesting that $\mathrm{Li}_{\mathrm{Zn}}$ traps hydrogen, turns neutral, and becomes less attractive to positrons. At the same time the remaining $V_{\mathrm{Zn}}$ defects have been shown to become efficient hydrogen traps. Further work is necessary in order to fully elucidate the role of residual hydrogen impurities and their interaction with intrinsic open-volume defects in $\mathrm{ZnO}$. Be in $\mathrm{GaN}$ may behave similarly (Lee et al., 2006; Lany and Zunger, 2010)

\section{FUTURE CHALLENGES}

Even if the methods presented in this review allow for advanced and detailed identification of certain types of vacancy defects in semiconductor materials (crystalline solids in general), there is a wide variety of important developments to be realized in order to unleash the full potential of positron annihilation spectroscopy in materials research. We have chosen five "challenges," in both the development of theoretical and experimental methods, which we find to be the most promising regarding our own interests. By no means is this meant as an exhaustive listing of all possible (or probable) developments in the field.

The first challenge is in studying electronic materials with complex crystal structures, such as complex oxides (Sec. V.A). In these materials the main issue is the large number of different lattice sites for monovacancy defects, making the defect identification complicated. The two further challenges concern the development of theoretical methods that would be crucial for detailed interpretation of experimental results in systems where the 3D periodicity is broken, namely, surfaces and interfaces between crystalline solids (Sec. V.B), and nanocrystalline, amorphous, and molecular systems (Sec. V.C). Reports on positron experiments in these areas are becoming more and more numerous, while the theoretical descriptions of positron states, thermalization, and trapping are not well established. The two last challenges concern developments in experimental methods: setups allowing for advanced sample state manipulation eventually allowing pump-probe experiments with light, bias, magnetic field, temperature, and pressure as the pump (Sec. V.D); and the issue of slow-positron beam intensity that can be solved by large-scale facilities providing intense sources or by improving positron moderation efficiency (retaining the quality) in laboratory-scale facilities (Sec. V.E).

\section{A. Materials with complex crystal structures}

Already a relatively moderate additional degree of complexity in the crystalline structure of a semiconductor material creates challenges in defect identification. This is true in general as well, but holds particularly for positron annihilation spectroscopy. In the following we discuss two kinds of complex crystal structures: multielement compounds and semiconductor alloys (or mixed crystals). The first are strictly periodic, but characterized by relatively large unit cells. The second are characterized by nonperiodic (with various degrees of randomness) distribution of atoms on the lattice sites.

By multielement compounds we mean all compound semiconductors that are more complex than just $A B$. Examples include materials with chalcogenide structure (such as $\mathrm{ZnGeAs}_{2}$ ) and complex oxides (such as $\mathrm{SrTiO}_{3}$ ). Some twocomponent compounds also fall into this category due to their very large unit cells: examples include $\mathrm{In}_{2} \mathrm{O}_{3}$ and the majority of $\mathrm{SiC}$ polytypes (see the discussion in Sec. IV.A.3). Positron annihilation has been employed to investigate defects in these kinds of materials; see, e.g., Niki et al. (2001), Uedono et al. (2002), Cheung et al. (2007), Keeble et al. (2007), Kilanski et al. (2009), Mackie et al. (2009), Gentils et al. (2010), Islam et al. (2011), Guagliardo et al. (2012), and Korhonen et al. (2012). While the results are promising and pave the way for future studies, systematic studies are still missing.

The main challenge in identifying vacancy defects in these multielement compounds is the large number of possible 
vacancy-type defects already for monovacancies. This in contrast to elemental semiconductors (for which the number is 1) and simple compounds such as GaAs (for which the number is 2). Often for these kinds of simple systems one kind of vacancy defect dominates, making the identification with positron annihilation methods relatively straightforward, as seen in the discussions in the previous section. For multielement compounds, the definitions of stoichiometry and chemical potential are much more complicated, and it is less probable that in a given sample there would be one dominant kind of defect-this is seen, in particular, in the studies on chalcogenide-structured materials (Niki et al., 2001; Kilanski et al., 2009; Islam et al., 2011; Korhonen et al., 2012). In addition to the three possible monovacancies in such materials, the number of possible binary complexes of intrinsic defects only is strongly increased compared to simple structures (e.g., divacancies, vacancy-antisite complexes). In "simple" complex oxides such as $\mathrm{SnO}_{2}$ or $\mathrm{ZrO}_{2}$ (Guagliardo et al., 2012) the situation is not as bad as in the multielement compounds, but the possibility of metal vacancy-oxygen vacancy complexes with multiple oxygen vacancies makes detailed defect identification difficult. For multimetal complex oxides (Uedono et al., 2002; Cheung et al., 2007; Keeble et al., 2007; Mackie et al., 2009; Gentils et al., 2010) these two challenges are combined, making identification even more difficult. These challenges should, however, be overcome by systematic studies where state-ofthe-art theoretical calculations and careful experiments are combined. Significant efforts by several actors in the field can be anticipated, and the challenge of detecting $\mathrm{O}$ vacancies in complex oxides should be highlighted.

Semiconductor alloys, such as $\mathrm{Si}_{1-x} \mathrm{Ge}_{x}$ and $\mathrm{In}_{x} \mathrm{Ga}_{1-x} \mathrm{~N}$, possess another complication that is harder to tackle, especially when $x$ is significantly different from either 0 or 1 . Combining theoretical calculations and experiments is conceptually more complicated. As an example, in a calculation one knows whether an In or $\mathrm{Ga}$ atom has been removed to make a vacancy on the cation sublattice in $\operatorname{In}_{x} \mathrm{Ga}_{1-x} \mathrm{~N}$, but in experiments the positrons are primarily sensitive to the "cation vacancy" (Chichibu et al., 2006, 2011, 2013; Uedono et al., 2009, 2012). Hence the second-nearest-neighbor environment becomes very important in defect identification. This phenomenon is yet to be studied in detail. There is significant room for improvement, but here it might not be sufficient to apply existing, even if state-of-the-art, theoretical methods to account for the randomness. The importance of the effects of the local environment of the vacancy defects being identified compared to the long- range disorder need to be elucidated (Kuitunen, Tuomisto, and Slotte, 2007; Kilpeläinen et al., 2010, 2011). Systematic studies in all these materials are required in order to fully elucidate the vacancy defect identities and roles.

\section{B. Positron states at interfaces and surfaces}

In order to understand positron annihilation parameters measured for such complex systems as semiconductor alloys or heterostructures, the first question one has to address, preferably with the help of computational modeling, is which kinds of regions the positron will be likely to sample. In other words, what is the "affinity" of positrons for, for instance, GaN or InN clusters in InGaN alloys [see, e.g., Chichibu et al. (2006)], or for different layers in quantum wells or superlattices formed of these materials? Further, what is the effect of polarization in polar semiconductor heterostructures from the point of view of positron studies?

On the other hand, a requirement for studying surfaces with positrons or for the construction of efficient positron moderators (Sec. V.E) is an understanding of the positron surface state. For instance, one wants to know how to best modify the moderator's surface in order to obtain high emission efficiency from the bound state into the vacuum. The interaction between the positron and the surface is not easy to model. In addition, the image potential sensed by the positron above a conducting surface is a highly nonlocal correlation effect, especially when positronium formation is also expected and the van der Waals interaction plays a role. Currently effects such as this can be modeled using only simple models [see, e.g., Saniz et al. (2007, 2008) and Mukherjee et al. (2010)]. More understanding and quantitative modeling is needed in this area as well.

The concepts of material-specific positron affinity and the positron affinity difference determining the separation of positron energy levels between two solids in contact (Boev, Puska, and Nieminen, 1987; Puska, Lanki, and Nieminen, 1989) are based on a model strictly speaking valid only for metals. In the model, it is assumed that the Fermi levels equalize themselves via charge transfer and formation of an interface dipole $\Delta=\mu_{-}^{A}-\mu_{-}^{B}$, where $\mu_{-}^{A, B}$ are the electron chemical potentials for materials $A$ and $B$ while separated. Then, the difference between positron energy levels $E_{+}$on the different sides of the interface is (Puska, Lanki, and Nieminen, 1989)

$$
\begin{aligned}
\Delta E_{+}^{A, B} & =E_{+}^{A}-E_{+}^{B}=\Delta+\mu_{+}^{A}-\mu_{+}^{B} \\
& =\mu_{-}^{A}-\mu_{-}^{B}+\mu_{+}^{A}-\mu_{+}^{B},
\end{aligned}
$$

where $\mu_{+}^{A, B}$ are the chemical potentials for the positron. As a consequence, it is useful to define a bulk property $A_{+}=\mu_{-}+\mu_{+}$, the positron affinity, and calculate the difference in positron energies using the difference of the positron affinities. However, if one or both of the materials are semiconductors, and the Fermi level is aligned within the band gap, there are no extended electronic states to accommodate the charge on the semiconductor side. However, there may exist localized states at the interface but these cannot be predicted using bulk properties only. First-principles modeling of the interfaces themselves is needed [see the related discussion by Van de Walle, Lyons, and Janotti (2010)]. Further, creation of an interface always involves strain which also affects the positron energy (Boev, Puska, and Nieminen, 1987; Puska, Lanki, and Nieminen, 1989) levels as well as electron band alignments [see, e.g., Moses et al. (2011)].

Another reason why first-principles calculations (beyond the atomic superposition method) should be used throughout for modeling positron states and annihilation in semiconductor structures is that in addition to the band gap modulation and variation of positron energy levels, there may exist huge macroscopic electric fields in polar semiconductor heterostructures and superlattices (Bernardini and Fiorentini, 1998; Fiorentini et al., 1999; Lefebvre et al., 2001). In a classical 
model (Fiorentini et al., 1999), a discontinuity of the transverse polarization at an interface corresponds to an interface charge. Once again, the detailed electronic structure of the interface determines how much charge it actually can accommodate, and first-principles calculations are a necessity. Similarly, the model does not account for the screening of the macroscopic electric field when the potential difference across a heterostructure's layer becomes larger than the energy band gap of the material.

Makkonen et al. (2010) modeled the behavior of positrons in nonpolar and polar superlattices composed of GaN, AlN, and InN using first-principles methods. For nonpolar superlattice models it is observed that the separation of the positron energy levels depends on strain. In the case of polar superlattice models, the macroscopic electric field drives the positron density toward one of the two inequivalent interfaces in the models. Although cation vacancies situated at the nitride layers are expected to be energetically more favorable than this positron's interface state, the trapping rate to the vacancies is expected to decrease with increasing distance of the vacancy from the preferred interface. This is expected to provide interface sensitivity in positron measurements made for such structures.

In conclusion, understanding positron annihilation results measured for semiconductor heterostructures requires consideration and ideally supporting first-principles modeling of positron states and annihilation to understand where the annihilation signal is coming from and the indirect information contained in the data. The electronic structure has to be modeled self-consistently and models should include detailed structures of the interfaces. In the case of systems in which the common LDA and GGA exchange and correlation functionals underestimate the energy band gap, one benefits from the use of hybrid exchange-correlation functionals (Becke, 1993; Perdew, Ernzerhof, and Burke, 1996; Adamo and Barone, 1999; Heyd, Scuseria, and Ernzerhof, 2003) for the description of the electronic structure, although the computational cost is then significantly higher.

\section{Positron thermalization and trapping in nanocrystalline, amorphous, and molecular systems}

Positron annihilation spectroscopy can be used to study properties of nanoscale structures and their surfaces, either nanocrystalline [see, e.g., Weber et al. (2002) and Eijt et al. (2006)] or as embedded in the bulk of the material (Nagai et al., 2000; Chichibu et al., 2006). The sizes of nanocrystals can be correlated with smearing effects of various origins observed in measured momentum densities (Saniz, Barbiellini, and Denison, 2002; Weber et al., 2002; Toyama et al., 2012).

In a typical positron experiment, it is assumed that the positrons thermalize very rapidly within a few picoseconds. Then the time-dependent diffusion equation and the conventional trapping model (see Sec. II.A.3) can be applied to describe the diffusion and trapping kinetics of positrons. According to Jensen and Walker (1990), measurable deviations from the conventional trapping model will happen only if the trapping rate and/or annihilation rate differ from the thermal rate for a sufficient fraction of the thermalization period. Effects of incomplete thermalization of positrons have been observed experimentally for semiconductors and insulators (Mills and Crane, 1985; Gullikson and Mills, 1986; Lynn and Nielsen, 1987; Nissilä, Saarinen, and Hautojärvi, 2001) and even for metals (Nielsen, Lynn, and Chen, 1986; Huomo et al., 1987). In bulk studies with energetic positrons, it is usually safe to neglect nonthermal effects. However, if one is measuring nanocrystalline samples, it might happen that the positrons leave the crystal already at nonthermal energies. If the nonthermal trapping rate differs from the thermal rate, the conventional trapping model is not applicable. A resonant-trapping mechanism effective at nonthermal energies has been proposed for metals (McMullen and Stott, 1986; Puska and Manninen, 1987; Jensen and Walker, 1990) and similar mechanisms also play a role in semiconductors (Puska, Corbel, and Nieminen, 1990), and especially in trapping to vacancy clusters and voids in metals (Puska and Manninen, 1987; Jensen and Walker, 1992). Also, even if the positron does end up thermalized within a nanocrystal it might not possess a well-defined delocalized "bulk state" if the material has a negative positron work function and the nanocrystal's dimensions are smaller than the thermal wavelength $^{4}$ of the positron.

In order to fully understand positron thermalization and trapping into various kinds of states (vacancy, void, interface, or surface state) in nanocrystals, one needs to address transition rates between different states including also the possibility of nonthermal and resonant effects, and model the thermalization and trapping processes using a model including both spatial and momentum transport and having realistic geometries for the nanocrystals. Further, more understanding of thermalization and trapping mechanisms and their effectiveness in not only nanocrystalline but also amorphous and molecular matter is needed on a general level. In soft and molecular matter, the most important information in the annihilation signal is often contained in the pick-off annihilation of ortho-Ps (the positron within triplet positronium annihilating with outside electrons); see, e.g., Tao (1972), Eldrup, Lightbody, and Sherwood (1981), Jean (1990), Hirata, Kobayashi, and Ujihira (1996), Dong et al. (2009), Sane et al. (2009), and Quinn et al. (2012). This correlated state involving also nonlocal correlations (van der Waals interaction) with the surroundings is extremely challenging to model for any realistic system [see Barbiellini and Platzman (2009) and Zubiaga, Tuomisto, and Puska (2012)].

Related to the above we note that the trapping of positrons in vacancy defects even in crystalline semiconductor systems poses challenges for the present theoretical models when the energetics of trapping is considered (Makkonen and Puska, 2007). An important difference between vacancies in metals and semiconductors is the lower positron binding energy expected for the latter. This is because of the stronger repulsive interaction between a delocalized positron and nuclei in the denser structures of metals. Consequently, the lowering of

\footnotetext{
${ }^{4}$ The thermal wavelength can be estimated as $\lambda_{\mathrm{th}}^{+}=\frac{h}{\sqrt{3 m^{*} k_{B} T}} \approx 50\left(\frac{300 \mathrm{~K}}{T}\right)^{1 / 2} \AA$.
} 
the positron's energy eigenvalue between the bulk state and the trapped state at a semiconductor vacancy is not necessarily much larger in magnitude than the energy stored in the accompanying ionic relaxation [see the terms in Eq. (40)]. Calculations even suggest that the bound state resembles the case of an electron or a hole trapped into a small polaron state in ionic crystals. According to present theoretical models, the trapping could not even be energetically favorable in some important materials systems ( $\mathrm{Si}, \mathrm{Ge}, \mathrm{GaAs}$ ) or then an energy minimum (even a metastable one) with a trapped positron would not exist (Makkonen and Puska, 2007). These predictions are not supported by the experimental observation in which trapping to the same defects is observed, and possible temperature dependences are due to other mechanisms. When interpreting experiments especially in new materials, the starting point is often in trying to understand which defects can trap positrons. Potential borderline cases continue to pose an important challenge for theoretical models.

\section{Pump-probe experiments with positron annihilation spectroscopy}

The state of the art of positron annihilation spectroscopy experiments in studying defects in semiconductors is based on the control of sample temperature during measurements in typical ranges of $10-600 \mathrm{~K}$, providing information on the equilibrium charge states of the detected defects, and making it possible to identify the electronically important defects. Sample illumination with sub-band-gap monochromatic light during experiments brings additional data on optical charge transitions of these defects, relating them to optoelectronic properties of semiconductors. However, in order to directly relate the vacancy defects that can be identified with positron annihilation spectroscopy to carrier dynamics (e.g., nonradiative defect-related recombination processes) directly affecting the function of optoelectronic devices, more sophisticated experiments need to be thought of. One step in this direction is to time modulate the illumination: Fig. 26 shows a recent result obtained in natural diamond with modulated illumination, where the recombination process happens to be slow enough (seconds to hundreds of seconds) for the effects to

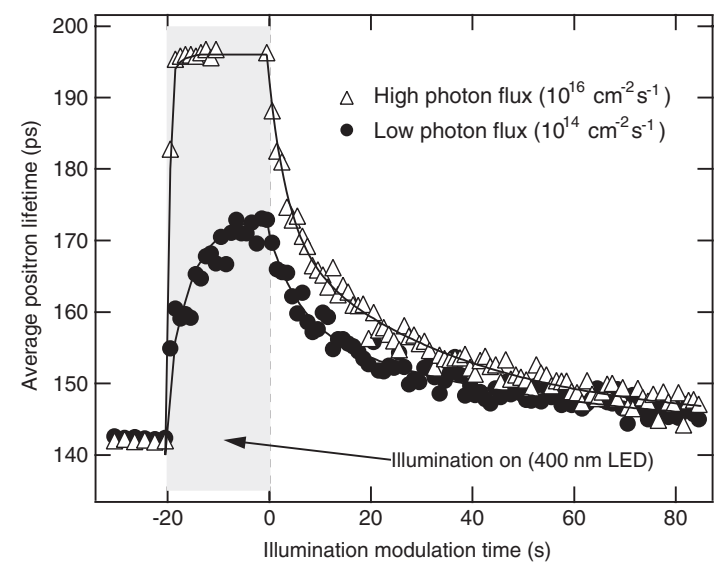

FIG. 26. Example of average positron lifetime measured with time-modulated illumination in a natural diamond sample. be monitored by usual experiments (Mäki, Tuomisto et al., 2011; Mäki et al., 2012). Typically in semiconductor materials the recombination rates are several orders of magnitude shorter (down to the nanosecond scale); hence further development is required.

In the example shown in Fig. 26, the sample-sourcesample sandwich was illuminated with high-power lightemitting diodes (LEDs), as the illumination intensity can be a bottleneck when maximal ionization efficiency is needed. In steady state (during illumination) the fraction of ionized defects will depend on their optical absorption cross section, and the relaxation rate of the latter may be quite fast especially at elevated temperatures. By controlling the LEDs with a fast electrical switch, the illumination can be changed from steady state to transient mode, allowing pump-probe experiments. In these experiments the collection of positron annihilation data can be divided into time slots (1-10 s in the example experiment), both during and after the illumination pulse. By repeating the measurement several hundred times, the data collected in each time slot can be summed and time-dependent positron lifetime spectra obtained. The experimental data in Fig. 26 show that in a case when both the rising time and the relaxation time of the illumination effect are slow (LED response is in the microsecond regime, so the effect comes from the studied material), the positron experiments can be used to follow the population and depopulation of vacancy defect levels. Combining optical absorption experiments, illuminationpower-dependent steady-state positron experiments and these pump-probe positron experiments allow one to selfconsistently determine the optical absorption cross section and the vacancy concentrations, without prior knowledge of the positron trapping coefficient (Mäki, Tuomisto et al., 2011).

These experiments are naturally not restricted to diamond or a specific wavelength of light. It is sufficient that the vacancy defects detected with positrons have electron levels in the band gap of the semiconductors and can be optically ionized. A good example is given in Sec. IV.D where the EL2 defect studies in GaAs are reviewed. In addition, electrons can be excited to deep levels in the gap also by electrical (bias) pulses, in a manner similar to DLTS. Here only nearsurface phenomena can be monitored, so the method would be applicable only with slow-positron beams. Also thin metal contacts on the samples to be studied are necessary. There is no obstacle in the realization of these kinds of experiments, as there are several reports of measurements of biased samples for determining positron mobility in semiconductors (Simpson et al., 1990; Mäkinen et al., 1991). With simple setups for pump-probe experiments [such as the one described by Mäki et al. (2012)], one can easily go down to the microsecond regime in time scales, although new data analysis techniques are needed: Continuous measurements with varying illumination pulse lengths and periods should be performed as the collection of data in microsecond-range time slots is not feasible. Further reduction of the time scale down to the nanosecond range is possible by synchronizing the excitation with the time modulation of a pulsed positron beam, where the pulsing periods are typically in tens of nanoseconds. 
Application of a strong (varying) magnetic field to samples during positron experiments is also an option, but in many cases it has severe effects on the detection of the signal itself, as (i) the positrons are magnetically guided to and focused on the sample in a slow-positron beam, and (ii) the photomultiplier tube(s) in positron lifetime experiments are extremely sensitive to magnetic fields. Performing Doppler broadening (and ACAR) experiments in bulk crystals in varying magnetic fields has been realized and effects on the positron signals reported (Kawasuso et al., 2011, 2012). Theoretical calculations also predict observable changes for certain types of defects in semiconductors (Alatalo, Puska, and Nieminen, 1993). Hence developments in this direction should also be pursued. In the case of lifetime experiments, technology also poses a challenge: the time resolution and efficiency requirements are such that present-day avalanche photodiodes (APDs, the reasonable alternative for photomultiplier tubes) are not applicable. Recent results on AlGaN APDs are, however, encouraging (Sun et al., 2010).

\section{E. Toward higher slow-positron beam intensity}

In the field of condensed matter and materials physics, the vast majority of slow-positron beams is used for experiments on the Doppler broadening of the positron-electron annihilation radiation. Other techniques, i.e., positron lifetime spectroscopy, ACAR, PAES, and RHEPD, require either sophisticated beam pulsing electronics (or other timing technology), or high-intensity sources, or both. Hence these other techniques are currently restricted to a handful of large-scale facilities, although pulsed positron beams have been constructed at laboratory-scale facilities, too.

The laboratory-scale facilities typically employ radioactive $\left(\beta^{+}\right)$isotopes, such as ${ }^{22} \mathrm{Na}$ that has relatively low intensity (up to $10^{9}$ positrons/s). The low intensity is balanced by the practical half-life of 2.6 years allowing reasonable use of the same source for 6-10 years. On the other hand, large-scale facilities with high-intensity sources are able to provide up to $10^{12}$ positrons/s, making use of pair production with the high-energy gamma flux created by a nuclear reactor or a particle accelerator (Cassidy et al., 2009; Krause-Rehberg et al., 2011). For more details about intense positron sources, see Hugenschmidt et al. (2004), Schut et al. (2004), and Hawari et al. (2009). High-intensity positron sources indeed represent the state of the art of experimental development, but require significant resources due to their large scale.

The present-day moderator solutions are using either a thin $\mathrm{W}$ foil (efficiency $\sim 10^{-4}$ ) or a solid Ne moderator (efficiency $\sim 10^{-3}$ ). The thin $\mathrm{W}$ foil moderator has relatively poor efficiency, but produces slow positrons with a narrow energy spectrum. The solid Ne moderator is better in efficiency, but produces positrons with a wider energy spectrum that makes it impractical for positron lifetime beams (Mills and Gullikson, 1986; Mills and Platzman, 2001). In addition, in the case of high-voltage floated positron sources (grounded sample stages for ease of sample state manipulation), the requirement of weekly regeneration and cryogenics on high-voltage platforms is not particularly appealing in the case of solid Ne moderators. Further energy selection and beam formation techniques reduce the beam intensity by an additional order of magnitude, hence resulting in actual (maximum) slow-positron beam intensities of $10^{4}$ and $10^{8} e^{+} / \mathrm{s}$ for laboratory-scale and large-scale facilities, respectively, when using the more common passive thin $\mathrm{W}$ foil moderation. Often the beam intensities are roughly an order of magnitude lower. Beam bunching and chopping further reduces the intensity in the case of pulsed positron beams.

There is significant room for improvement in the positron moderators in both their efficiency and the directional dispersion (exit cone of positrons) that both limit the beam intensity. The maximum realistic beam intensity for defect studies is of the order of $10^{8} e^{+} / \mathrm{s}$ (requirement of no positron-positron interactions), so the large-scale facilities are already close in that respect, but there is no practical upper limit for PAES and RHEPD beam intensities. Limitations are imposed also from the signal detection point of view: measurement times of much less than $100 \mathrm{~s}$ per spectrum are not feasible in the case of Doppler broadening, as the peak stabilization requires some time. Also the capability of lifetime experiments going above 1000 counts/s is limited (here the detection efficiency is much lower than in Doppler broadening experiments: in typical experimental configurations about $1 \%$ of all the annihilations are detected). The wide directional dispersion of the moderated positrons is partly responsible for the small number of SPMs, comparable to a SEM, as the focusing of the beam with reasonable intensity even at a large-scale facility results in spot sizes of the order of $5 \mu \mathrm{m}$.

Three factors determine the efficiency of a positron moderator: (i) the fraction of positrons stopped within the moderator, (ii) the fraction of stopped positrons reaching the moderator surface, and (iii) the fraction of surface-reaching positrons emitted from the surface (see Fig. 27). For conventional thin-film W moderators in the transmission geometry, the first is roughly 5\% and the second roughly 20\%; (these are fully determined by the thickness) and the quality (crystalline is best) of the film optimum is about $1 \mu \mathrm{m}$. The emission efficiency can be optimized by careful surface preparation through thermal treatments in high vacuum and through maintaining a good vacuum in the beam. However, it is very difficult to have an emission (extraction) efficiency above $1 \%$-hence the total efficiency is at best $10^{-4}$. The first two limiting factors can be significantly improved by using a semiconductor material (an order of magnitude thicker than $\mathrm{W}$, hence stopping more positrons) and applying a voltage across to improve the diffusion to the surface

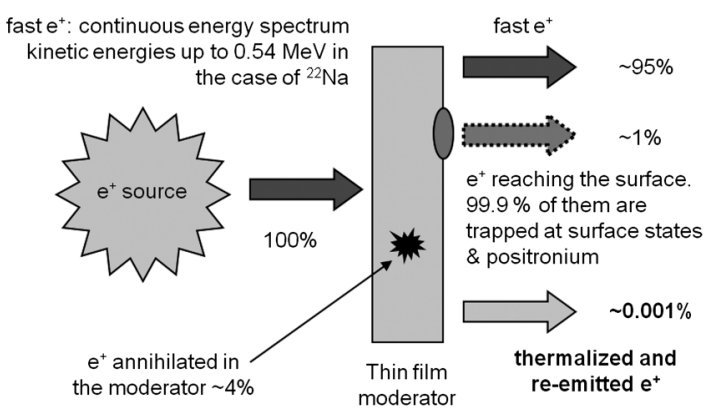

FIG. 27. Schematic of a thin-film moderator in transmission geometry. 
(Shan et al., 1994). By choosing, e.g., GaN, SiC, or diamond, the fraction of positrons reaching the extraction surface could be increased up to $50 \%$. These three materials also have a negative positron affinity (Coleman, 2000), and hence positron extraction from the surface should be possible. The remaining, important, challenge is in the nature of semiconductors: an electrical dipole is formed at the semiconductorvacuum interface, creating a strong positron trap preventing positron emission. The emphasis on the development in this area should be in the surface processing and passivation techniques (from the positron point of view) that will allow efficient positron extraction. The radiation hardness and mature semiconductor device technology in III-nitrides suggests that this material family could be the key to improved positron moderators.

Future improvements in moderator efficiencies would have important consequences: the significant reduction of measurement times significantly is important for both laboratory-scale and large-scale facilities. Of more general importance is that improved moderator efficiency will make the construction of a reasonable (i.e., reasonable intensity) isotope-source-based slow-positron beam much more accessible for a great number of laboratories. This is because one could achieve the present intensities (which are satisfactory for standard experiments) with a source whose activity is only $2 \mathrm{MBq}$, similar to widely used common fast-positron sources. Finally, there are direct experimental benefits from lower source activity, such as less background and noise in the experiments, and the beams can be constructed with much more compact dimensions (downscaling from $\sim 3 \mathrm{~m}$ beam line length to less than $1 \mathrm{~m}$ ). Improved beam intensities would clearly also benefit the pump-probe experiments described in Sec. V.D, as well as enable the efficient use of PAES and RHEPD. Also SPM could be used more efficiently in a scanning mode, mimicking the depth profiling typical of spreading resistance measurements (Krause-Rehberg et al., 2001).

\section{SUMMARY}

Positron annihilation spectroscopy is a characterization method for probing the local electron density and atomic structure at the site chosen by the electrostatic interaction of the positron with its environment. The positron annihilation methods have had a significant impact on defect spectroscopy in solids by introducing an experimental technique for the unambiguous identification of vacancies. Native vacancies have been identified and found to be present at high concentrations in many semiconductors, and their role in doping and compensation can be quantitatively discussed. Defect charge states and transitions, as well as their formation and migration processes, can be studied with positron methods.

We summarized the basic concepts behind the experimental and theoretical methods of positron annihilation and reviewed the latest developments that have led to the possibility of identifying defects in semiconductors with a high level of detail. We hope to provide useful reference material for the specialist and at the same time provide the nonpractitioner additional means to assess positron results and interpretations through a frank account of the strengths and weaknesses of the experimental and theoretical methods. The examples from various technologically important semiconductors illustrate the important combination of experiment and theory in detailed defect identification with positrons. Future challenges include the development of quantitative theoretical models for noncrystalline systems and the development of experimental arrangements enabling the analysis of defect-related transient phenomena.

\section{ACKNOWLEDGMENTS}

We thank the Academy of Finland, Helsinki Institute of Physics and Aalto University for financial support. We are particularly thankful to B. Barbiellini, Z.Q. Chen, P. G. Coleman, R. Krause-Rehberg, M. D. McCluskey, M. J. Puska, Z. Tang, and A. Uedono for their valuable suggestions and comments during the writing of this manuscript. We acknowledge the computational resources provided by the Aalto Science-IT project.

\section{REFERENCES}

Aavikko, R., K. Saarinen, F. Tuomisto, B. Magnusson, N. T. Son, and E. Janzén, 2007, Phys. Rev. B 75, 085208.

Adamo, C., and V. Barone, 1999, J. Chem. Phys. 110, 6158.

Alatalo, M., B. Barbiellini, M. Hakala, H. Kauppinen, T. Korhonen, M. J. Puska, K. Saarinen, P. Hautojärvi, and R. M. Nieminen, 1996, Phys. Rev. B 54, 2397.

Alatalo, M., H. Kauppinen, K. Saarinen, M. J. Puska, J. Mäkinen, P. Hautojärvi, and R. M. Nieminen, 1995, Phys. Rev. B 51, 4176.

Alatalo, M., M. J. Puska, and R. M. Nieminen, 1993, J. Phys. Condens. Matter 5, L307.

Ambigapathy, R., A. A. Manuel, P. Hautojärvi, K. Saarinen, and C. Corbel, 1994, Phys. Rev. B 50, 2188.

Andersen, O. K., 1975, Phys. Rev. B 12, 3060.

Andersen, O. K., O. Jepsen, and D. Glötzel, 1985, in Highlights of Condensed-Matter Theory, edited by F. Bassani, F. Fumi, and M. P. Tosi (North-Holland, Amsterdam).

Anderson, C.D., 1933 Phys. Rev. 43, 491.

Arifov, P. U., N. Y. Arutyunov, and A. Z. Ilyasov, 1977, Sov. Phys. Semicond. 11, 907.

Armitage, R., W. Hong, Q. Yang, H. Feick, J. Gebauer, E. R. Weber, S. Hautakangas, and K. Saarinen, 2003, Appl. Phys. Lett. 82, 3457.

Arpiainen, S., K. Saarinen, P. Hautojärvi, L. Henry, M.-F. Barthe, and C. Corbel, 2002, Phys. Rev. B 66, 075206.

Arponen, J., P. Hautojärvi, R. Nieminen, and E. Pajanne, 1973, J. Phys. F 3, 2092.

Arponen, J., and E. Pajanne, 1979a, J. Phys. F 9, 2359.

Arponen, J., and E. Pajanne, 1979b, Ann. Phys. (N.Y.) 121, 343.

Arutyunov, N., and V. Emtsev, 2007, Physica B (Amsterdam) 401-402, 609.

Asoka-Kumar, P., M. Alatalo, V.J. Ghosh, A.C. Kruseman, B. Nielsen, and K. G. Lynn, 1996, Phys. Rev. Lett. 77, 2097.

Asoka-Kumar, P., K. G. Lynn, and D. O. Welch, 1994, J. Appl. Phys. 76, 4935.

Avalos, V., and S. Dannefaer, 1996, Phys. Rev. B 54, 1724.

Bachelet, G. B., D. R. Hamann, and M. Schlüter, 1982, Phys. Rev. B 26, 4199.

Bansil, A., P. E. Mijnarends, and L. C. Smedskjaer, 1991, Phys. Rev. B 43, 3667. 
Bansil, A., R. Pankaluoto, R. S. Rao, P. E. Mijnarends, W. Długosz, R. Prasad, and L. C. Smedskjaer, 1988, Phys. Rev. Lett. 61, 2480. Barbiellini, B., S. Dugdale, and T. Jarlborg, 2003, Comput. Mater. Sci. 28, 287.

Barbiellini, B., M. Hakala, R. M. Nieminen, and M. J. Puska, 1999, MRS Proceedings 579, 249.

Barbiellini, B., M. Hakala, M. J. Puska, R. M. Nieminen, and A. A. Manuel, 1997, Phys. Rev. B 56, 7136.

Barbiellini, B., and P. M. Platzman, 2009, Phys. Status Solidi (c) 6, 2523.

Barbiellini, B., M. J. Puska, T. Korhonen, A. Harju, T. Torsti, and R. M. Nieminen, 1996, Phys. Rev. B 53, 16201.

Barbiellini, B., M. J. Puska, T. Torsti, and R. M. Nieminen, 1995, Phys. Rev. B 51, 7341.

Baruah, T., R. R. Zope, and A. Kshirsagar, 1999, Phys. Rev. B 60, 10770.

Bauer, G. E. W., 1983, Phys. Rev. B 27, 5912.

Becke, A. D., 1993, J. Chem. Phys. 98, 1372.

Becvar, F., J. Cizek, L. Lestak, I. Novotny, I. Prochazka, and F. Sebesta, 2000, Nucl. Instrum. Methods Phys. Res., Sect. A 443, 557.

Becvar, F., J. Cizek, and I. Prochazka, 2008, Appl. Surf. Sci. 255, 111.

Bergersen, B., and E. Pajanne, 1969, Phys. Rev. 186, 375.

Bergersen, B., E. Pajanne, P. Kubica, M. Stott, and C. Hodges, 1974, Solid State Commun. 15, 1377.

Bergersen, B., and M. Stott, 1969, Solid State Commun. 7, 1203.

Bergman, K., M. Stavola, S. J. Pearton, and J. Lopata, 1988, Phys. Rev. B 37, 2770.

Beringer, R., and C. G. Montgomery, 1942, Phys. Rev. 61, 222.

Berko, S., M. Haghgooie, and J. Mader, 1977, Phys. Lett. 63A, 335.

Berko, S., and F. L. Hereford, 1956, Rev. Mod. Phys. 28, 299.

Berko, S., and J. S. Plaskett, 1958, Phys. Rev. 112, 1877.

Bernardini, F., and V. Fiorentini, 1998, Phys. Rev. B 57, R9427.

Bertolaccini, L., and S. Zappa, 1967, Nuovo Cimento B 52, 487.

Bliss, D. E., W. Walukiewicz, J.W. Ager, E.E. Haller, K. T. Chan, and S. Tanigawa, 1992, J. Appl. Phys. 71, 1699.

Blöchl, P.E., 1990, Phys. Rev. B 41, 5414.

Blöchl, P.E., 1994, Phys. Rev. B 50, 17953.

Boev, O. V., M. J. Puska, and R. M. Nieminen, 1987, Phys. Rev. B 36, 7786 .

Bondarenko, V., J. Gebauer, F. Redmann, and R. Krause-Rehberg, 2005, Appl. Phys. Lett. 87, 161906.

Boninelli, S., A. Claverie, G. Impellizzeri, S. Mirabella, F. Priolo, E. Napolitani, and F. Cristiano, 2006, Appl. Phys. Lett. 89, 171916.

Boninelli, S., G. Impellizzeri, S. Mirabella, F. Priolo, E. Napolitani, N. Cherkashin, and F. Cristiano, 2008, Appl. Phys. Lett. 93, 061906.

Boroński, E., and R. M. Nieminen, 1986, Phys. Rev. B 34, 3820.

Børseth, T. M., F. Tuomisto, J. S. Christensen, E. V. Monakhov, B. G. Svensson, and A. Y. Kuznetsov, 2008, Phys. Rev. B 77, 045204.

Børseth, T. M., F. Tuomisto, J. S. Christensen, W. Skorupa, E. V. Monakhov, B. G. Svensson, and A. Y. Kuznetsov, 2006, Phys. Rev. B 74, 161202.

Botsoa, J., T. Sauvage, M.-P. Adam, P. Desgardin, E. Leoni, B. Courtois, F. Treussart, and M.-F. Barthe, 2011, Phys. Rev. B 84, 125209.

Bracht, H., J. F. Pedersen, N. Zangenberg, A. N. Larsen, E. E. Haller, G. Lulli, and M. Posselt, 2003, Phys. Rev. Lett. 91, 245502.

Brandt, W., S. Berko, and W.W. Walker, 1960, Phys. Rev. 120, 1289.

Brandt, W., and R. Paulin, 1977, Phys. Rev. B 15, 2511.

Brandt, W., and J. Reinheimer, 1970, Phys. Rev. B 2, 3104.

Brandt, W., and J. Reinheimer, 1971, Phys. Lett. 35A, 107.
Brauer, G., W. Anwand, P. G. Coleman, A. P. Knights, F. Plazaola, Y. Pacaud, W. Skorupa, J. Störmer, and P. Willutzki, 1996, Phys. Rev. B 54, 3084.

Brauer, G., J. Kuriplach, J. Cizek, W. Anwand, O. Melikhova, I. Prochazka, and W. Skorupa, 2007, Vacuum 81, 1314.

Bretagnon, T., S. Dannefaer, and D. Kerr, 1997, J. Appl. Phys. 81, 3446.

Campillo Robles, J. M., E. Ogando, and F. Plazaola, 2007, J. Phys. Condens. Matter 19, 176222.

Car, R., and M. Parrinello, 1985, Phys. Rev. Lett. 55, 2471.

Carbotte, J. P., 1966, Phys. Rev. 144, 309.

Carbotte, J. P., and S. Kahana, 1965, Phys. Rev. 139, A213.

Carvalho, A., A. Alkauskas, A. Pasquarello, A. Tagantsev, and N. Setter, 2009, Physica (Amsterdam) 404B, 4797.

Cassidy, D. B., R. G. Greaves, S. H. M. Deng, N. Lopez-Valdez, V. Meligne, and A. P. Mills, 2009, AIP Conf. Proc. 1099, 866.

Cassidy, D. B., and A. P. Mills, 2007, Nature (London) 449, 195.

Ceeh, H., C. Hugenschmidt, K. Schreckenbach, S. A. Gärtner, P. G. Thirolf, F. Fleischer, and D. Schwalm, 2011, Phys. Rev. A 84, 062508.

Chadi, D. J., and K. J. Chang, 1988, Phys. Rev. Lett. 60, 2187.

Chakraborty, B., 1981, Phys. Rev. B 24, 7423.

Chakraborty, B., and R. W. Siegel, 1983, Phys. Rev. B 27, 4535.

Charlton, M., and J.W. Humberston, 2001, Positron Physics, Cambridge Monographs on Atomic, Molecular, and Chemical Physics, Vol. 11 (Cambridge University Press, Cambridge, England).

Chen, Z. Q., K. Betsuyaku, and A. Kawasuso, 2008, Phys. Rev. B 77, 113204.

Chen, Z. Q., A. Kawasuso, Y. Xu, H. Naramoto, X. L. Yuan, T. Sekiguchi, R. Suzuki, and T. Ohdaira, 2005a, Phys. Rev. B 71, 115213.

Chen, Z. Q., A. Kawasuso, Y. Xu, H. Naramoto, X. L. Yuan, T. Sekiguchi, R. Suzuki, and T. Ohdaira, 2005b, J. Appl. Phys. 97, 013528.

Chen, Z. Q., M. Maekawa, A. Kawasuso, R. Suzuki, and T. Ohdaira, 2005, Appl. Phys. Lett. 87, 091910.

Chen, Z. Q., M. Maekawa, S. Yamamoto, A. Kawasuso, X. L. Yuan, T. Sekiguchi, R. Suzuki, and T. Ohdaira, 2004, Phys. Rev. B 69, 035210

Chen, Z. Q., S. J. Wang, M. Maekawa, A. Kawasuso, H. Naramoto, X. L. Yuan, and T. Sekiguchi, 2007, Phys. Rev. B 75, 245206.

Cheng, L. J., and C. K. Yeh, 1973, Solid State Commun. 12, 529.

Cheung, C. K., R. X. Wang, C. D. Beling, A. B. Djurii, and S. Fung, 2007, J. Phys. Condens. Matter 19, 086204.

Chichibu, S. F., K. Hazu, T. Onuma, and A. Uedono, 2011, Appl. Phys. Lett. 99, 051902.

Chichibu, S. F., H. Miyake, Y. Ishikawa, M. Tashiro, T. Ohtomo, K. Furusawa, K. Hazu, K. Hiramatsu, and A. Uedono, 2013, J. Appl. Phys. 113, 213506.

Chichibu, S. F., et al., 2006, Nat. Mater. 5, 810.

Cho, Y. K., J. Y. Leem, C. Lee, S. K. Noh, R. Suzuki, T. Odaira, and T. Mikado, 1997, Mater. Sci. Forum 255-257, 701.

Chow, K. H., L.S. Vlasenko, P. Johannesen, C. Bozdog, G. D. Watkins, A. Usui, H. Sunakawa, C. Sasaoka, and M. Mizuta, 2004, Phys. Rev. B 69, 045207.

Christoph Mueller, D., E. Alonso, and W. Fichtner, 2003, Phys. Rev. B 68, 045208.

Coleman, P. G., 2000, Ed., Positron Beams and Their Applications (World Scientific, Singapore).

Coleman, P. G., N.B. Chilton, and J.A. Baker, 1990, J. Phys. Condens. Matter 2, 9355.

Connors, D., and R. West, 1969, Phys. Lett. 30A, 24. 
Corbel, C., P. Moser, and M. Stucky, 1985, Ann. Chim. Sci. Mat. 10, 733.

Corbel, C., M. Stucky, P. Hautojärvi, K. Saarinen, and P. Moser, 1988, Phys. Rev. B 38, 8192.

Dabrowski, J., and M. Scheffler, 1988, Phys. Rev. Lett. 60, 2183.

Daniuk, S., 1983, Acta Phys. Pol. A 63, 633.

Daniuk, S., G. Kontrym-Sznajd, J. Majsnerowski, M. Šob, and H. Stachowiak, 1989, J. Phys. Condens. Matter 1, 6321.

Daniuk, S., G. Kontrym-Sznajd, A. Rubaszek, H. Stachowiak, J. Mayers, P. A. Walters, and R. N. West, 1987, J. Phys. F 17, 1365. Daniuk, S., M. Šob, and A. Rubaszek, 1991, Phys. Rev. B 43, 2580.

Dannefaer, S., 2009, J. Phys. Condens. Matter 21, 175412.

Dannefaer, S., B. Hogg, and D. Kerr, 1984, Phys. Rev. B 30, 3355.

Dannefaer, S., and K. Iakoubovskii, 2008, J. Phys. Condens. Matter 20, 235225.

Dannefaer, S., A. Pu, and D. Kerr, 2001, Diam. Relat. Mater. 10, 2113.

Dannefaer, S., W. Puff, and D. Kerr, 1997, Phys. Rev. B 55, 2182.

Dapor, M., 1996, J. Appl. Phys. 79, 8406.

Dekker, J., J. Oila, K. Saarinen, A. Tukiainen, W. Li, and M. Pessa, 2002, J. Appl. Phys. 92, 5942.

Delaney, P., B. Králik, and S. G. Louie, 1998, Phys. Rev. B 58, 4320.

Deng, A. H., P. Mascher, Y.W. Zhao, and L. Y. Lin, 2003, J. Appl. Phys. 93, 930.

Denison, A. B., and H.H. Farrell, 2004, Phys. Rev. B 69, 104302.

Desgardin, P., J. Oila, K. Saarinen, P. Hautojärvi, E. Tournié, J.-P.

Faurie, and C. Corbel, 2000, Phys. Rev. B 62, 15711.

Deutsch, M., 1951, Phys. Rev. 82, 455.

Diebel, M., and S. T. Dunham, 2004, Phys. Rev. Lett. 93, 245901.

Dirac, P. A. M., 1928, Proc. R. Soc. A 117, 610.

Dobaczewski, L., P. Kaczor, I. D. Hawkins, and A. R. Peaker, 1994, J. Appl. Phys. 76, 194.

Dong, A. W., C. Pascual-Izarra, S. J. Pas, A. J. Hill, B. J. Boyd, and C. J. Drummond, 2009, J. Phys. Chem. B 113, 84.

Downey, D. F., C. M. Osburn, and S. D. Marcus, 1997, Solid State Technol. 40, 71.

Drabold, D. A., and S. Estreicher, 2007, Eds., Theory of Defects in Semiconductors, Topics in Applied Physics, Vol. 104 (SpringerVerlag, Berlin).

Drummond, N. D., P. López Ríos, R. J. Needs, and C. J. Pickard, 2011, Phys. Rev. Lett. 107, 207402.

Drummond, N. D., P. López Ríos, C. J. Pickard, and R. J. Needs, 2010, Phys. Rev. B 82, 035107.

Duan, X. M., and C. Stampfl, 2008, Phys. Rev. B 77, 115207.

Duan, X. M., and C. Stampfl, 2009a, Phys. Rev. B 79, 035207.

Duan, X. M., and C. Stampfl, 2009b, Phys. Rev. B 79, 174202.

Edwardson, C. J., P. G. Coleman, H. A.W.E. Mubarek, and A.S. Gandy, 2012, J. Appl. Phys. 111, 073510.

Eijt, S. W.H., A. van Veen, H. Schut, P.E. Mijnarends, A. B. Denison, B. Barbiellini, and A. Bansil, 2006, Nat. Mater. 5, 23.

Eldrup, M., D. Lightbody, and J. Sherwood, 1981, Chem. Phys. 63, 51.

El Mubarek, H. A. W., and P. Ashburn, 2003, Appl. Phys. Lett. 83, 4134.

El Mubarek, H. A. W., P. Wang, R. Price, J. M. Bonar, J. Zhang, P. L.F. Hemment, and P. Ashburn, 2005, Mater. Sci. Semicond. Process. 8, 103.

Elsayed, M., V. Bondarenko, K. Petters, J. Gebauer, and R. KrauseRehberg, 2008, J. Appl. Phys. 104, 103526.

Elsayed, M., R. Krause-Rehberg, W. Anwand, M. Butterling, and B. Korff, 2011, Phys. Rev. B 84, 195208.

Ertekin, E., M.T. Winkler, D. Recht, A. J. Said, M. J. Aziz, T. Buonassisi, and J.C. Grossman, 2012, Phys. Rev. Lett. 108, 026401.
Fahey, P. M., P.B. Griffin, and J.D. Plummer, 1989, Rev. Mod. Phys. 61, 289.

Ferragut, R., A. Calloni, A. Dupasquier, and G. Isella, 2010, Nanoscale Res. Lett. 5, 1942.

Ferrell, R. A., 1956, Rev. Mod. Phys. 28, 308.

Fiorentini, V., F. Bernardini, F. Della Sala, A. Di Carlo, and P. Lugli, 1999, Phys. Rev. B 60, 8849.

Fleischer, S., C. D. Beling, S. Fung, W. R. Nieveen, J.E. Squire, J. Q. Zheng, and M. Missous, 1997, J. Appl. Phys. 81, 190.

Freysoldt, C., J. Neugebauer, and C. G. Van de Walle, 2009, Phys. Rev. Lett. 102, 016402.

Fujinami, M., 1996, Phys. Rev. B 53, 13047.

Fujiwara, K., T. Hyodo, and J. Ohyama, 1972, J. Phys. Soc. Jpn. 33, 1047.

Fukaya, Y., I. Mochizuki, and A. Kawasuso, 2012, Phys. Rev. B 86, 035423.

Gebauer, J., R. Krause-Rehberg, S. Eichler, M. Luysberg, H. Sohn, and E. R. Weber, 1997, Appl. Phys. Lett. 71, 638.

Gebauer, J., R. Krause-Rehberg, M. Prokesch, and K. Irmscher, 2002, Phys. Rev. B 66, 115206.

Gebauer, J., M. Lausmann, T.E. M. Staab, R. Krause-Rehberg, M. Hakala, and M. J. Puska, 1999, Phys. Rev. B 60, 1464.

Gentils, A., et al., 2010, Phys. Rev. B 81, 144109.

Ghosh, V. J., and G. C. Aers, 1995, Phys. Rev. B 51, 45.

Ghosh, V. J., M. Alatalo, P. Asoka-Kumar, B. Nielsen, K. G. Lynn, A.C. Kruseman, and P.E. Mijnarends, 2000, Phys. Rev. B 61, 10092.

Gilgien, L., G. Galli, F. Gygi, and R. Car, 1994, Phys. Rev. Lett. 72, 3214.

Gonze, X., et al., 2009, Comput. Phys. Commun. 180, 2582.

Gotz, W., N. M. Johnson, D. P. Bour, M. D. McCluskey, and E.E. Haller, 1996, Appl. Phys. Lett. 69, 3725.

Gregory, R. B., and Y. Zhu, 1990, Nucl. Instrum. Methods Phys. Res., Sect. A 290, 172.

Greif, H., M. Haaks, U. Holzwarth, U. Männig, M. Tongbhoyai, T. Wider, K. Maier, J. Bihr, and B. Huber, 1997, Appl. Phys. Lett. 71, 2115.

Guagliardo, P. R., E.R. Vance, Z. Zhang, J. Davis, J.F. Williams, and S. N. Samarin, 2012, J. Am. Ceram. Soc. 95, 1727.

Gullikson, E. M., and A. P. Mills, 1986, Phys. Rev. Lett. 57, 376.

Gunnarsson, O., M. Jonson, and B. I. Lundqvist, 1979, Phys. Rev. B 20, 3136.

Gupta, R. P., and R. W. Siegel, 1977, Phys. Rev. Lett. 39, 1212.

Gupta, R. P., and R. W. Siegel, 1980a, Phys. Rev. B 22, 4572.

Gupta, R. P., and R. W. Siegel, 1980b, J. Phys. F 10, L7.

Hakala, M., M. J. Puska, and R. M. Nieminen, 1998, Phys. Rev. B 57, 7621.

Hamann, D. R., M. Schlüter, and C. Chiang, 1979, Phys. Rev. Lett. 43, 1494.

Hanssen, K.E. H. M., and P.E. Mijnarends, 1986, Phys. Rev. B 34, 5009.

Hautakangas, S., I. Makkonen, V. Ranki, M. J. Puska, K. Saarinen, X. Xu, and D. C. Look, 2006, Phys. Rev. B 73, 193301.

Hautakangas, S., J. Oila, M. Alatalo, K. Saarinen, L. Liszkay, D. Seghier, and H. P. Gislason, 2003, Phys. Rev. Lett. 90, 137402.

Hautojärvi, P., 1979, Positron in Solids, Topics in Current Physics (Springer, New York).

Hautojärvi, P., J. Heiniö, M. Manninen, and R. Nieminen, 1977, Philos. Mag. 35, 973.

Hawari, A. I., D. W. Gidley, J. Xu, J. Moxom, A. G. Hathaway, B. Brown, and R. Vallery, 2009, AIP Conf. Proc. 1099, 862.

Hede, B., and J. Carbotte, 1972, J. Phys. Chem. Solids 33, 727.

Heiskanen, M., T. Torsti, M. J. Puska, and R. M. Nieminen, 2001, Phys. Rev. B 63, 245106. 
Henry, L., M.-F. Barthe, C. Corbel, P. Desgardin, G. Blondiaux, S. Arpiainen, and L. Liszkay, 2003, Phys. Rev. B 67, 115210.

Hetényi, B., F. D. Angelis, P. Giannozzi, and R. Car, 2001, J. Chem. Phys. 115, 5791.

Heyd, J., G. E. Scuseria, and M. Ernzerhof, 2003, J. Chem. Phys. 118, 8207.

Hickey, D. P., Z. L. Bryan, K. S. Jones, R. G. Elliman, and E. E. Haller, 2007, Appl. Phys. Lett. 90, 132114.

Hirata, K., Y. Kobayashi, and Y. Ujihira, 1996, J. Chem. Soc., Faraday Trans. 92, 985.

Hodges, C. H., 1970, Phys. Rev. Lett. 25, 284.

Hoffmann, L., A. Shukla, M. Peter, B. Barbiellini, and A. Manuel, 1993, Nucl. Instrum. Methods Phys. Res., Sect. A 335, 276.

Hohenberg, P., and W. Kohn, 1964, Phys. Rev. 136, B864.

Hubbard, J., 1969, J. Phys. C 2, 1222.

Hubbard, J., and P.E. Mijnarends, 1972, J. Phys. C 5, 2323.

Hugenschmidt, C., G. Kögel, R. Repper, K. Schreckenbach, P. Sperr, B. Strasser, and W. Triftshäuser, 2004, Nucl. Instrum. Methods Phys. Res., Sect. B 221, 160.

Hugenschmidt, C., J. Mayer, and K. Schreckenbach, 2010, J. Phys. Conf. Ser. 225, 012015.

Huomo, H., A. Vehanen, M. D. Bentzon, and P. Hautojärvi, 1987, Phys. Rev. B 35, 8252.

Hurle, D., 1979, J. Phys. Chem. Solids 40, 613.

Hyodo, T., T. McMullen, and A. T. Stewart, 1986, Phys. Rev. B 33, 3050.

Iakoubovskii, K., S. Dannefaer, and A. Stesmans, 2005, Phys. Rev. B 71, 233201.

Ichimiya, A., 1992, Solid State Phenom. 28-29, 143.

Ishibashi, S., 2004, Mater. Sci. Forum 445-446, 401.

Ishibashi, S., and M. Kohyama, 2003, Phys. Rev. B 67, 113403.

Ishibashi, S., A. A. Manuel, L. Hoffmann, and K. Bechgaard, 1997, Phys. Rev. B 55, 2048.

Ishibashi, S., A. A. Manuel, M. Kohyama, M. Tokumoto, and H. Anzai, 1999, Phys. Rev. B 60, R3747.

Ishibashi, S., T. Tamura, S. Tanaka, M. Kohyama, and K. Terakura, 2007, Phys. Rev. B 76, 153310.

Islam, M. M., A. Uedono, S. Ishibashi, K. Tenjinbayashi, T. Sakurai, A. Yamada, S. Ishizuka, K. Matsubara, S. Niki, and K. Akimoto, 2011, Appl. Phys. Lett. 98, 112105.

Janotti, A., J. L. Lyons, and C. G. Van de Walle, 2012, Phys. Status Solidi (a) 209, 65.

Janotti, A., and C.G. Van de Walle, 2009, Rep. Prog. Phys. 72, 126501.

Jarlborg, T., and A. K. Singh, 1987, Phys. Rev. B 36, 4660.

Jean, Y., 1990, Microchem. J. 42, 72.

Jean, Y.C., P. E. Mallon, and D. M. Schrader, 2003, Principles and Applications of Positron and Positronium Chemistry (World Scientific, Singapore).

Jensen, K. O., 1989, J. Phys. Condens. Matter 1, 10595.

Jensen, K. O., and A. B. Walker, 1988, J. Phys. F 18, L277.

Jensen, K. O., and A. B. Walker, 1990, J. Phys. Condens. Matter 2, 9757.

Jensen, K. O., and A. B. Walker, 1992, J. Phys. Condens. Matter 4, 1973.

Jensen, K. O., and A. Weiss, 1990, Phys. Rev. B 41, 3928.

Johansen, K. M., A. Zubiaga, I. Makkonen, F. Tuomisto, P.T. Neuvonen, K. E. Knutsen, E. V. Monakhov, A. Y. Kuznetsov, and B. G. Svensson, 2011, Phys. Rev. B 83, 245208.

Johansen, K. M., A. Zubiaga, F. Tuomisto, E. V. Monakhov, A. Y. Kuznetsov, and B. G. Svensson, 2011, Phys. Rev. B 84, 115203.

Jorch, H. H., K. G. Lynn, and T. McMullen, 1984, Phys. Rev. B 30, 93.

Jorgensen, L. V., A. C. Kruseman, H. Schut, A. VanVeen, M. Fanciulli, and T.D. Moustakas, 1997, in Materials Research
Society Symposia Proceedings, Vol. 449, edited by F. A. Ponce, T. D. Moustakas, I. Akasaki, and B. A. Monemar (North-Holland, New York), pp. 853-858.

Jung, W.-S., J.-H. Park, A. Nainani, D. Nam, and K. C. Saraswat, 2012, Appl. Phys. Lett. 101, 072104.

Kahana, S., 1960, Phys. Rev. 117, 123.

Kahana, S., 1963, Phys. Rev. 129, 1622.

Kaminska, M., and E.R. Weber, 1993, in Semiconductors and Semimetals, Vol. 38, edited by E. R. Weber (Academic, New York), p. 59.

Kaufmann, U., M. Kunzer, H. Obloh, M. Maier, C. Manz, A. Ramakrishnan, and B. Santic, 1999, Phys. Rev. B 59, 5561.

Kauppinen, H., L. Baroux, K. Saarinen, C. Corbel, and P. Hautojärvi, 1997, J. Phys. Condens. Matter 9, 5495.

Kauppinen, H., C. Corbel, J. Nissila, K. Saarinen, and P. Hautojärvi, 1998, Phys. Rev. B 57, 12911.

Kawasuso, A., Y. Fukaya, K. Hayashi, M. Maekawa, S. Okada, and A. Ichimiya, 2003, Phys. Rev. B 68, 241313.

Kawasuso, A., H. Itoh, S. Okada, and H. Okumura, 1996, J. Appl. Phys. 80, 5639.

Kawasuso, A., M. Maekawa, Y. Fukaya, A. Yabuuchi, and I. Mochizuki, 2011, Phys. Rev. B 83, 100406.

Kawasuso, A., M. Maekawa, Y. Fukaya, A. Yabuuchi, and I. Mochizuki, 2012, Phys. Rev. B 85, 024417.

Kawasuso, A., and S. Okada, 1998, Phys. Rev. Lett. 81, 2695.

Keeble, D. J., J. D. Major, L. Ravelli, W. Egger, and K. Durose, 2011, Phys. Rev. B 84, 174122.

Keeble, D. J., S. Singh, R. A. Mackie, M. Morozov, S. McGuire, and D. Damjanovic, 2007, Phys. Rev. B 76, 144109.

Keeble, D. J., M. T. Umlor, P. Asoka-Kumar, K. G. Lynn, and P. W. Cooke, 1993, Appl. Phys. Lett. 63, 87.

Kerker, G. P., 1980, J. Phys. C 13, L189.

Kilanski, L., A. Zubiaga, F. Tuomisto, W. Dobrowolski, V. Domukhovski, S.A. Varnavskiy, and S.F. Marenkin, 2009, J. Appl. Phys. 106, 013524.

Kilpeläinen, S., K. Kuitunen, F. Tuomisto, J. Slotte, H. H. Radamson, and A. Y. Kuznetsov, 2010, Phys. Rev. B 81, 132103.

Kilpeläinen, S., F. Tuomisto, J. Slotte, J. L. Hansen, and A. N. Larsen, 2011, Phys. Rev. B 83, 094115.

Kimball, G. E., and G. H. Shortley, 1934, Phys. Rev. 45, 815.

Knoll, G. F., 2000, Radiation Detection and Measurement (Wiley, New York).

Knutsen, K. E., A. Galeckas, A. Zubiaga, F. Tuomisto, G. C. Farlow, B. G. Svensson, and A. Y. Kuznetsov, 2012, Phys. Rev. B 86, 121203.

Kohn, W., and N. Rostoker, 1954, Phys. Rev. 94, 111.

Kohn, W., and L. J. Sham, 1965, Phys. Rev. 140, A1133.

Kontrym-Sznajd, G., 2009, Low Temp. Phys. 35, 599.

Kontrym-Sznajd, G., H. Sormann, and E. Boroński, 2012, Phys. Rev. B 85, 245104.

Korhonen, E., K. Kuitunen, F. Tuomisto, A. Urbaniak, M. Igalson, J. Larsen, L. Gütay, S. Siebentritt, and Y. Tomm, 2012, Phys. Rev. B 86, 064102.

Korhonen, T., M. J. Puska, and R. M. Nieminen, 1996, Phys. Rev. B 54, 15016.

Korringa, J., 1947, Physica (Utrecht) 13, 392.

Krause, R., A. Klimakow, F. Kiessling, A. Polity, P. Gille, and M. Schenk, 1990, J. Cryst. Growth 101, 512.

Krause, R., K. Saarinen, P. Hautojärvi, A. Polity, G. Gärtner, and C. Corbel, 1990, Phys. Rev. Lett. 65, 3329.

Krause-Rehberg, R., F. Börner, F. Redmann, J. Gebauer, R. Kögler, R. Kliemann, W. Skorupa, W. Egger, G. Kögel, and W. Triftshäuser, 2001, Physica B (Amsterdam) 308-310, 442. 
Krause-Rehberg, R., M. Brohl, H. S. Leipner, T. Drost, A. Polity, U. Beyer, and H. Alexander, 1993, Phys. Rev. B 47, 13266.

Krause-Rehberg, R., H. Leipner, T. Abgarjan, and A. Polity, 1998, Appl. Phys. A 66, 599.

Krause-Rehberg, R., and H. S. Leipner, 1999, Positron Annihilation in Semiconductors (Springer-Verlag, Berlin).

Krause-Rehberg, R., et al., 2011, J. Phys. Conf. Ser. 262, 012003. Kresse, G., and J. Furthmüller, 1996a, Comput. Mater. Sci. 6, 15. Kresse, G., and J. Furthmüller, 1996b, Phys. Rev. B 54, 11169.

Kresse, G., and J. Hafner, 1993, Phys. Rev. B 47, 558.

Kresse, G., and D. Joubert, 1999, Phys. Rev. B 59, 1758.

Kubica, P., and M. J. Stott, 1974, J. Phys. F 4, 1969.

Kubo, Y., S. Wakoh, and J. Yamashita, 1976, J. Phys. Soc. Jpn. 41, 830.

Kuisma, S., K. Saarinen, P. Hautojärvi, C. Corbel, and C. LeBerre, 1996, Phys. Rev. B 53, 9814.

Kuitunen, K., K. Saarinen, and F. Tuomisto, 2007, Phys. Rev. B 75, 045210 .

Kuitunen, K., F. Tuomisto, and J. Slotte, 2007, Phys. Rev. B 76, 233202.

Kuitunen, K., F. Tuomisto, J. Slotte, and I. Capan, 2008, Phys. Rev. B 78, 033202.

Kuriplach, J., G. Brauer, W. Anwand, and W. Skorupa, 2003, Phys. Rev. Lett. 91, 199601.

Laasonen, K., M. Alatalo, M. J. Puska, and R. M. Nieminen, 1991, J. Phys. Condens. Matter 3, 7217.

Laine, T., K. Saarinen, P. Hautojärvi, C. Corbel, and M. Missous, 1999, J. Appl. Phys. 86, 1888.

Laine, T., K. Saarinen, J. Mäkinen, P. Hautojärvi, C. Corbel, L. N. Pfeiffer, and P. H. Citrin, 1996, Phys. Rev. B 54, R11050.

Lam, L., and P. M. Platzman, 1974, Phys. Rev. B 9, 5122.

Lannoo, M., and J. Bourgoin, 1981, Point Defects in Semiconductors 1, Springer Series in Solid-State Sciences (Springer-Verlag, Berlin).

Lantto, L. J., 1987, Phys. Rev. B 36, 5160.

Lany, S., and A. Zunger, 2010, Appl. Phys. Lett. 96, 142114.

Laverock, J., T. D. Haynes, M. A. Alam, and S. B. Dugdale, 2010, Phys. Rev. B 82, 125127.

Lawther, D. W., U. Myler, P. J. Simpson, P. M. Rousseau, P. B. Griffin, and J. D. Plummer, 1995, Appl. Phys. Lett. 67, 3575.

Le Berre, C., C. Corbel, M. R. Brozel, S. Kuisma, K. Saarinen, and P. Hautojärvi, 1994, J. Phys. Condens. Matter 6, L759.

Le Berre, C., C. Corbel, K. Saarinen, S. Kuisma, P. Hautojärvi, and R. Fornari, 1995, Phys. Rev. B 52, 8112.

Lee, K., B. VanMil, M. Luo, T. H. Myers, A. Armstrong, S. A. Ringel, M. Rummukainen, and K. Saarinen, 2006, MRS Symp. Proc. 892, 729.

Lefebvre, P., A. Morel, M. Gallart, T. Taliercio, J. Allègre, B. Gil, H. Mathieu, B. Damilano, N. Grandjean, and J. Massies, 2001, Appl. Phys. Lett. 78, 1252.

Lennard, W. N., G. R. Massoumi, P. J. Schultz, P. J. Simpson, and G. C. Aers, 1995, Phys. Rev. Lett. 74, 3947.

Limpijumnong, S., and C. G. Van de Walle, 2004, Phys. Rev. B 69, 035207.

Ling, C. C., C. D. Beling, and S. Fung, 2000, Phys. Rev. B 62, 8016.

Liszkay, L., C. Corbel, L. Baroux, P. Hautojärvi, M. Bayhan, A. W. Brinkman, and S. Tatarenko, 1994, Appl. Phys. Lett. 64, 1380.

Look, D. C., J. W. Hemsky, and J. R. Sizelove, 1999, Phys. Rev. Lett. 82, 2552.

Lopez, G. M., and V. Fiorentini, 2006, Appl. Phys. Lett. 89, 092113.

Lopez, G. M., V. Fiorentini, G. Impellizzeri, S. Mirabella, and E. Napolitani, 2005, Phys. Rev. B 72, 045219.

Loucks, T. L., 1966, Phys. Rev. 144, 504.

Lynn, K. G., and B. Nielsen, 1987, Phys. Rev. Lett. 58, 81.
Ma, T. P., 1992, J. Vac. Sci. Technol. A 10, 705.

MacKenzie, I. K., T. L. Khoo, A. B. McDonald, and B. T. A. McKee, 1967, Phys. Rev. Lett. 19, 946.

Mackie, R. A., S. Singh, J. Laverock, S. B. Dugdale, and D. J. Keeble, 2009, Phys. Rev. B 79, 014102.

Mahony, J., P. Mascher, and W. Puff, 1996, J. Appl. Phys. 80, 2712.

Makhov, D. V., and L. J. Lewis, 2005, Phys. Rev. B 71, 205215.

Mäki, J.-M., T. Kuittinen, E. Korhonen, and F. Tuomisto, 2012, New J. Phys. 14, 035023.

Mäki, J.-M., I. Makkonen, F. Tuomisto, A. Karjalainen, S. Suihkonen, J. Räisänen, T. Y. Chemekova, and Y. N. Makarov, 2011, Phys. Rev. B 84, 081204.

Mäki, J.-M., F. Tuomisto, C. J. Kelly, D. Fisher, and P. M. Martineau, 2009, J. Phys. Condens. Matter 21, 364216.

Mäki, J.-M., F. Tuomisto, A. Varpula, D. Fisher, R. U. A. Khan, and P. M. Martineau, 2011, Phys. Rev. Lett. 107, 217403.

Mäkinen, J., C. Corbel, P. Hautojärvi, and D. Mathiot, 1991, Phys. Rev. B 43, 12114.

Mäkinen, J., T. Laine, K. Saarinen, P. Hautojärvi, C. Corbel, V. M. Airaksinen, and P. Gibart, 1993, Phys. Rev. Lett. 71, 3154.

Mäkinen, S., H. Rajainmäki, and S. Linderoth, 1990, Phys. Rev. B 42, 11166.

Makkonen, I., M. Hakala, and M. J. Puska, 2005, J. Phys. Chem. Solids 66, 1128.

Makkonen, I., M. Hakala, and M. J. Puska, 2006, Phys. Rev. B 73, 035103.

Makkonen, I., and M. J. Puska, 2007, Phys. Rev. B 76, 054119.

Makkonen, I., A. Snicker, M. J. Puska, J.-M. Mäki, and F. Tuomisto, 2010, Phys. Rev. B 82, 041307.

Makov, G., and M. C. Payne, 1995, Phys. Rev. B 51, 4014.

Makov, G., R. Shah, and M.C. Payne, 1996, Phys. Rev. B 53, 15513.

Manninen, M., and R. Nieminen, 1981, Appl. Phys. A 26, 93.

Manninen, M., R. Nieminen, P. Hautojärvi, and J. Arponen, 1975, Phys. Rev. B 12, 4012.

Martin, G. M., and S. Makram-Ebeid, 1986, in Deep Centers in Semiconductors, edited by S. Pantelides, Chap. 6 (Gordon and Breach, New York).

Martin, R. M., 2004, Electronic Structure: Basic Theory and Practical Methods (Cambridge University Press, Cambridge, England).

Mathiot, D., and J. C. Pfister, 1983, Appl. Phys. Lett. 42, 1043.

Maurer, P. C., et al., 2012, Science 336, 1283.

McCluskey, M., and E. Haller, 2012, Dopants and Defects in Semiconductors (Taylor \& Francis, London).

McMullen, T., and M. J. Stott, 1986, Phys. Rev. B 34, 8985.

Meng, X.-T., A. Zecca, R. S. Brusa, and W. Puff, 1994, Phys. Rev. B 50, 2657.

Meyer, B., K. Hummler, C. Elsasser, and M. Fahnle, 1995, J. Phys. Condens. Matter 7, 9201.

Mijnarends, P., 1973, Physica (Amsterdam) 63, 235.

Mijnarends, P. E., and A. Bansil, 1990, J. Phys. Condens. Matter 2, 911.

Mijnarends, P. E., and A. Bansil, 1993, in Positron Spectroscopy of Solids, edited by A. Dupasquier and A.P. Mills (IOS, Amsterdam).

Mijnarends, P. E., A. C. Kruseman, A. van Veen, H. Schut, and A. Bansil, 1998, J. Phys. Condens. Matter 10, 10383.

Mijnarends, P. E., and L. P.L. M. Rabou, 1986, J. Phys. F 16, 483.

Mijnarends, P.E., L.P.L.M. Rabou, K.E.H.M. Hanssen, and A. Bansil, 1987, Phys. Rev. Lett. 59, 720.

Mijnarends, P.E., and R. M. Singru, 1979, Phys. Rev. B 19, 6038. Mills, A. P., 1981, Phys. Rev. Lett. 46, 717.

Mills, A.P., and W.S. Crane, 1985, Phys. Rev. A 31, 593. 
Mills, A. P., and E. M. Gullikson, 1986, Appl. Phys. Lett. 49, 1121.

Mills, A.P., and P.M. Platzman, 2001, in New Directions in Antimatter Chemistry and Physics, edited by C.M. Surko, and F. A. Gianturco (Kluwer Academic Publishers, Dordrecht, The Netherlands), p. 115.

Mills, A. P., 1980, Appl. Phys. 22, 273.

Mitroy, J., and B. Barbiellini, 2002, Phys. Rev. B 65, 235103.

Mogensen, O.E., 1995, Positron Annihilation in Chemistry, Springer Series in Chemical Physics, Vol. 58 (Springer, Berlin/ Heidelberg).

Mohorovicic, S., 1934, Astron. Nachr. 253, 93.

Mooney, P. M. 1990, J. Appl. Phys. 67, R1.

Moses, P. G., M. Miao, Q. Yan, and C.G. Van de Walle, 2011, J. Chem. Phys. 134, 084703.

Moutanabbir, O., R. Scholz, U. Gösele, A. Guittoum, M. Jungmann, M. Butterling, R. Krause-Rehberg, W. Anwand, W. Egger, and P. Sperr, 2010, Phys. Rev. B 81, 115205.

Mukherjee, S., M. P. Nadesalingam, P. Guagliardo, A. D. Sergeant, B. Barbiellini, J.F. Williams, N.G. Fazleev, and A. H. Weiss, 2010, Phys. Rev. Lett. 104, 247403.

Myler, U., and P. J. Simpson, 1997, Phys. Rev. B 56, 14303.

Nagai, Y., M. Hasegawa, Z. Tang, A. Hempel, K. Yubuta, T. Shimamura, Y. Kawazoe, A. Kawai, and F. Kano, 2000, Phys. Rev. B 61, 6574.

Neugebauer, J., and C. G. Van de Walle, 1994, Phys. Rev. B 50, 8067.

Neugebauer, J., and C. G. Van de Walle, 1996, Appl. Phys. Lett. 69, 503.

Neuvonen, P. T., L. Vines, A. Y. Kuznetsov, B. G. Svensson, X. Du, F. Tuomisto, and A. Hallén, 2009, Appl. Phys. Lett. 95, 242111.

Neuvonen, P. T., L. Vines, V. Venkatachalapathy, A. Zubiaga, F. Tuomisto, A. Hallén, B. G. Svensson, and A. Y. Kuznetsov, 2011, Phys. Rev. B 84, 205202.

Nielsen, B., K. G. Lynn, and Y. C. Chen, 1986, Phys. Rev. Lett. 57, 1789.

Nieminen, R. M., 1975, J. Phys. C 8, 2077.

Nieminen, R. M., E. Boroński, and L. J. Lantto, 1985, Phys. Rev. B 32, 1377.

Niki, S., R. Suzuki, S. Ishibashi, T. Ohdaira, P. J. Fons, A. Yamada, H. Oyanagi, T. Wada, R. Kimura, and T. Nakada, 2001, Thin Solid Films 387, 129.

Nilen, R., S. Connell, D. Britton, C. Fischer, E. Sendezera, P. Schaaff, and J. Sellschop, 1997, Diam. Relat. Mater. 6, 1777.

Nissilä, J., M. Karppinen, K. Rytsölä, J. Oila, K. Saarinen, and P. Hautojärvi, 2001, Nucl. Instrum. Methods Phys. Res., Sect. A 466, 527.

Nissilä, J., K. Rytsölä, R. Aavikko, A. Laakso, K. Saarinen, and P. Hautojärvi, 2005, Nucl. Instrum. Methods Phys. Res., Sect. A 538, 778.

Nissilä, J., K. Saarinen, and P. Hautojärvi, 2001, Phys. Rev. B 63, 165202.

Nykänen, H., S. Suihkonen, L. Kilanski, M. Sopanen, and F. Tuomisto, 2012, Appl. Phys. Lett. 100, 122105.

Nylandsted Larsen, A., C. Christensen, and J. W. Petersen, 1999, J. Appl. Phys. 86, 4861.

Nylandsted Larsen, A., K. Kyllesbech Larsen, P.E. Andersen, and B. G. Svensson, 1993, J. Appl. Phys. 73, 691.

Oila, J., J. Kivioja, V. Ranki, K. Saarinen, D. C. Look, R. J. Molnar, S. S. Park, S. K. Lee, and J. Y. Han, 2003, Appl. Phys. Lett. 82, 3433.

Oila, J., K. Saarinen, T. Laine, P. Hautojärvi, P. Uusimaa, M. Pessa, and J. Likonen, 1999, Phys. Rev. B 59, R12 736.

Packan, P. A., 1999, Science 285, 2079.

Pandey, K.C., A. Erbil, G. S. Cargill, R.F. Boehme, and D. Vanderbilt, 1988, Phys. Rev. Lett. 61, 1282.
Pankratov, O., H. Huang, T. Diaz de la Rubia, and C. Mailhiot, 1997, Phys. Rev. B 56, 13172.

Park, R. M., M. B. Troffer, C. M. Rouleau, J. M. DePuydt, and M. A. Haase, 1990, Appl. Phys. Lett. 57, 2127.

Pask, J., B. Klein, P. Sterne, and C. Fong, 2001, Comput. Phys. Commun. 135, 1.

Pask, J.E., B. M. Klein, C. Y. Fong, and P. A. Sterne, 1999, Phys. Rev. B 59, 12352.

Peng, J.P., K. G. Lynn, M. T. Umlor, D. J. Keeble, and D. R. Harshman, 1994, Phys. Rev. B 50, 11247.

Pennycook, S. J., 2012, MRS Bull. 37, 943.

Perdew, J.P., M. Ernzerhof, and K. Burke, 1996, J. Chem. Phys. 105, 9982.

Pi, X. D., C. P. Burrows, and P. G. Coleman, 2003, Phys. Rev. Lett. 90, 155901.

Pinkney, H., D. A. Thompson, B. J. Robinson, P. Mascher, P. J. Simpson, U. Myler, J. U. Kang, and M. Y. Frankel, 1998, J. Vac. Sci. Technol. A 16, 772.

Plazaola, F., A.P. Seitsonen, and M. J. Puska, 1994, J. Phys. Condens. Matter 6, 8809.

Polity, A., S. Huth, and M. Lausmann, 1999, Phys. Rev. B 59, 10603.

Polity, A., and F. Rudolf, 1999, Phys. Rev. B 59, 10025.

Probert, M. I. J., and M. C. Payne, 2003, Phys. Rev. B 67, 075204.

$\mathrm{Pu}, \mathrm{A}$., T. Bretagnon, D. Kerr, and S. Dannefaer, 2000, Diam. Relat. Mater. 9, 1450.

Puff, W., S. Brunner, P. Mascher, and A. G. Balogh, 1995, Mater. Sci. Forum 196-201, 333.

Puska, M. J., 1991, J. Phys. Condens. Matter 3, 3455.

Puska, M. J., C. Corbel, and R. M. Nieminen, 1990, Phys. Rev. B 41, 9980.

Puska, M. J., O. Jepsen, O. Gunnarsson, and R. M. Nieminen, 1986, Phys. Rev. B 34, 2695.

Puska, M. J., P. Lanki, and R. M. Nieminen, 1989, J. Phys. Condens. Matter 1, 6081.

Puska, M. J., S. Mäkinen, M. Manninen, and R. M. Nieminen, 1989, Phys. Rev. B 39, 7666.

Puska, M. J., and M. Manninen, 1987, J. Phys. F 17, 2235.

Puska, M. J., and R. M. Nieminen, 1983, J. Phys. F 13, 333.

Puska, M. J., and R. M. Nieminen, 1994, Rev. Mod. Phys. 66, 841.

Puska, M. J., S. Pöykkö, M. Pesola, and R. M. Nieminen, 1998, Phys. Rev. B 58, 1318.

Puska, M. J., A. P. Seitsonen, and R. M. Nieminen, 1995, Phys. Rev. B 52, 10947.

Puska, M. J., M. Šob, G. Brauer, and T. Korhonen, 1994, Phys. Rev. B 49, 10947.

Quinn, J. F., S. J. Pas, A. Quinn, H. P. Yap, R. Suzuki, F. Tuomisto, B. S. Shekibi, J. I. Mardel, A. J. Hill, and F. Caruso, 2012, J. Am. Chem. Soc. 134, 19808.

Ramamoorthy, M., and S. T. Pantelides, 1996, Phys. Rev. Lett. 76, 4753.

Ranki, V., J. Nissilä, and K. Saarinen, 2002, Phys. Rev. Lett. 88, 105506.

Ranki, V., A. Pelli, and K. Saarinen, 2004, Phys. Rev. B 69, 115205.

Ranki, V., and K. Saarinen, 2004, Phys. Rev. Lett. 93, 255502.

Rauch, C., I. Makkonen, and F. Tuomisto, 2011, Phys. Rev. B 84, 125201.

Rempel, A. A., W. Sprengel, K. Blaurock, K. J. Reichle, J. Major, and H.-E. Schaefer, 2002, Phys. Rev. Lett. 89, 185501.

Reshchikov, M.A., and H. Morkoç, 2005, J. Appl. Phys. 97, 061301.

Reurings, F., and A. Laakso, 2007, Phys. Status Solidi (c) 4, 3965.

Rinke, P., A. Schleife, E. Kioupakis, A. Janotti, C. Rödl, F. Bechstedt, M. Scheffler, and C.G. Van de Walle, 2012, Phys. Rev. Lett. 108, 126404. 
Rubaszek, A., 1991, Phys. Rev. B 44, 10857.

Rubaszek, A., Z. Szotek, and W. M. Temmerman, 1998, Phys. Rev. B 58, 11285.

Rubaszek, A., Z. Szotek, and W. M. Temmerman, 2000, Phys. Rev. B 61, 10100.

Rubaszek, A., Z. Szotek, and W. M. Temmerman, 2001, Phys. Rev. B 63, 165115.

Rubaszek, A., Z. Szotek, and W. M. Temmerman, 2002, Phys. Rev. B 65, 125104.

Rummukainen, M., I. Makkonen, V. Ranki, M. J. Puska, K. Saarinen, and H.-J.L. Gossmann, 2005, Phys. Rev. Lett. 94, 165501 .

Rummukainen, M., J. Oila, A. Laakso, K. Saarinen, A. J. Ptak, and T. H. Myers, 2004, Appl. Phys. Lett. 84, 4887.

Rummukainen, M., J. Slotte, K. Saarinen, H.H. Radamson, J. Hållstedt, and A. Y. Kuznetsov, 2006, Phys. Rev. B 73, 165209.

Rytsölä, K., J. Nissilä, J. Kokkonen, A. Laakso, R. Aavikko, and K. Saarinen, 2002, Appl. Surf. Sci. 194, 260.

Saarinen, K., 2000, in III-Nitride Semiconductors: Electrical, Structural and Defects Properties, edited by O. Manasreh (Elsevier, Amsterdam), pp. 109-149.

Saarinen, K., P. Hautojärvi, and C. Corbel, 1998, in Identification of Defects in Semiconductors, Semiconductors and Semimetals, Vol.51A, edited by M. Stavola (Academic Press, New York), p. 209.

Saarinen, K., P. Hautojärvi, P. Lanki, and C. Corbel, 1991, Phys. Rev. B 44, 10585.

Saarinen, K., S. Kuisma, P. Hautojärvi, C. Corbel, and C. LeBerre, 1994, Phys. Rev. B 49, 8005.

Saarinen, K., S. Kuisma, J. Mäkinen, P. Hautojärvi, M. Törnqvist, and C. Corbel, 1995, Phys. Rev. B 51, 14152.

Saarinen, K., et al., 1997, Phys. Rev. Lett. 79, 3030.

Saarinen, K., T. Laine, K. Skog, J. Mäkinen, P. Hautojärvi, K. Rakennus, P. Uusimaa, A. Salokatve, and M. Pessa, 1996, Phys. Rev. Lett. 77, 3407.

Saarinen, K., J. Nissilä, H. Kauppinen, M. Hakala, M. J. Puska, P. Hautojärvi, and C. Corbel, 1999, Phys. Rev. Lett. 82, 1883.

Saarinen, K., and V. Ranki, 2003, J. Phys. Condens. Matter 15, S2791.

Saito, H., Y. Nagashima, T. Kurihara, and T. Hyodo, 2002, Nucl. Instrum. Methods Phys. Res., Sect. A 487, 612.

Saito, M., and A. Oshiyama, 1996, Phys. Rev. B 53, 7810.

Saito, M., A. Oshiyama, and S. Tanigawa, 1991, Phys. Rev. B 44, 10601.

Salvadori, A., and J. P. Carbotte, 1969, Phys. Rev. 188, 550.

Sane, P., E. Salonen, E. Falck, J. Repakova, F. Tuomisto, J. M. Holopainen, and I. Vattulainen, 2009, J. Phys. Chem. B 113, 1810.

Saniz, R., B. Barbiellini, and A. Denison, 2002, Phys. Rev. B 65 , 245310.

Saniz, R., B. Barbiellini, P. M. Platzman, and A. J. Freeman, 2007, Phys. Rev. Lett. 99, 096101.

Saniz, R., B. Barbiellini, P. M. Platzman, and A. J. Freeman, 2008, Phys. Rev. Lett. 100, 019902.

Sann, J., A. Hofstaetter, D. Pfisterer, J. Stehr, and B. K. Meyer, 2006, Phys. Status Solidi (c) 3, 952.

Schödlbauer, D., G. Kögel, P. Sperr, and W. Triftshäuser, 1987, Phys. Status Solidi (a) 102, 549.

Schultz, P. A., 2000, Phys. Rev. Lett. 84, 1942.

Schultz, P. J., and K. G. Lynn, 1988, Rev. Mod. Phys. 60, 701.

Schut, H., A. van Veen, J. de Roode, and F. Labohm, 2004, Mater. Sci. Forum 445-446, 507.

Seitsonen, A. P., M. J. Puska, and R. M. Nieminen, 1995, Phys. Rev. B 51, 14057.

Selim, F. A., M. H. Weber, D. Solodovnikov, and K. G. Lynn, 2007, Phys. Rev. Lett. 99, 085502.
Shan, Y. Y., H. L. Au, C. C. Ling, T. C. Lee, B. K. Panda, S. Fung, C. D. Beling, Y. Y. Wang, and H. M. Weng, 1994, Appl. Phys. A 59, 259.

Shimizu, R., and D. Ze-Jun, 1992, Rep. Prog. Phys. 55, 487.

Shoukri, K. M., Y.M. Haddara, A. P. Knights, and P. G. Coleman, 2005, Appl. Phys. Lett. 86, 131923.

Shukla, A., M. Peter, and L. Hoffmann, 1993, Nucl. Instrum. Methods Phys. Res., Sect. A 335, 310.

Sihto, S.-L., J. Slotte, J. Lento, K. Saarinen, E. V. Monakhov, A. Y. Kuznetsov, and B. G. Svensson, 2003, Phys. Rev. B 68, 115307.

Simpson, P. J., Z. Jenei, P. Asoka-Kumar, R. R. Robison, and M.E. Law, 2004, Appl. Phys. Lett. 85, 1538.

Simpson, R. I., M. G. Stewart, C. D. Beling, and M. Charlton, 1990, J. Phys. Condens. Matter 2, 7255.

Singh, A. K., and T. Jarlborg, 1985, J. Phys. F 15, 727.

Singru, R. M., and P.E. Mijnarends, 1974, Phys. Rev. B 9, 2372.

Skriver, H. C., 1984, The LMTO Method (Springer, New York).

Slater, J. C., 1937, Phys. Rev. 51, 846.

Slotte, J., S. Kilpeläinen, F. Tuomisto, J. Räisänen, and A. Nylandsted Larsen, 2011, Phys. Rev. B 83, 235212.

Slotte, J., M. Rummukainen, F. Tuomisto, V.P. Markevich, A. R. Peaker, C. Jeynes, and R. M. Gwilliam, 2008, Phys. Rev. B 78, 085202.

Slotte, J., K. Saarinen, A. Salmi, S. Simula, R. Aavikko, and P. Hautojärvi, 2003, Phys. Rev. B 67, 115209.

Slotte, J., F. Tuomisto, K. Saarinen, C. G. Moe, S. Keller, and S. P. DenBaars, 2007, Appl. Phys. Lett. 90, 151908.

Smedskjaer, L. C., R. Benedek, R. W. Siegel, D. G. Legnini, M. D. Stahulak, and A. Bansil, 1987, Phys. Rev. Lett. 59, 2479.

Šob, M., 1978, in Proceedings of the 8th Annual International Symposium on Electronic Structure of Metals and Alloys, Gaussig, Germany, edited by P. Ziesche (Tech. Universität Dresden, Dresden), p. 170.

Šob, M., 1979, in Proceedings of the 5th International Conference on Positron Annihilation, Lake Yamanaka, Japan), edited by R. R. Hasiguti and K. Fujiwara (The Japanese Institute of Metals, Sendai), p. 309.

Šob, M., H. Sormann, and J. Kuriplach, 2003, in $D V-X \alpha$ for Advanced Nano Materials and other Interesting Topics in Materials Science, Advances in Quantum Chemistry, Vol. 42, edited by E. J. Brändas, H. Adachi, M. Uda, and R. Sekine (Academic Press, New York), pp. 77-108.

Soininen, E., A. Schwab, and K. G. Lynn, 1991, Phys. Rev. B 43, 10051.

Son, N. T., C. G. Hemmingsson, T. Paskova, K. R. Evans, A. Usui, N. Morishita, T. Ohshima, J. Isoya, B. Monemar, and E. Janzén, 2009, Phys. Rev. B 80, 153202.

Soven, P., 1967, Phys. Rev. 156, 809.

Soven, P., 1969, Phys. Rev. 178, 1136.

Stampfl, C., C. G. Van de Walle, D. Vogel, P. Krüger, and J. Pollmann, 2000, Phys. Rev. B 61, R7846.

Stavola, M., 1998, Ed., Identification of Defects in Semiconductors, Semiconductors and Semimetals, Vol. 51, Pt. A (Elsevier, New York).

Sterne, P., J. Pask, and B. Klein, 1999, Appl. Surf. Sci. 149, 238.

Sterne, P. A., and J. H. Kaiser, 1991, Phys. Rev. B 43, 13892.

Störmer, J., W. Triftshäuser, N. Hozhabri, and K. Alavi, 1996, Appl. Phys. Lett. 69, 1867.

Stott, M. J., and P. Kubica, 1975, Phys. Rev. B 11, 1.

Stott, M. J., and R. N. West, 1978, J. Phys. F 8, 635.

Sun, L., J. Chen, J. Li, and H. Jiang, 2010, Appl. Phys. Lett. 97, 191103.

Suzuki, R., Y. Kobayashi, T. Mikado, H. Ohgaki, M. Chiwaki, T. Yamazaki, A. Uedono, S. Tanigawa, and H. Funamoto, 1992, Jpn. J. Appl. Phys. 31, 2237. 
Suzuki, R., et al., 1997, Mater. Sci. Forum 255-257, 714.

Svensson, B. G., K. H. Ryden, and B.M.S. Lewerentz, 1989, J. Appl. Phys. 66, 1699.

Szpala, S., P. Asoka-Kumar, B. Nielsen, J. P. Peng, S. Hayakawa, K. G. Lynn, and H.-J. Gossmann, 1996, Phys. Rev. B 54, 4722.

Tang, Z., M. Hasegawa, T. Chiba, M. Saito, A. Kawasuso, Z. Q. Li, R. T. Fu, T. Akahane, Y. Kawazoe, and S. Yamaguchi, 1997, Phys. Rev. Lett. 78, 2236.

Tang, Z., M. Hasegawa, Y. Nagai, and M. Saito, 2002, Phys. Rev. B 65, 195108.

Tang, Z., M. Hasegawa, Y. Nagai, M. Saito, and Y. Kawazoe, 2002, Phys. Rev. B 65, 045108.

Tang, Z., Y. Nagai, K. Inoue, T. Toyama, T. Chiba, M. Saito, and M. Hasegawa, 2005, Phys. Rev. Lett. 94, 106402.

Tao, S. J., 1972, J. Chem. Phys. 56, 5499.

Tashiro, M., Y. Honda, T. Yamaguchi, P. Pujari, N. Kimura, T. Kozawa, G. Isoyama, and S. Tagawa, 2001, Radiat. Phys. Chem. 60, 529.

Toivonen, J., T. Hakkarainen, M. Sopanen, H. Lipsanen, J. Oila, and K. Saarinen, 2003, Appl. Phys. Lett. 82, 40.

Tong, B. Y., 1972, Phys. Rev. B 5, 1436.

Torsti, T., M. Heiskanen, M. J. Puska, and R. M. Nieminen, 2003, Int. J. Quantum Chem. 91, 171.

Torsti, T., et al., 2006, Phys. Status Solidi B 243, 1016.

Toyama, T., Z. Tang, K. Inoue, T. Chiba, T. Ohkubo, K. Hono, Y. Nagai, and M. Hasegawa, 2012, Phys. Rev. B 86, 104106.

Triftshäuser, W., G. Kögel, P. Sperr, D. Britton, K. Uhlmann, and P. Willutzki, 1997, Nucl. Instrum. Methods Phys. Res., Sect. B 130, 264.

Troullier, N., and J. L. Martins, 1991, Phys. Rev. B 43, 1993.

Tuomisto, F., 2010, in Technology of Gallium Nitride Crystal Growth, edited by D. Ehrentraut, E. Meissner, and M. Bockowski (Springer, Berlin/Heidelberg), pp. 295-316.

Tuomisto, F., and D. C. Look, 2007, Proc. SPIE Int. Soc. Opt. Eng. 6474, 647413.

Tuomisto, F., T. Paskova, R. Kröger, S. Figge, D. Hommel, B. Monemar, and R. Kersting, 2007, Appl. Phys. Lett. 90, 121915.

Tuomisto, F., A. Pelli, K. M. Yu, W. Walukiewicz, and W. J. Schaff, 2007, Phys. Rev. B 75, 193201.

Tuomisto, F., K. Pennanen, K. Saarinen, and J. Sadowski, 2004, Phys. Rev. Lett. 93, 055505.

Tuomisto, F., V. Ranki, D. C. Look, and G. C. Farlow, 2007, Phys. Rev. B 76, 165207.

Tuomisto, F., V. Ranki, K. Saarinen, and D. C. Look, 2003, Phys. Rev. Lett. 91, 205502.

Tuomisto, F., K. Saarinen, D.C. Look, and G.C. Farlow, 2005, Phys. Rev. B 72, 085206.

Tuomisto, F., K. Saarinen, B. Lucznik, I. Grzegory, H. Teisseyre, T. Suski, S. Porowski, P. R. Hageman, and J. Likonen, 2005, Appl. Phys. Lett. 86, 031915.

Uedono, A., S. Ishibashi, T. Ohdaira, and R. Suzuki, 2009, J. Cryst. Growth 311, 3075.

Uedono, A., S. Ishibashi, T. Watanabe, X. Q. Wang, S. T. Liu, G. Chen, L. W. Sang, M. Sumiya, and B. Shen, 2012, J. Appl. Phys. 112, 014507.

Uedono, A., K. Ito, H. Nakamori, K. Mori, Y. Nakano, T. Kachi, S. Ishibashi, T. Ohdaira, and R. Suzuki, 2007, J. Appl. Phys. 102, 084505 .

Uedono, A., T. Kitano, K. Hamada, T. Moriya, T. Kawano, S. Tanigawa, R. Suzuki, T. Ohdaira, and T. Mikado, 1997, Jpn. J. Appl. Phys. 36, 2571.
Uedono, A., K. Mori, N. Morishita, H. Itoh, S. Tanigawa, S. Fujii, and S. Shikata, 1999, J. Phys. Condens. Matter 11, 4925.

Uedono, A., K. Shimayama, M. Kiyohara, Z. Q. Chen, and K. Yamabe, 2002, J. Appl. Phys. 92, 2697.

Umeda, T., J. Isoya, T. Ohshima, S. Onoda, N. Morishita, K. Okonogi, and S. Shiratake, 2010, Appl. Phys. Lett. 97, 041911.

Valkealahti, S., and R. M. Nieminen, 1984, Appl. Phys. A 35, 51.

Vanderbilt, D., 1985, Phys. Rev. B 32, 8412.

Vanderbilt, D., 1990, Phys. Rev. B 41, 7892.

Van de Walle, C. G., J. L. Lyons, and A. Janotti, 2010, Phys. Status Solidi (a) 207, 1024.

Van de Walle, C. G., and J. Neugebauer, 2004, J. Appl. Phys. 95, 3851.

van Resandt, R.W.W., R.H. Vogel, and S.W. Provencher, 1982, Rev. Sci. Instrum. 53, 1392.

van Veen, A, H. Schut, J. de Vries, R. A. Hakvoort, and M. R. Ijpma, 1991, AIP Conf. Proc. 218, 171.

Velický, B., S. Kirkpatrick, and H. Ehrenreich, 1968, Phys. Rev. $175,747$.

Vollenweider, K., B. Sahli, and W. Fichtner, 2009, Phys. Rev. Lett. 103, 075503 .

Vollenweider, K., B. Sahli, and W. Fichtner, 2010, Phys. Rev. B 81, 174119.

Wahl, U., A. Vantomme, J. De Wachter, R. Moons, G. Langouche, J. G. Marques, and J. G. Correia (ISOLDE Collaboration), 1997, Phys. Rev. Lett. 79, 2069.

Wakoh, S., Y. Kubo, and J. Yamashita, 1975, J. Phys. Soc. Jpn. 38, 416.

Waldherr, G., J. Beck, P. Neumann, R. S. Said, M. Nitsche, M. L. Markham, D. J. Twitchen, J. Twamley, F. Jelezko, and J. Wrachtrup, 2012, Nat. Nanotechnol. 7, 105.

Wardle, M. G., J. P. Goss, and P. R. Briddon, 2005, Phys. Rev. B 71, 155205.

Watkins, G. D., 1986, in Deep Centers in Semiconductors, edited by S. Pantelides (Gordon and Breach, New York), p. 147.

Watkins, G. D., 2000, Mater. Sci. Semicond. Process. 3, 227.

Watkins, G. D., and J.W. Corbett, 1964, Phys. Rev. 134, A1359.

Watkins, G. D., and J.W. Corbett, 1965, Phys. Rev. 138, A543.

Weber, M. H., K. G. Lynn, B. Barbiellini, P. A. Sterne, and A. B. Denison, 2002, Phys. Rev. B 66, 041305.

Weiss, A., M. Jibaly, C. Lei, D. Mehl, R. Mayer, and K. G. Lynn, 1989, in Positron Annihilation, edited by L. DorikensVanpraet, M. Dorikens, and D. Segers (World Scientific, Singapore), p. 357.

West, R. N., 1973, Positron Studies of Condensed Matter, Advances in Physics (Taylor \& Francis, London).

West, R. N., 1993, in Positron Spectroscopy of Solids, edited by A. Dupasquier, and A.P. Mills (IOS, Amsterdam).

Wiktor, J., G. Jomard, M. Torrent, and M. Bertolus, 2013, Phys. Rev. B 87, 235207.

Williams, J. D., and P. Ashburn, 1992, J. Appl. Phys. 72, 3169.

Xie, J., and S. P. Chen, 1999, Phys. Rev. Lett. 83, 1795.

Yu, K. M., W. Walukiewicz, T. Wojtowicz, I. Kuryliszyn, X. Liu, Y. Sasaki, and J. K. Furdyna, 2002, Phys. Rev. B 65, 201303.

Zubiaga, A., F. Tuomisto, V. A. Coleman, H. H. Tan, C. Jagadish, K. Koike, S. Sasa, M. Inoue, and M. Yano, 2008, Phys. Rev. B 78, 035125 .

Zubiaga, A., F. Tuomisto, and M. J. Puska, 2012, Phys. Rev. A 85, 052707. 